\title{
Carbocation-Mediated Cyclization of Trienes in Acid Zeolites
}

Jason S. Lee ${ }^{\star_{1,2}}$, Stavros Caratzoulas 3 , Raul F. Lobo ${ }^{1,2,3}$

'Department of Chemical and Biomolecular Engineering, University of Delaware, Newark,

Delaware 19716

${ }^{2}$ Center for Neutron Research, Department of Chemical and Biomolecular Engineering, University of Delaware, Newark, Delaware 19716

${ }^{3}$ Catalysis Center for Energy Innovation, Department of Chemical and Biomolecular Engineering, University of Delaware, Newark, Delaware 19716

\section{SUPPORTING INFORMATION}

Table S1. DFT Energies for key intermediates in the Gas Phase: mo62x/6-31g(d,p)

\begin{tabular}{|l|l|l|l|l|l|}
\hline Intermediate or TS & Electronic Energy & $\begin{array}{l}\text { Zero-Point } \\
\text { Corrected }\end{array}$ & Energy (U) & Enthalpy (H) & Free Energy (G) \\
\hline R & -233.27934 & -233.15924 & -233.15257 & -233.15163 & -233.18948 \\
\hline A & -233.6102 & -233.47699 & -233.47055 & -233.4696 & -233.50684 \\
\hline B & -233.65993 & -233.52705 & -233.52087 & -233.51993 & -233.55672 \\
\hline C & -233.62923 & -233.49445 & -233.48897 & -233.48802 & -233.52292 \\
\hline F & -233.313008 & -233.191488 & -233.185736 & -233.184792 & -233.220355 \\
\hline J & -233.6193 & -233.4875 & -233.48024 & -233.4793 & -233.51846 \\
\hline K & -233.61361 & -233.48182 & -233.47491 & -233.47396 & -233.51219 \\
\hline L & -233.6417 & -233.50801 & -233.50206 & -233.50111 & -233.53737 \\
\hline M & -233.64256 & -233.50779 & -233.50224 & -233.5013 & -233.5364 \\
\hline P & -233.31101 & -233.18749 & -233.18233 & -233.18138 & -233.21572 \\
\hline
\end{tabular}

All reported quantities are in units of Hartrees. Thermal corrections for U, H, and G were calculated at $298 \mathrm{~K}$.

Table S2. DFT Energies for key intermediates in FAU: mo62x/6-31g(d,p)

\begin{tabular}{|l|l|l|l|l|l|}
\hline Intermediate or TS & Electronic Energy & $\begin{array}{l}\text { Zero-Point } \\
\text { Corrected }\end{array}$ & Energy (U) & Enthalpy (H) & Free Energy (G) \\
\hline Zeolite Cluster & -10649.53728 & -10649.27425 & -10649.21599 & -10649.21504 & -10649.35556 \\
\hline$R^{*}$ & -10882.84717 & -10882.46227 & -10882.39646 & -10882.39551 & -10882.55129 \\
\hline$A^{*}$ & -10882.84475 & -10882.45527 & -10882.39037 & -10882.38942 & -10882.54539 \\
\hline$B^{*}$ & -10882.87472 & -10882.48795 & -10882.42322 & -10882.42227 & -10882.57601 \\
\hline$C^{*}$ & -10882.87810 & -10882.48627 & -10882.42326 & -10882.42231 & -10882.57212 \\
\hline$F^{*}$ & -10882.88216 & -10882.4967 & -10882.43155 & -10882.4306 & -10882.58584 \\
\hline$G^{*}$ & -10882.87714 & -10882.48607 & -10882.42271 & -10882.42177 & -10882.5712 \\
\hline$J^{*}$ & -10882.85339 & -10882.46558 & -10882.40052 & -10882.39958 & -10882.55407 \\
\hline$K^{*}$ & -10882.84909 & -10882.46131 & -10882.39648 & -10882.39553 & -10882.54837 \\
\hline$L^{*}$ & -10882.8636 & -10882.47632 & -10882.41176 & -10882.41081 & -10882.56418 \\
\hline$M^{*}$ & -10882.86258 & -10882.47454 & -10882.41002 & -10882.40908 & -10882.56296 \\
\hline$P^{*}$ & -10882.88093 & -10882.49239 & -10882.42787 & -10882.42693 & -10882.58157 \\
\hline
\end{tabular}

All reported quantities are in units of Hartrees. Thermal corrections for U, H, and G were calculated at $298 \mathrm{~K}$. 
Table S3. DFT Energies for transition states in the Gas Phase: mo62x/6-31g(d,p)

\begin{tabular}{|l|l|l|l|l|l|}
\hline Intermediate or TS & Electronic Energy & $\begin{array}{l}\text { Zero-Point } \\
\text { Corrected }\end{array}$ & Energy (U) & Enthalpy (H) & Free Energy (G) \\
\hline$(\mathrm{A}-\mathrm{B}) \ddagger$ & -233.56601 & -233.43581 & -233.43006 & -233.42911 & -233.4647 \\
\hline$(\mathrm{B}-\mathrm{C}) \ddagger$ & -233.57161 & -233.44061 & -233.43494 & -233.43399 & -233.46994 \\
\hline$(\mathrm{B}-\mathrm{L}) \ddagger$ & -233.6035609 & -233.47404 & -233.468412 & -233.467468 & -233.502795 \\
\hline$(\mathrm{J}-\mathrm{K}) \ddagger$ & -233.59705 & -233.46703 & -233.46024 & -233.45929 & -233.49756 \\
\hline$(\mathrm{K}-\mathrm{L}) \ddagger$ & -233.60414 & -233.47272 & -233.46678 & -233.46584 & -233.50192 \\
\hline$(\mathrm{L}-\mathrm{M}) \ddagger$ & -233.59994 & -233.46712 & -233.46222 & -233.46127 & -233.49508 \\
\hline
\end{tabular}

All reported quantities are in units of Hartrees. Thermal corrections for U, H, and G were calculated at $298 \mathrm{~K}$.

Table S4. DFT Energies for transition states in FAU: mo62x/6-31g(d,p)

\begin{tabular}{|l|l|l|l|l|l|}
\hline Intermediate or TS & Electronic Energy & $\begin{array}{l}\text { Zero-Point } \\
\text { Corrected }\end{array}$ & Energy (U) & Enthalpy $(\mathrm{H})$ & Free Energy (G) \\
\hline$(\mathrm{R}-\mathrm{A}) \ddagger^{*}$ & -10882.81366 & -10882.43267 & -10882.36732 & -10882.36638 & -10882.52151 \\
\hline$(\mathrm{A}-\mathrm{B}) \ddagger^{*}$ & -10882.78029 & -10882.39629 & -10882.33175 & -10882.33081 & -10882.48454 \\
\hline$(\mathrm{B}-\mathrm{C}) \ddagger^{*}$ & -10882.79579 & -10882.41145 & -10882.34735 & -10882.3464 & -10882.49863 \\
\hline$(\mathrm{C}-\mathrm{P}) \ddagger^{*}$ & -10882.84101 & -10882.45373 & -10882.39004 & -10882.38909 & -10882.54101 \\
\hline$(\mathrm{F}-\mathrm{B}) \ddagger^{*}$ & -10882.88057 & -10882.49449 & -10882.42947 & -10882.42852 & -10882.58392 \\
\hline$(\mathrm{F}-\mathrm{L}) \ddagger^{*}$ & -10882.85798 & -10882.47533 & -10882.41132 & -10882.41037 & -10882.56201 \\
\hline$(\mathrm{G}-\mathrm{B}) \ddagger^{*}$ & -10882.82988 & -10882.44651 & -10882.38197 & -10882.38103 & -10882.53474 \\
\hline$(\mathrm{G}-\mathrm{L}) \ddagger^{*}$ & -10882.87619 & -10882.48605 & -10882.42319 & -10882.42225 & -10882.57114 \\
\hline$(\mathrm{R}-\mathrm{J}) \ddagger^{*}$ & -10882.81366 & -10882.43267 & -10882.36733 & -10882.36638 & -10882.52152 \\
\hline$(\mathrm{J}-\mathrm{K}) \ddagger^{*}$ & -10882.83226 & -10882.44743 & -10882.38202 & -10882.38108 & -10882.53679 \\
\hline$(\mathrm{K}-\mathrm{L}) \ddagger^{*}$ & -10882.82238 & -10882.438 & -10882.37317 & -10882.37222 & -10882.5261 \\
\hline$(\mathrm{L}-\mathrm{M}) \ddagger^{*}$ & -10882.80967 & -10882.42329 & -10882.35954 & -10882.3586 & -10882.51014 \\
\hline$(\mathrm{M}-\mathrm{P}) \ddagger^{*}$ & -10882.85436 & -10882.47067 & -10882.40682 & -10882.40587 & -10882.55798 \\
\hline
\end{tabular}

All reported quantities are in units of Hartrees. Thermal corrections for U, H, and G were calculated at $298 \mathrm{~K}$. 
Table S5. Intrinsic Activation Energies along Pathways 1 and 2 in the Gas Phase and in FAU from Augmented Calculations

\begin{tabular}{|c|c|c|c|c|c|c|}
\hline Pathway & $\begin{array}{c}\text { Intermediate } \\
1\end{array}$ & $\begin{array}{c}\text { Intermediate } \\
2\end{array}$ & $\begin{array}{c}\Delta \mathrm{G}^{\circ \ddagger}(298 \mathrm{~K}) \\
\text { Gas Phase }\end{array}$ & $\begin{array}{c}\Delta \mathrm{G}^{\circ \ddagger}(298 \mathrm{~K}) \\
\text { FAU }\end{array}$ & $\begin{array}{c}\Delta \mathrm{G}^{\ddagger}(498 \mathrm{~K}) \\
\text { Gas Phase }\end{array}$ & $\begin{array}{c}\Delta \mathrm{G}^{\ddagger}(498 \mathrm{~K}) \\
\text { FAU }\end{array}$ \\
\hline $1^{*}$ & $\mathrm{R}$ & $\mathrm{A}$ & & 20.7 & & 23.0 \\
\hline 1 & A & B & 25.9 & 38.5 & 27.2 & 40.7 \\
\hline 1 & B & C & 53.2 & 47.6 & 54.2 & 48.5 \\
\hline Bridge $^{\ddagger}$ & B & $\mathrm{F}$ & & $5 \cdot 4$ & & 8.2 \\
\hline Bridge & B & G & & 25.0 & & $27 \cdot 3$ \\
\hline $1^{\ddagger}$ & $\mathrm{C}$ & $\mathrm{P}$ & & 18.4 & & 22.2 \\
\hline Bridge & $\mathrm{B}$ & $\mathrm{L}$ & 32.7 & & 34.2 & \\
\hline $2^{*}$ & $\mathrm{R}$ & $\mathrm{J}$ & & 20.7 & & 23.0 \\
\hline 2 & $\mathrm{~J}$ & $\mathrm{~K}$ & 12.8 & 11.1 & 13.7 & 13.6 \\
\hline 2 & $\mathrm{~K}$ & $\mathrm{~L}$ & 6.6 & 13.7 & 6.1 & 16.5 \\
\hline 2 & $\mathrm{~L}$ & $\mathrm{M}$ & $25 \cdot 3$ & 32.8 & 25.0 & 31.8 \\
\hline Bridge $^{\ddagger}$ & $\mathrm{L}$ & $\mathrm{F}$ & & 0.6 & & 0.1 \\
\hline Bridge & $\mathrm{L}$ & $\mathrm{G}$ & & 18.6 & & 18.8 \\
\hline $2^{\ddagger}$ & M & $\mathrm{P}$ & & 3.4 & & $5 \cdot 5$ \\
\hline
\end{tabular}

All reported quantities are in units of $\mathrm{kcal} / \mathrm{mol}$. Activation energies were calculated from Single Point calculations at the mo62x/6-311g(3df,2pd) level of theory augmented with the thermal corrections from mo62x/6-31g(d,p) level of theory.

* Denotes protonation reaction $\ddagger$ Denotes deprotonation reaction 
Table S6. DFT Energies for key intermediates in the Gas Phase at 298 K: mo62x/6-311g(3df,2pd)

\begin{tabular}{|l|l|l|l|l|l|}
\hline Intermediate or TS & Electronic Energy & $\begin{array}{l}\text { Zero-Point } \\
\text { Corrected }\end{array}$ & Energy (U) & Enthalpy (H) & Free Energy (G) \\
\hline R & -233.3527161 & -233.2326113 & -233.2259413 & -233.2249973 & -233.2628523 \\
\hline A & -233.6769046 & -233.5436949 & -233.5372509 & -233.5363059 & -233.5735409 \\
\hline B & -233.723731 & -233.5908489 & -233.5846659 & -233.5837219 & -233.6205159 \\
\hline C & -233.6934667 & -233.55868 & -233.553201 & -233.552257 & -233.587153 \\
\hline F & -233.3823389 & -233.2608189 & -233.2550669 & -233.2541229 & -233.2896859 \\
\hline J & -233.6857886 & -233.5539834 & -233.5467254 & -233.5457814 & -233.5849444 \\
\hline K & -233.6796759 & -233.5478863 & -233.5409713 & -233.5400263 & -233.5782503 \\
\hline L & -233.7057601 & -233.5720617 & -233.5661107 & -233.5651667 & -233.6014267 \\
\hline M & -233.706364 & -233.571595 & -233.56605 & -233.565106 & -233.600202 \\
\hline P & -233.3805246 & -233.2570073 & -233.2518423 & -233.2508983 & -233.2852353 \\
\hline
\end{tabular}

All reported quantities are in units of Hartrees. Single Point electronic energies were calculated at the mo62x/6$3119(3 \mathrm{df}, 2 \mathrm{pd})$ level of theory. Thermal corrections for U, H, and G were calculated at $298 \mathrm{~K}$.

Table S7. DFT Energies for key intermediates in the Gas Phase at 498 K: mo62x/6-311g(3df,2pd)

\begin{tabular}{|l|l|l|l|l|l|}
\hline Intermediate or TS & Electronic Energy & $\begin{array}{l}\text { Zero-Point } \\
\text { Corrected }\end{array}$ & Energy (U) & Enthalpy (H) & Free Energy (G) \\
\hline R & -233.3527161 & -233.2326113 & -233.2162433 & -233.2146663 & -233.2908713 \\
\hline A & -233.6769046 & -233.5436949 & -233.5273229 & -233.5257459 & -233.6011789 \\
\hline B & -233.723731 & -233.5908489 & -233.5750639 & -233.5734869 & -233.6477639 \\
\hline C & -233.6934667 & -233.55868 & -233.543836 & -233.542258 & -233.613048 \\
\hline F & -233.3823389 & -233.2608189 & -233.2459179 & -233.2443409 & -233.3159979 \\
\hline J & -233.6857886 & -233.5539824 & -233.5365654 & -233.5349884 & -233.6139604 \\
\hline K & -233.6796759 & -233.5478863 & -233.5308363 & -233.5292583 & -233.6066233 \\
\hline L & -233.7057601 & -233.5720617 & -233.5566117 & -233.5550347 & -233.6282827 \\
\hline M & -233.706364 & -233.571595 & -233.556789 & -233.555212 & -233.62621 \\
\hline P & -233.3805246 & -233.2570073 & -233.2430853 & -233.2415083 & -233.3106093 \\
\hline
\end{tabular}

All reported quantities are in units of Hartrees. Single Point electronic energies were calculated at the mo62x/6$3119(3 \mathrm{df}, 2 \mathrm{pd})$ level of theory. Thermal corrections for U, H, and G were calculated at $498 \mathrm{~K}$. 
Table S8. DFT Energies for key intermediates in FAU at 298K: mo62x/6-311g(3df,2pd)

\begin{tabular}{|l|l|l|l|l|l|}
\hline Intermediate or TS & Electronic Energy & $\begin{array}{l}\text { Zero-Point } \\
\text { Corrected }\end{array}$ & Energy (U) & Enthalpy (H) & Free Energy (G) \\
\hline Zeolite Cluster & -10651.84719 & -10651.58415 & -10651.52589 & -10651.52495 & -10651.66546 \\
\hline$R^{*}$ & -10885.22506 & -10884.84016 & -10884.77435 & -10884.7734 & -10884.92918 \\
\hline$A^{*}$ & -10885.22051 & -10884.83102 & -10884.76612 & -10884.76518 & -10884.92114 \\
\hline$B^{*}$ & -10885.24459 & -10884.85783 & -10884.79309 & -10884.79215 & -10884.94588 \\
\hline$C^{*}$ & -10885.24513 & -10884.85329 & -10884.79028 & -10884.78934 & -10884.93913 \\
\hline$F^{*}$ & -10885.25656 & -10884.8711 & -10884.80595 & -10884.805 & -10884.96024 \\
\hline$G^{*}$ & -10885.24381 & -10884.85274 & -10884.78939 & -10884.78845 & -10884.93786 \\
\hline$J^{*}$ & -10885.22757 & -10884.83976 & -10884.7747 & -10884.77376 & -10884.92825 \\
\hline$K^{*}$ & -10885.22107 & -10884.83329 & -10884.76846 & -10884.76751 & -10884.92034 \\
\hline$L^{*}$ & -10885.23357 & -10884.84667 & -10884.7818 & -10884.78086 & -10884.93554 \\
\hline$M^{*}$ & -10885.23218 & -10884.84415 & -10884.77963 & -10884.77868 & -10884.93257 \\
\hline $\mathrm{P}^{*}$ & -10885.25461 & -10884.86607 & -10884.80155 & -10884.80061 & -10884.95525 \\
\hline
\end{tabular}

All reported quantities are in units of Hartrees. Single Point electronic energies were calculated at the mo62x/6$311 \mathrm{~g}(3 \mathrm{df}, 2 \mathrm{pd})$ level of theory. Thermal corrections for U, H, and G were calculated at $298 \mathrm{~K}$.

Table S9. DFT Energies for key intermediates in FAU at 498K: mo62x/6-311g(3df,2pd)

\begin{tabular}{|l|l|l|l|l|l|}
\hline Intermediate or TS & Electronic Energy & $\begin{array}{l}\text { Zero-Point } \\
\text { Corrected }\end{array}$ & Energy (U) & Enthalpy (H) & Free Energy (G) \\
\hline Zeolite Cluster & -10651.84719 & -10651.58415 & -10651.4375 & -10651.43592 & -10651.78311 \\
\hline $\mathrm{R}^{*}$ & -10885.22506 & -10884.84015 & -10884.67467 & -10884.67309 & -10885.05992 \\
\hline $\mathrm{A}^{*}$ & -10885.22051 & -10884.83102 & -10884.66747 & -10884.66589 & -10885.05175 \\
\hline $\mathrm{B}^{*}$ & -10885.24459 & -10884.85783 & -10884.69424 & -10884.69266 & -10885.07506 \\
\hline $\mathrm{C}^{*}$ & -10885.24513 & -10884.8533 & -10884.69237 & -10884.6908 & -10885.06538 \\
\hline $\mathrm{F}^{*}$ & -10885.25656 & -10884.8711 & -10884.70682 & -10884.70525 & -10885.09047 \\
\hline $\mathrm{G}^{*}$ & -10885.24381 & -10884.85274 & -10884.69113 & -10884.68956 & -10885.06394 \\
\hline $\mathrm{J}^{*}$ & -10885.22757 & -10884.83976 & -10884.6757 & -10884.67413 & -10885.05796 \\
\hline $\mathrm{K}^{*}$ & -10885.22107 & -10884.83329 & -10884.66942 & -10884.66784 & -10885.04896 \\
\hline $\mathrm{L}^{*}$ & -10885.23357 & -10884.84668 & -10884.68301 & -10884.68143 & -10885.06538 \\
\hline $\mathrm{M}^{*}$ & -10885.23218 & -10884.84415 & -10884.68104 & -10884.67946 & -10885.06175 \\
\hline $\mathrm{P}^{*}$ & -10885.25461 & -10884.86608 & -10884.70286 & -10884.70128 & -10885.08491 \\
\hline
\end{tabular}

All reported quantities are in units of Hartrees. Single Point electronic energies were calculated at the mo62x/6$3119(3 \mathrm{df}, 2 \mathrm{pd})$ level of theory. Thermal corrections for U, H, and G were calculated at $498 \mathrm{~K}$.

Table S1o. DFT Energies for transition states in the Gas Phase at 298K: mo62x/6-311g(3df,2pd)

\begin{tabular}{|l|l|l|l|l|l|}
\hline Intermediate or TS & Electronic Energy & $\begin{array}{l}\text { Zero-Point } \\
\text { Corrected }\end{array}$ & Energy (U) & Enthalpy (H) & Free Energy (G) \\
\hline$(\mathrm{A}-\mathrm{B}) \ddagger$ & -233.6335407 & -233.5033421 & -233.4975891 & -233.4966441 & -233.5322292 \\
\hline$(\mathrm{B}-\mathrm{C}) \ddagger$ & -233.6374111 & -233.5064039 & -233.5007359 & -233.4997919 & -233.5357409 \\
\hline$(\mathrm{B}-\mathrm{L}) \ddagger$ & -233.669209 & -233.5396881 & -233.5340601 & -233.5331161 & -233.5684431 \\
\hline$(\mathrm{J}-\mathrm{K}) \ddagger$ & -233.6639768 & -233.533958 & -233.527162 & -233.526218 & -233.564482 \\
\hline$(\mathrm{K}-\mathrm{L}) \ddagger$ & -233.6699914 & -233.5385689 & -233.5326339 & -233.5316899 & -233.5677669 \\
\hline$(\mathrm{L}-\mathrm{M}) \ddagger$ & -233.6660019 & -233.5331879 & -233.5282819 & -233.5273379 & -233.5611499 \\
\hline
\end{tabular}


All reported quantities are in units of Hartrees. Single Point electronic energies were calculated at the mo62x/6$311 \mathrm{~g}(3 \mathrm{df}, 2 \mathrm{pd})$ level of theory. Thermal corrections for U, H, and G were calculated at $298 \mathrm{~K}$. 
Table S11. DFT Energies for transition states in the Gas Phase at 498K: mo62x/6-311g(3df,2pd)

\begin{tabular}{|l|l|l|l|l|l|}
\hline Intermediate or TS & Electronic Energy & $\begin{array}{l}\text { Zero-Point } \\
\text { Corrected }\end{array}$ & Energy (U) & Enthalpy (H) & Free Energy (G) \\
\hline$(\mathrm{A}-\mathrm{B}) \ddagger$ & -233.6335407 & -233.5033421 & -233.4881201 & -233.4865431 & -233.5586311 \\
\hline$(\mathrm{B}-\mathrm{C}) \ddagger$ & -233.6374111 & -233.5064039 & -233.4914549 & -233.4898779 & -233.5623309 \\
\hline$(\mathrm{B}-\mathrm{L}) \ddagger$ & -233.669209 & -233.5396881 & -233.5246911 & -233.5231141 & -233.5946391 \\
\hline$(\mathrm{J}-\mathrm{K}) \ddagger$ & -233.6639768 & -233.533958 & -233.517416 & -233.515839 & -233.592783 \\
\hline$(\mathrm{K}-\mathrm{L}) \ddagger$ & -233.6699914 & -233.5385689 & -233.5231779 & -233.5216009 & -233.5945019 \\
\hline$(\mathrm{L}-\mathrm{M}) \ddagger$ & -233.6660019 & -233.5331879 & -233.5194939 & -233.5179169 & -233.5861579 \\
\hline
\end{tabular}

All reported quantities are in units of Hartrees. Single Point electronic energies were calculated at the mo62x/6$3119(3 \mathrm{df}, 2 \mathrm{pd})$ level of theory. Thermal corrections for U, H, and G were calculated at $498 \mathrm{~K}$.

Table S12. DFT Energies for transition states in FAU at 298K: mo62x/6-311g(3df,2pd)

\begin{tabular}{|l|l|l|l|l|l|}
\hline Intermediate or TS & Electronic Energy & $\begin{array}{l}\text { Zero-Point } \\
\text { Corrected }\end{array}$ & Energy (U) & Enthalpy (H) & Free Energy (G) \\
\hline$(\mathrm{R}-\mathrm{A}) \ddagger^{*}$ & -10885.18838 & -10884.80739 & -10884.74204 & -10884.7411 & -10884.89623 \\
\hline$(\mathrm{A}-\mathrm{B}) \ddagger^{*}$ & -10885.15559 & -10884.77159 & -10884.70705 & -10884.70611 & -10884.85984 \\
\hline$(\mathrm{B}-\mathrm{C}) \ddagger^{*}$ & -10885.16724 & -10884.7829 & -10884.71879 & -10884.71785 & -10884.87008 \\
\hline$(\mathrm{C}-\mathrm{P}) \ddagger^{*}$ & -10885.20975 & -10884.82247 & -10884.75878 & -10884.75783 & -10884.90975 \\
\hline$(\mathrm{F}-\mathrm{B}) \ddagger^{*}$ & -10885.23159 & -10884.84999 & -10884.78579 & -10884.78484 & -10884.93721 \\
\hline$(\mathrm{F}-\mathrm{L}) \ddagger^{*}$ & -10885.2266 & -10884.84395 & -10884.77993 & -10884.77899 & -10884.93063 \\
\hline$(\mathrm{G}-\mathrm{B}) \ddagger^{*}$ & -10885.20113 & -10884.81777 & -10884.75322 & -10884.75228 & -10884.90599 \\
\hline$(\mathrm{G}-\mathrm{L}) \ddagger^{*}$ & -10885.24384 & -10884.85369 & -10884.79084 & -10884.78989 & -10884.93878 \\
\hline$(\mathrm{R}-\mathrm{J}) \ddagger^{*}$ & -10885.18838 & -10884.80739 & -10884.74205 & -10884.7411 & -10884.89624 \\
\hline$(\mathrm{J}-\mathrm{K}) \ddagger^{*}$ & -10885.20596 & -10884.82112 & -10884.75571 & -10884.75477 & -10884.91049 \\
\hline$(\mathrm{K}-\mathrm{L}) \ddagger^{*}$ & -10885.19484 & -10884.81046 & -10884.74563 & -10884.74469 & -10884.89856 \\
\hline$(\mathrm{L}-\mathrm{M}) \ddagger^{*}$ & -10885.18285 & -10884.79647 & -10884.73272 & -10884.73178 & -10884.88331 \\
\hline$(\mathrm{M}-\mathrm{P}) \ddagger^{*}$ & -10885.22348 & -10884.83979 & -10884.77594 & -10884.775 & -10884.92711 \\
\hline
\end{tabular}

All reported quantities are in units of Hartrees. Single Point electronic energies were calculated at the mo62x/6$3119(3 \mathrm{df}, 2 \mathrm{pd})$ level of theory. Thermal corrections for U, H, and G were calculated at $298 \mathrm{~K}$. 
Table S13. DFT Energies for transition states in FAU at 498K: mo62x/6-311g(3df,2pd)

\begin{tabular}{|l|l|l|l|l|l|}
\hline Intermediate or TS & Electronic Energy & $\begin{array}{l}\text { Zero-Point } \\
\text { Corrected }\end{array}$ & Energy (U) & Enthalpy (H) & Free Energy (G) \\
\hline$(\mathrm{R}-\mathrm{A}) \ddagger^{*}$ & -10885.18838 & -10884.80739 & -10884.64294 & -10884.64136 & -10885.02642 \\
\hline$(\mathrm{A}-\mathrm{B}) \ddagger^{*}$ & -10885.15559 & -10884.77159 & -10884.60836 & -10884.60678 & -10884.98896 \\
\hline$(\mathrm{B}-\mathrm{C}) \ddagger^{*}$ & -10885.16724 & -10884.7829 & -10884.62034 & -10884.61877 & -10884.99811 \\
\hline$(\mathrm{C}-\mathrm{P}) \ddagger^{*}$ & -10885.20975 & -10884.82247 & -10884.6607 & -10884.65912 & -10885.03746 \\
\hline$(\mathrm{F}-\mathrm{B}) \ddagger^{*}$ & -10885.23159 & -10884.84999 & -10884.6872 & -10884.68563 & -10885.06537 \\
\hline$(\mathrm{F}-\mathrm{L}) \ddagger^{*}$ & -10885.2266 & -10884.84395 & -10884.68149 & -10884.67992 & -10885.05826 \\
\hline$(\mathrm{G}-\mathrm{B}) \ddagger^{*}$ & -10885.20113 & -10884.81777 & -10884.6546 & -10884.65302 & -10885.03508 \\
\hline$(\mathrm{G}-\mathrm{L}) \ddagger^{*}$ & -10885.24384 & -10884.85369 & -10884.69313 & -10884.69155 & -10885.06436 \\
\hline$(\mathrm{R}-\mathrm{J}) \ddagger^{*}$ & -10885.18838 & -10884.80739 & -10884.64294 & -10884.64136 & -10885.02644 \\
\hline$(\mathrm{J}-\mathrm{K}) \ddagger^{*}$ & -10885.20596 & -10884.82112 & -10884.65684 & -10884.65527 & -10885.041 \\
\hline$(\mathrm{K}-\mathrm{L}) \ddagger^{*}$ & -10885.19484 & -10884.81046 & -10884.6468 & -10884.64522 & -10885.02782 \\
\hline$(\mathrm{L}-\mathrm{M}) \ddagger^{*}$ & -10885.18285 & -10884.79647 & -10884.63461 & -10884.63304 & -10885.01078 \\
\hline$(\mathrm{M}-\mathrm{P}) \ddagger^{*}$ & -10885.22348 & -10884.83979 & -10884.67774 & -10884.67616 & -10885.05498 \\
\hline
\end{tabular}

All reported quantities are in units of Hartrees. Single Point electronic energies were calculated at the mo62x/6$311 \mathrm{~g}(3 \mathrm{df}, 2 \mathrm{pd})$ level of theory. Thermal corrections for $\mathrm{U}, \mathrm{H}$, and $\mathrm{G}$ were calculated at $498 \mathrm{~K}$.

Table S14. Microkinetic Model Parameters

\begin{tabular}{|l|l|}
\hline Parameter & Value \\
\hline Temperature & $298 \mathrm{~K}$ \\
\hline Pressure & 1 atm \\
\hline Volumetric Flowrate & $0.5 \mathrm{~cm}^{3} / \mathrm{s}$ \\
\hline Catalyst Site Density & $1.893 \mathrm{e}-10 \mathrm{~mol} / \mathrm{cm}$ \\
\hline Catalyst Density & $1.325 \mathrm{~g} / \mathrm{cm}^{3}$ \\
\hline Catalyst Area/Volume & $9.005 \mathrm{~cm}^{-1}$ \\
\hline Reaction Conditions & Isothermal \\
\hline Feed Composition & Pure Hexatriene Gas \\
\hline Reactor Type & Single CSTR \\
\hline
\end{tabular}

Input files (.inp) for specific MKM runs have been included in the Supporting Information as a .zip file.

\section{S1 Optimized Geometries at the Mo62X/6-31g(d,p) level of theory}

R (Gas Phase)

(Electronic Energy: -233.279344815 Ha)
C
C
$\begin{array}{lll}-3.74555800 & -0.04775800 & -0.43515800\end{array}$
$-2.41179800 \quad-0.12026400-0.40162600$
$\begin{array}{llll}-4.26616300 & 0.90308100 & -0.43173100\end{array}$
$\begin{array}{llll}-4.35593900 & -0.94628000 & -0.46595400\end{array}$
$\begin{array}{lll}-1.68731100 & -1.38515700 & -0.40617200\end{array}$
$\begin{array}{lll}-1.83475200 & 0.80052500 & -0.37098800\end{array}$
$\begin{array}{llll}-2.30916600 & -2.27798800 & -0.44133400\end{array}$
$\begin{array}{llll}-0.35119600 & -1.54387300 & -0.37093300\end{array}$
$\begin{array}{lll}0.04531500 & -2.55774200 & -0.38120800\end{array}$
$\begin{array}{llll}0.64871300 & -0.48436900 & -0.31812000\end{array}$ 


$$
\begin{array}{llll}
\mathrm{C} & 1.96205300 & -0.72810200 & -0.28644300 \\
\mathrm{H} & 0.30317100 & 0.54632100 & -0.30282900 \\
\mathrm{H} & 2.69089000 & 0.07337700 & -0.24535300 \\
\mathrm{H} & 2.34459400 & -1.74510600 & -0.30030800
\end{array}
$$

A (Gas Phase)

(Electronic Energy: -233.610201692 Ha):

$\begin{array}{llcc}\mathrm{C} & -1.58740600 & 0.02034300 & -1.07744300 \\ \mathrm{C} & -1.80625300 & 0.46894900 & 0.27214300 \\ \mathrm{H} & -1.23033700 & -0.98527300 & -1.26839600 \\ \mathrm{H} & -2.49942300 & 1.28109600 & 0.46270900 \\ \mathrm{C} & -0.50544100 & 1.00282300 & -0.46136100 \\ \mathrm{H} & -1.61565900 & -0.19613900 & 1.10675300 \\ \mathrm{H} & -0.54316700 & 2.02678100 & -0.80814200 \\ \mathrm{C} & 0.73084100 & 0.47676600 & -0.08558100 \\ \mathrm{C} & 0.98979400 & -0.83748300 & 0.39430000 \\ \mathrm{H} & 1.59469900 & 1.13826500 & -0.16531700 \\ \mathrm{C} & 2.25853600 & -1.17967400 & 0.71170800 \\ \mathrm{H} & -2.11633000 & 0.49820200 & -1.89487700 \\ \mathrm{H} & 0.18280800 & -1.55605900 & 0.50406500 \\ \mathrm{H} & 3.07730200 & -0.47187200 & 0.60774100 \\ \mathrm{H} & 2.50426600 & -2.17069300 & 1.08053600\end{array}$

B (Gas Phase)

(Electronic Energy: -233.659934157 Ha):

$\begin{array}{llll}\mathrm{C} & -0.69945700 & -1.50132200 & -0.29938100 \\ \mathrm{C} & 0.70707700 & -1.25886100 & -0.68828700 \\ \mathrm{C} & 1.11733900 & 0.02373700 & -0.41135700 \\ \mathrm{C} & 0.04505800 & 0.71827600 & 0.16982300 \\ \mathrm{C} & -1.15142900 & -0.15517300 & 0.26826500 \\ \mathrm{H} & -1.28502100 & -1.83535600 & -1.16571800 \\ \mathrm{H} & -0.75494800 & -2.32410000 & 0.42594500 \\ \mathrm{H} & 1.33553900 & -2.02161600 & -1.14178400 \\ \mathrm{H} & 2.09896800 & 0.43921100 & -0.60315400 \\ \mathrm{H} & -1.49886100 & -0.20399600 & 1.30885200 \\ \mathrm{H} & -1.97932900 & 0.29815200 & -0.29387600 \\ \mathrm{C} & 0.08472700 & 2.10646100 & 0.65714300 \\ \mathrm{H} & 0.85923000 & 2.69554800 & 0.16438200 \\ \mathrm{H} & -0.88925600 & 2.59434100 & 0.58163400 \\ \mathrm{H} & 0.33608600 & 2.06345300 & 1.72902900\end{array}$

C (Gas Phase)

(Electronic Energy: -233.629233719 Ha):

\begin{tabular}{|c|c|}
\hline $\mathrm{C}$ & $-0.76390600 \quad-1.16436700 \quad-0.50947000$ \\
\hline $\mathrm{C}$ & $\begin{array}{lll}0.69451300 & -0.92957100 & -0.68375400\end{array}$ \\
\hline $\mathrm{C}$ & $\begin{array}{lll}1.17996600 & 0.21343100 & -0.10828100\end{array}$ \\
\hline $\mathrm{C}$ & $\begin{array}{lll}-0.04981100 & 0.69250300 & 0.89741800\end{array}$ \\
\hline $\mathrm{C}$ & $-1.15375800 \quad-0.33780400 \quad 0.73243000$ \\
\hline $\mathrm{H}$ & $\begin{array}{lll}-1.28584300 & -0.88628900 & -1.43893900\end{array}$ \\
\hline $\mathrm{H}$ & $-0.96633800 \quad-2.23112200 \quad-0.37708500$ \\
\hline $\mathrm{H}$ & $1.34699400 \quad-1.65636700 \quad-1.16557900$ \\
\hline $\mathrm{H}$ & $\begin{array}{ccc}2.22968000 & 0.41726900 & 0.05572400\end{array}$ \\
\hline
\end{tabular}




$$
\begin{array}{cccc}
\mathrm{H} & -1.19849100 & -0.98456900 & 1.60966200 \\
\mathrm{H} & -2.12138500 & 0.15963600 & 0.63044200 \\
\mathrm{C} & 0.24063600 & 1.51542100 & -0.22892800 \\
\mathrm{H} & 0.85833800 & 2.39939200 & -0.11004500 \\
\mathrm{H} & -0.40920400 & 1.49902100 & -1.09872100 \\
\mathrm{H} & 0.31761600 & 0.97072300 & 1.88085600
\end{array}
$$

F (Gas Phase)

$$
\begin{array}{llll}
\text { (Electronic Energy: }-233.313008029 \mathrm{Ha}): \\
\mathrm{C} & -3.79465100 & -0.71560200 & -0.56592200 \\
\mathrm{C} & -2.46769300 & -0.62189500 & -0.75310800 \\
\mathrm{C} & -1.98833300 & 0.65315900 & -0.20221700 \\
\mathrm{C} & -3.02209200 & 1.33814500 & 0.32171400 \\
\mathrm{C} & -4.27663900 & 0.52245000 & 0.13613200 \\
\mathrm{H} & -4.43699700 & -1.53555500 & -0.86050600 \\
\mathrm{H} & -1.83479600 & -1.35996500 & -1.23192900 \\
\mathrm{H} & -0.95591600 & 0.98517000 & -0.21905100 \\
\mathrm{H} & -4.75176100 & 0.29166200 & 1.09954500 \\
\mathrm{H} & -5.02665800 & 1.06662000 & -0.45410300 \\
\mathrm{C} & -3.00540200 & 2.67670800 & 0.98660800 \\
\mathrm{H} & -1.99334900 & 3.08679100 & 1.01540100 \\
\mathrm{H} & -3.64697100 & 3.39149000 & 0.45927800 \\
\mathrm{H} & -3.37611600 & 2.61457000 & 2.01580000
\end{array}
$$

\section{J (Gas Phase)}

(Electronic Energy: -233.619303193 Ha):

$\begin{array}{llll}\mathrm{C} & -3.73886100 & 1.22409800 & -0.23824500 \\ \mathrm{C} & -2.86370000 & 0.61840000 & 0.59829900 \\ \mathrm{H} & -3.72482500 & 1.03044800 & -1.30797600 \\ \mathrm{H} & -4.48371400 & 1.92285900 & 0.12921900 \\ \mathrm{C} & -1.91189200 & -0.28449500 & 0.05262100 \\ \mathrm{H} & -2.89673600 & 0.82671500 & 1.66218200 \\ \mathrm{H} & -1.96191400 & -0.42191100 & -1.02792600 \\ \mathrm{C} & -0.92283300 & -1.01545900 & 0.72539100 \\ \mathrm{C} & -0.73900500 & -0.95141600 & 2.08530900 \\ \mathrm{H} & -0.27772700 & -1.66042700 & 0.13674300 \\ \mathrm{C} & 0.28490400 & -1.70790200 & 2.82052000 \\ \mathrm{H} & -1.38412900 & -0.30574600 & 2.68026800 \\ \mathrm{H} & 0.91112500 & -1.00919200 & 3.39148000 \\ \mathrm{H} & -0.20695100 & -2.33265100 & 3.57894700 \\ \mathrm{H} & 0.90691200 & -2.32670900 & 2.17517000\end{array}$

\section{K (Gas Phase)}

(Electronic Energy: -233.613613516 Ha):

$\begin{array}{llll}\mathrm{C} & -1.87176000 & -0.23797600 & 0.93080400 \\ \mathrm{C} & -1.33676300 & 1.00444900 & 0.86444300 \\ \mathrm{H} & -1.24579600 & -1.12491100 & 0.96794300 \\ \mathrm{H} & -2.94249800 & -0.37899800 & 1.04197200 \\ \mathrm{C} & 0.06917100 & 1.21705900 & 0.76260500 \\ \mathrm{H} & -1.97034900 & 1.87453100 & 1.01716500 \\ \mathrm{H} & 0.47179200 & 2.10788000 & 1.24565500\end{array}$




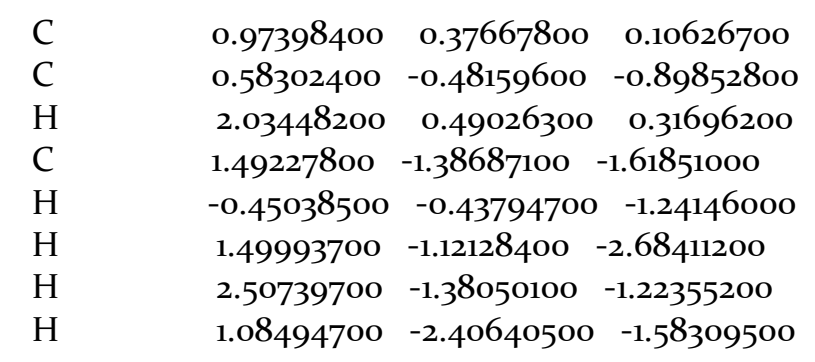

L (Gas Phase)

(Electronic Energy: -233.641704445 Ha):

$\begin{array}{lccl}C & 0.25250500 & -0.21059100 & 0.89445400 \\ C & 0.02197100 & 0.50044900 & 2.16351600 \\ C & -1.27427400 & 0.98238700 & 2.26764400 \\ \text { C } & -1.93676800 & 0.63585100 & 1.09837700 \\ H & 1.09935600 & 0.23660700 & 0.35419900 \\ H & 0.56245300 & -1.24678600 & 1.09912800 \\ H & 0.78930600 & 0.62934600 & 2.92353900 \\ H & -1.68931800 & 1.53031400 & 3.10371300 \\ H & -2.97605200 & 0.87886200 & 0.88433600 \\ C & -1.08855300 & -0.11958100 & 0.15654100 \\ C & -1.71754800 & -1.47532800 & -0.24325400 \\ H & -0.99738200 & 0.48515100 & -0.76046200 \\ H & -1.05793700 & -1.97878300 & -0.95121000 \\ H & -1.84717500 & -2.11362600 & 0.63352100 \\ H & -2.68697900 & -1.32678700 & -0.72102200\end{array}$

M (Gas Phase)

(Electronic Energy: -233.642557009 Ha):

$\begin{array}{lccc}\mathrm{C} & 0.24646100 & -1.40171200 & -0.13840000 \\ \mathrm{C} & 1.29703400 & -0.62834300 & 0.33015900 \\ \mathrm{C} & 1.04127300 & 0.70466900 & 0.61162000 \\ \mathrm{C} & -1.06741200 & -0.85042700 & -0.51566200 \\ \mathrm{H} & 0.37462000 & -2.48364400 & -0.17296800 \\ \mathrm{H} & 2.24262000 & -1.08236600 & 0.60287400 \\ \mathrm{H} & 1.79129300 & 1.27042200 & 1.16452000 \\ \mathrm{H} & -1.73444100 & -1.09667400 & 0.33397600 \\ \mathrm{H} & -1.47769300 & -1.42649700 & -1.35265800 \\ \mathrm{C} & -1.02620300 & 0.64992000 & -0.79762000 \\ \mathrm{H} & -0.55013600 & 0.81526600 & -1.76908300 \\ \mathrm{C} & -0.22521600 & 1.38096400 & 0.27757100 \\ \mathrm{H} & -0.02184900 & 2.42553400 & 0.01648000 \\ \mathrm{H} & -2.03727500 & 1.05375200 & -0.86000700 \\ \mathrm{H} & -0.77629900 & 1.44527300 & 1.23659400\end{array}$

P (Gas Phase)

(Electronic Energy: -233.311009304 Ha):

$\begin{array}{llll}C & -0.45609200 & 0.56576000 & -0.01595800 \\ C & -1.15327500 & 1.70766000 & -0.00830300 \\ C & -2.59508700 & 1.70150400 & -0.29350600 \\ C & -3.28790700 & 0.55746200 & -0.25846800\end{array}$




$$
\begin{array}{llll}
H & 0.61573600 & 0.56814600 & 0.16092900 \\
H & -0.66352700 & 2.65693100 & 0.18785600 \\
H & -3.08860400 & 2.64392800 & -0.51194700 \\
H & -4.35979100 & 0.55168700 & -0.43496600 \\
C & -2.59984200 & -0.73454700 & 0.10744300 \\
H & -2.64135500 & -0.85204700 & 1.20120300 \\
C & -1.13913800 & -0.73707400 & -0.35164600 \\
H & -1.09726000 & -0.87983500 & -1.44237200 \\
H & -3.12932800 & -1.59204900 & -0.31757300 \\
H & -0.60644100 & -1.58238400 & 0.09327000
\end{array}
$$

A-B TS (Gas Phase)

(Electronic Energy: -233.566010573 Ha):

$\begin{array}{llll}\mathrm{C} & -0.94321600 & -0.77528400 & -0.53485800 \\ \mathrm{C} & -1.60550700 & 0.55662700 & -0.22548700 \\ \mathrm{H} & -1.48641400 & -1.67324600 & -0.22565200 \\ \mathrm{H} & -2.14287100 & 0.89658800 & -1.11748000 \\ \mathrm{C} & -0.49936000 & 1.50796700 & 0.14816000 \\ \mathrm{H} & -2.34072300 & 0.47064900 & 0.58137300 \\ \mathrm{H} & -0.68931400 & 2.56955300 & 0.25519900 \\ \mathrm{C} & 0.68803200 & 0.93688800 & 0.36259600 \\ \mathrm{C} & 0.68723900 & -0.51719900 & 0.18567600 \\ \mathrm{H} & 1.59505300 & 1.45502300 & 0.64946800 \\ \mathrm{C} & 1.67009200 & -1.43291600 & 0.07775300 \\ \mathrm{H} & -0.58035600 & -0.89456100 & -1.55385000 \\ \mathrm{H} & -0.18649400 & -1.08039100 & 0.69455900 \\ \mathrm{H} & 2.71234600 & -1.13052900 & 0.13921000 \\ \mathrm{H} & 1.45724800 & -2.49121300 & -0.03810800\end{array}$

B-C TS (Gas Phase)

(Electronic Energy: -233.571612205 Ha):

$\begin{array}{lccc}C & -1.41758800 & -2.32240400 & -0.30537200 \\ \mathrm{C} & 0.05283400 & -2.13066400 & -0.56134900 \\ \mathrm{C} & 0.57373600 & -1.02952500 & -0.04183700 \\ \mathrm{C} & -0.57394700 & -0.26525000 & 0.72016300 \\ \mathrm{C} & -1.78879400 & -1.20219100 & 0.69478700 \\ \mathrm{H} & -1.97508500 & -2.24431100 & -1.24439300 \\ \mathrm{H} & -1.62504900 & -3.31412800 & 0.10315700 \\ \mathrm{H} & 0.64625100 & -2.83884900 & -1.13245900 \\ \mathrm{H} & 1.58642200 & -0.65054300 & -0.07093700 \\ \mathrm{H} & -1.94466300 & -1.61144900 & 1.69424000 \\ \mathrm{H} & -2.70112500 & -0.67100000 & 0.41413900 \\ \mathrm{C} & -0.68497000 & 1.08001600 & 0.32454600 \\ \mathrm{H} & 0.19898100 & 1.71891800 & 0.30259500 \\ \mathrm{H} & -1.63670900 & 1.54459800 & 0.05608300 \\ \mathrm{H} & -0.10066100 & -0.11089600 & 1.73178200\end{array}$

B-L TS (Gas Phase)

(Electronic Energy: -233.603560885 Ha):

$\begin{array}{llll}C & -3.60006700 & -0.21537800 & 0.33718700 \\ C & -2.20970300 & -0.17005600 & 0.02711800\end{array}$ 


$\begin{array}{llll}\mathrm{C} & -1.90538400 & 1.22083500 & -0.41477300 \\ \mathrm{C} & -3.02023000 & 1.95763200 & -0.31271800 \\ \mathrm{C} & -4.17461200 & 1.10976200 & 0.14256700 \\ \mathrm{H} & -4.13920300 & -1.09437400 & 0.68470300 \\ \mathrm{H} & -1.63539600 & -1.05139200 & -0.25343100 \\ \mathrm{H} & -2.17332400 & -0.14536100 & 1.19267700 \\ \mathrm{H} & -0.92229100 & 1.54263000 & -0.72981300 \\ \mathrm{H} & -3.12725000 & 3.01164000 & -0.53724400 \\ \mathrm{C} & -5.22430400 & 1.66827400 & 1.10516800 \\ \mathrm{H} & -5.67989100 & 2.55935800 & 0.67284200 \\ \mathrm{H} & -6.00814800 & 0.93506100 & 1.30011900 \\ \mathrm{H} & -4.74908600 & 1.94403200 & 2.04795900 \\ \mathrm{H} & -4.70041500 & 0.80111200 & -0.80982900\end{array}$

\section{J-K TS (Gas Phase)}

(Electronic Energy: -233.597052767 Ha):

$\begin{array}{llll}\mathrm{C} & -4.78402300 & -2.56616000 & 0.63056300 \\ \mathrm{C} & -3.97381300 & -1.73281500 & -0.01834400 \\ \mathrm{H} & -4.41940900 & -3.26894100 & 1.37335300 \\ \mathrm{H} & -5.84859200 & -2.56206800 & 0.42646400 \\ \mathrm{C} & -2.52434500 & -1.65175700 & 0.21602000 \\ \mathrm{H} & -4.36290800 & -1.01931900 & -0.74695000 \\ \mathrm{H} & -2.19228100 & -1.02911900 & 1.05014700 \\ \mathrm{C} & -1.54461300 & -2.26333400 & -0.54291100 \\ \mathrm{C} & -1.89980200 & -3.11334100 & -1.58265700 \\ \mathrm{H} & -0.49691000 & -2.09842500 & -0.31075800 \\ \mathrm{C} & -0.95388700 & -3.82682100 & -2.43703800 \\ \mathrm{H} & -2.96462900 & -3.26821500 & -1.76518500 \\ \mathrm{H} & -1.17189200 & -3.57898900 & -3.48671200 \\ \mathrm{H} & 0.09025300 & -3.62413300 & -2.20462100 \\ \mathrm{H} & -1.16174200 & -4.90546200 & -2.36907800\end{array}$

K-L TS (Gas Phase)

(Electronic Energy: -233.604140511 Ha):

$\begin{array}{lccc}\mathrm{C} & 0.61823200 & -0.24557100 & 1.28529400 \\ \mathrm{C} & 0.19620800 & 0.86580500 & 1.99288300 \\ \mathrm{C} & -1.18252400 & 1.00005600 & 2.23141100 \\ \mathrm{C} & -1.97317900 & 0.25748600 & 1.36714700 \\ \mathrm{H} & 1.58414300 & -0.26434100 & 0.78545700 \\ \mathrm{H} & 0.15313500 & -1.21476800 & 1.44939100 \\ \mathrm{H} & 0.90377100 & 1.63713400 & 2.28961100 \\ \mathrm{H} & -1.59144200 & 1.62877900 & 3.01623600 \\ \mathrm{H} & -3.00667800 & 0.00841900 & 1.60215500 \\ \mathrm{C} & -1.39733800 & -0.23458900 & 0.18241000 \\ \mathrm{C} & -1.92476600 & -1.40516400 & -0.56334300 \\ \mathrm{H} & -0.73872700 & 0.43420300 & -0.37231400 \\ \mathrm{H} & -1.11714900 & -1.93374000 & -1.07641400 \\ \mathrm{H} & -2.48215200 & -2.09682300 & 0.06808500 \\ \mathrm{H} & -2.58792800 & -1.02940000 & -1.35498800\end{array}$

L-M TS (Gas Phase) 
(Electronic Energy: -233.599937002 Ha):

$\begin{array}{llll}\mathrm{C} & -2.22486200 & -0.36576900 & 0.59447700 \\ \mathrm{C} & -1.26280600 & 0.49806200 & 0.91191700 \\ \mathrm{C} & -1.58511300 & 1.88128300 & 0.42758100 \\ \mathrm{C} & -3.32456000 & 0.25741600 & -0.22889600 \\ \mathrm{H} & -2.24285300 & -1.41331900 & 0.87030000 \\ \mathrm{H} & -0.35328800 & 0.28306600 & 1.45799100 \\ \mathrm{H} & -0.83026900 & 2.49850900 & -0.04918000 \\ \mathrm{H} & -4.32697700 & 0.09539400 & 0.17690000 \\ \mathrm{H} & -3.33198500 & -0.12676200 & -1.25510900 \\ \mathrm{C} & -2.92497800 & 1.73616800 & -0.29912000 \\ \mathrm{H} & -3.06253200 & 2.34849400 & -1.18758300 \\ \mathrm{C} & -2.63153500 & 2.60709500 & 1.17717500 \\ \mathrm{H} & -2.70038400 & 3.68833700 & 1.08089400 \\ \mathrm{H} & -3.72421900 & 2.32201500 & 0.42810000 \\ \mathrm{H} & -3.02307800 & 2.14391500 & 2.08119000\end{array}$

Zeolite Cluster (H-FAU)

(Electronic Energy: -10649.5372823 Ha):

$\begin{array}{lllll}\mathrm{O} & \mathrm{o} & 36.52631400 & 21.73772900 & 14.74988200 \\ \mathrm{O} & \mathrm{o} & 32.99901500 & 12.13124500 & 15.61823400 \\ \mathrm{O} & \mathrm{o} & 33.03569300 & 15.63222900 & 12.18715400 \\ \mathrm{O} & \mathrm{o} & 30.23907800 & 21.03810000 & 14.67795700 \\ \mathrm{O} & \mathrm{o} & 33.00875300 & 14.80904300 & 18.36674400 \\ \mathrm{O} & \mathrm{o} & 27.95180500 & 20.89046300 & 12.26964100 \\ \mathrm{O} & \mathrm{o} & 30.38390500 & 15.73344700 & 9.59739900 \\ \mathrm{O} & \mathrm{o} & 31.14548500 & 16.62793300 & 13.73912900 \\ \mathrm{O} & \mathrm{o} & 28.62520800 & 19.01701000 & 14.02982400 \\ \mathrm{O} & \mathrm{o} & 28.59337800 & 16.40575400 & 11.45141400 \\ \mathrm{O} & \mathrm{o} & 34.82038400 & 22.60811000 & 12.88675800 \\ \mathrm{O} & \mathrm{o} & 26.14434500 & 18.97254700 & 16.46265500 \\ \mathrm{O} & \mathrm{o} & 31.24103900 & 13.84088900 & 16.62492500 \\ \mathrm{O} & \mathrm{o} & 28.60390900 & 14.03125400 & 18.99234800 \\ \mathrm{O} & \mathrm{o} & 29.61221900 & 20.05801600 & 10.36270600 \\ \mathrm{O} & \mathrm{o} & 32.26720300 & 17.45652600 & 10.36179000 \\ \mathrm{O} & \mathrm{o} & 32.08598800 & 19.89843100 & 13.14555600 \\ \mathrm{O} & \mathrm{o} & 30.54516100 & 14.66502400 & 12.05888900 \\ \mathrm{O} & \mathrm{o} & 30.57449600 & 18.62575600 & 15.79426400 \\ \mathrm{O} & \mathrm{o} & 26.95124500 & 18.40481700 & 12.05702900 \\ \mathrm{O} & \mathrm{o} & 36.59275800 & 20.76386100 & 12.24295600 \\ \mathrm{O} & \mathrm{o} & 27.89333600 & 18.34047200 & 18.32920800 \\ \mathrm{O} & \mathrm{o} & 30.50501800 & 15.73191500 & 18.32405100 \\ \mathrm{O} & \mathrm{o} & 30.49155900 & 12.14491900 & 14.71702500 \\ \mathrm{O} & \mathrm{o} & 30.50133600 & 21.78930100 & 12.13341300 \\ \mathrm{O} & \mathrm{o} & 33.93971400 & 18.17139000 & 12.24661200 \\ \mathrm{O} & \mathrm{o} & 34.75157900 & 16.45030600 & 10.41522000 \\ \mathrm{O} & \mathrm{o} & 32.13603800 & 14.12958900 & 14.11446000 \\ \mathrm{O} & \mathrm{o} & 28.52899200 & 20.17533800 & 16.43829900 \\ \mathrm{O} & \mathrm{o} & 32.25436500 & 16.32334600 & 16.40213700 \\ \mathrm{O} & \mathrm{o} & 32.35334400 & 22.43892200 & 13.91670000 \\ \mathrm{O} & \mathrm{o} & 28.65288200 & 16.47274300 & 20.08807300 \\ \mathrm{O} & \mathrm{o} & 34.66232600 & 20.13305100 & 13.90602400\end{array}$




\begin{tabular}{|c|c|c|}
\hline $\mathrm{Si}$ & o $\quad 31.68572300$ & $15.27415200 \quad 13.05350100$ \\
\hline $\mathrm{Si}$ & o 29.51043300 & $19.67799000 \quad 15.24730600$ \\
\hline $\mathrm{Si}$ & o 33.48779300 & $13.47159300 \quad 19.17435000$ \\
\hline $\mathrm{Si}$ & o $\quad 27.42154900$ & $19.57698600 \quad 13.08447700$ \\
\hline $\mathrm{Al}$ & o $\quad 31.57017800$ & $17.36539500 \quad 15.23387700$ \\
\hline $\mathrm{Si}$ & o $\quad 29.53701700$ & $15.18591900 \quad 10.89309800$ \\
\hline $\mathrm{Si}$ & o 33.53184800 & $23.47901300 \quad 13.44129000$ \\
\hline $\mathrm{Si}$ & o 31.74678900 & $15.19515600 \quad 17.41418200$ \\
\hline $\mathrm{Si}$ & o $\quad 29.17510000$ & $21.33459100 \quad 11.28180100$ \\
\hline $\mathrm{Si}$ & o 31.32740100 & $16.97223900 \quad 9.10742800$ \\
\hline $\mathrm{Si}$ & o 33.48294000 & $19.14939300 \quad 13.43319900$ \\
\hline $\mathrm{Si}$ & o 35.66904000 & $21.32790300 \quad 13.43906400$ \\
\hline $\mathrm{Si}$ & o 31.70993200 & $13.06555300 \quad 15.25967800$ \\
\hline $\mathrm{Si}$ & o 29.56456900 & $15.22626200 \quad 19.55439400$ \\
\hline $\mathrm{Si}$ & o $\quad 27.36185900$ & $19.55984500 \quad 17.39102500$ \\
\hline $\mathrm{Si}$ & o 33.48233900 & $16.90735000 \quad 11.29809900$ \\
\hline $\mathrm{Si}$ & o 31.28321900 & $21.30611200 \quad 13.47463300$ \\
\hline $\mathrm{Si}$ & o 37.41072400 & $23.05422500 \quad 15.22890500$ \\
\hline $\mathrm{Si}$ & o 33.48004000 & $11.27368500 \quad 16.93852500$ \\
\hline $\mathrm{Si}$ & o $\quad 27 \cdot 38610300$ & $17 \cdot 35510800 \quad 10.88450800$ \\
\hline $\mathrm{Si}$ & o $\quad 25.21274600$ & $19.53996900 \quad 15.23290800$ \\
\hline $\mathrm{Si}$ & o $\quad 27.38930300$ & $13.05907600 \quad 19.54203300$ \\
\hline $\mathrm{Si}$ & o $\quad 29.14716500$ & $19.13498800 \quad 9.09538600$ \\
\hline $\mathrm{Si}$ & o 37.82183600 & $21.29405700 \quad 11.27180300$ \\
\hline $\mathrm{Si}$ & о 27.38844000 & $17 \cdot 37164600 \quad 19 \cdot 55891400$ \\
\hline $\mathrm{Si}$ & o 29.54003900 & $10.88670500 \quad 15.20858000$ \\
\hline $\mathrm{Si}$ & o 35.64199600 & $16.96376800 \quad 9.12388900$ \\
\hline $\mathrm{H}$ & $-1 \quad 32.39005900$ & $13.02070900 \quad 20.02956400$ \\
\hline $\mathrm{O}$ & o 33.94056000 & $\begin{array}{lll} & 12.27132200 & 18.15507700\end{array}$ \\
\hline $\mathrm{H}$ & $-1 \quad 34.62965400$ & $13.87839300 \quad 19.95766200$ \\
\hline $\mathrm{O}$ & o 26.15163900 & $20.04535100 \quad 13.97871700$ \\
\hline $\mathrm{H}$ & $\begin{array}{ll}-1 & 28.73210000\end{array}$ & $14.06028900 \quad 10.46662200$ \\
\hline $\mathrm{H}$ & $-1 \quad 33.93653800$ & $24.26800200 \quad 14.60115800$ \\
\hline $\mathrm{H}$ & $-1 \quad 33.07615400$ & $24.34377700 \quad 12.36014300$ \\
\hline $\mathrm{H}$ & $\begin{array}{ll}-1 & 28.72535900\end{array}$ & $22.45719700 \quad 10.47337000$ \\
\hline $\mathrm{O}$ & o 30.42403100 & $18.23962000 \quad 8.62132100$ \\
\hline $\mathrm{H}$ & $-1 \quad 32.13043200$ & $16.55284200 \quad 7.97406000$ \\
\hline $\mathrm{H}$ & -130.35425900 & $14.75342600 \quad 20.68737700$ \\
\hline $\mathrm{H}$ & $\begin{array}{ll}-1 & 26.92599300\end{array}$ & $20.68738700 \quad 18.18171900$ \\
\hline $\mathrm{H}$ & $-1 \quad 38.28816100$ & $23.49674800 \quad 14.13130700$ \\
\hline $\mathrm{H}$ & -136.51871300 & $24.15734400 \quad 15.61383800$ \\
\hline $\mathrm{H}$ & $-1 \quad 38.21669800$ & $22.63909600 \quad 16.37097300$ \\
\hline $\mathrm{H}$ & $-1 \quad 32.39006600$ & $10.40172000 \quad 17.41052500$ \\
\hline $\mathrm{H}$ & $-1 \quad 34.62966700$ & $10.47355200 \quad 16.55288000$ \\
\hline $\mathrm{O}$ & o 27.90963500 & $18.17210000 \quad 9.56060500$ \\
\hline $\mathrm{H}$ & $-1 \quad 26.23281300$ & $16.55955800 \quad 10.46660800$ \\
\hline $\mathrm{H}$ & $-1 \quad 24.34624200$ & $18.44639500 \quad 14.81744100$ \\
\hline $\mathrm{H}$ & $\begin{array}{ll}-1 & 24.42202800\end{array}$ & $20.68741100 \quad 15.67774900$ \\
\hline $\mathrm{H}$ & $\begin{array}{ll}-1 & 27.85029100\end{array}$ & $12.24950000 \quad 20.68740400$ \\
\hline $\mathrm{H}$ & $\begin{array}{ll}-1 \quad 26.98989600 \\
\end{array}$ & $12.17370500 \quad 18.44639800$ \\
\hline $\mathrm{H}$ & $-1 \quad 26.23277700$ & $13.87170000 \quad 19.96464600$ \\
\hline 11 & $\begin{array}{ll}-1 & 28.72534500\end{array}$ & $19.95792700 \quad 7.97404700$ \\
\hline
\end{tabular}




$\begin{array}{lllll}\mathrm{H} & -1 & 38.94616100 & 21.76404000 & 12.09551000 \\ \mathrm{H} & -1 & 37.36572600 & 22.38619000 & 10.40183900 \\ \mathrm{H} & -1 & 38.22340400 & 20.14651800 & 10.47330000 \\ \mathrm{H} & -1 & 26.92598000 & 18.18174600 & 20.68738100 \\ \mathrm{H} & -1 & 26.30380600 & 16.48807700 & 19.10700700 \\ \mathrm{H} & -1 & 30.35424900 & 9.74386400 & 15.67781000 \\ \mathrm{H} & -1 & 28.66054000 & 11.32425900 & 16.29993900 \\ \mathrm{H} & -1 & 28.73209900 & 10.46661500 & 14.06029600 \\ \mathrm{H} & -1 & 36.44050900 & 15.83009000 & 8.66720200 \\ \mathrm{H} & -1 & 34.74683400 & 17.41051600 & 8.04507400 \\ \mathrm{H} & -1 & 36.51628500 & 18.07110400 & 9.52758900 \\ \mathrm{H} & \mathrm{o} & 33.94771600 & 17.93569000 & 15.37563400 \\ \mathrm{O} & \mathrm{o} & 33.20693800 & 18.21551000 & 14.81840400\end{array}$

$\mathrm{R}^{*}(\mathrm{H}-\mathrm{FAU})$

(Electronic Energy: -10882.8471689 Ha):

\begin{tabular}{|c|c|c|}
\hline 0 & o -1.46363400 & $0.37411600-0.00009400$ \\
\hline $\mathrm{O}$ & o -4.98565400 & $-9.20881100 \quad 0.88299100$ \\
\hline $\mathrm{O}$ & o -4.87782800 & $-5.52911000-2.34027400$ \\
\hline $\mathrm{O}$ & o $\quad-7.81559300$ & $-0.25436200 \quad-0.09455900$ \\
\hline $\mathrm{O}$ & o -4.97705700 & $-6.58079700 \quad 3.57591400$ \\
\hline $\mathrm{O}$ & o -10.02013100 & $-0.46467900 \quad-2.45899500$ \\
\hline $\mathrm{O}$ & o -7.74911800 & $-5.61442800 \quad-5.30558200$ \\
\hline $\mathrm{O}$ & o $\quad-7.01818900$ & $-4.86061900-0.89572400$ \\
\hline $\mathrm{O}$ & o $\quad-9.35443400$ & $-2.34466400 \quad-0.70098000$ \\
\hline $\mathrm{O}$ & o $\quad-9.38069100$ & $-4.97035600 \quad-3.29358900$ \\
\hline $\mathrm{O}$ & $0 \quad-3.18421200$ & $1.23194700 \quad-1.84999700$ \\
\hline $\mathrm{O}$ & o -11.83640500 & $-2.38377800 \quad 1.71228800$ \\
\hline $\mathrm{O}$ & o $\quad-6.75825500$ & $-7.47516900 \quad 1.81174100$ \\
\hline $\mathrm{O}$ & o $\quad-9.38513300$ & $-7.33592300 \quad 4.24729200$ \\
\hline $\mathrm{O}$ & o $\quad-8.38766500$ & $-1.30854900-4.37766600$ \\
\hline $\mathrm{O}$ & o $\quad-5.82573800$ & $-4.04781100 \quad-4.34998000$ \\
\hline $\mathrm{O}$ & o $\quad-5.92332000$ & $-1.44883500-1.53044700$ \\
\hline $\mathrm{O}$ & o $\quad-7.27988600$ & $-6.61393100 \quad-2.86950700$ \\
\hline $\mathrm{O}$ & o $\quad-7.31031200$ & $-2.63422800 \quad 0.98735000$ \\
\hline $\mathrm{O}$ & o -11.00526500 & $-2.95343400 \quad-2.69961500$ \\
\hline $\mathrm{O}$ & o $\quad-1.42524900$ & $-0.59229700 \quad-2.52714800$ \\
\hline $\mathrm{O}$ & o -10.09753800 & $-3.02579400 \quad 3.58574000$ \\
\hline $\mathrm{O}$ & o $\quad-7.48043500$ & $-5.64351800 \quad 3.58331900$ \\
\hline $\mathrm{O}$ & o $\quad-7.49343600$ & $-9.23065200-0.03815600$ \\
\hline $\mathrm{O}$ & o -7.46585100 & $0.43129800 \quad-2.63493200$ \\
\hline $\mathrm{O}$ & $0-4.08045800$ & $\begin{array}{lll}0.99253100 & -2.70199900\end{array}$ \\
\hline $\mathrm{O}$ & o -3.27028600 & $-4.90829700 \quad-4.32856600$ \\
\hline $\mathrm{O}$ & o $\quad-5.84045400$ & $-7.29482900 \quad-0.72321400$ \\
\hline $\mathrm{O}$ & o -9.43274800 & $-1.20485800 \quad 1.71103700$ \\
\hline $\mathrm{O}$ & o $\quad-5.74240900$ & $-4.98920800 \quad 1.67818300$ \\
\hline $\mathrm{O}$ & o $\quad-5.65665300$ & $1.10511700-0.81436200$ \\
\hline $\mathrm{O}$ & o $\quad-9.33815300$ & $-4.89377100 \quad 5.34241300$ \\
\hline $\mathrm{O}$ & o $\quad-3.33346800$ & $-1.23761600 \quad-0.82447400$ \\
\hline $\mathrm{Si}$ & o $\quad-6.30175200$ & $-6.06061400-1.68550400$ \\
\hline $\mathrm{Si}$ & o -8.45964900 & $-1.64828900 \quad 0.49217900$ \\
\hline $\mathrm{Si}$ & o -4.50167700 & $-7.89581000 \quad 4.42373800$ \\
\hline
\end{tabular}




\begin{tabular}{|c|c|c|c|}
\hline $\mathrm{Si}$ & o -10.55084400 & -1.78657400 & -1.65734100 \\
\hline $\mathrm{Al}$ & o $\quad-6.41516600$ & -3.98335800 & 0.46578200 \\
\hline $\mathrm{Si}$ & o $\quad-8.44156300$ & -6.16177200 & -3.91949500 \\
\hline $\mathrm{Si}$ & o $\quad-4.46124400$ & 2.11849400 & -1.30111400 \\
\hline $\mathrm{Si}$ & o $\quad-6.24859700$ & -6.14990000 & 2.64704300 \\
\hline $\mathrm{Si}$ & o $\quad-8.80696500$ & -0.02348700 & o $\quad-3.46130800$ \\
\hline $\mathrm{Si}$ & o $\quad-6.71302800$ & -4.40875700 & -5.67785100 \\
\hline $\mathrm{Si}$ & o $\quad-4.50353900$ & -2.20036000 & -1.36204000 \\
\hline $\mathrm{Si}$ & $\begin{array}{ll}0 & -2.32700300\end{array}$ & -0.04883500 & -1.30356700 \\
\hline $\mathrm{Si}$ & o $\quad-6.27820300$ & -8.29368000 & 0.47963800 \\
\hline $\mathrm{Si}$ & o $\quad-8.42654200$ & -6.14019100 & 4.81061800 \\
\hline $\mathrm{Si}$ & o -10.62010400 & -1.80334100 & 2.64631500 \\
\hline $\mathrm{Si}$ & o $\quad-4.52327600$ & -4.39018600 & -3.44280200 \\
\hline $\mathrm{Si}$ & o $\quad-6.71998500$ & -0.02899100 & -1.26207700 \\
\hline $\mathrm{Si}$ & o $\quad-0.58184300$ & 1.68816000 & 0.48051900 \\
\hline $\mathrm{Si}$ & o $\quad-4.50923300$ & -10.08716200 & 2.19332600 \\
\hline $\mathrm{Si}$ & o -10.58718100 & -4.01719200 & -3.86547100 \\
\hline $\mathrm{Si}$ & o -12.77336100 & -1.82261400 & 0.48368100 \\
\hline $\mathrm{Si}$ & o - -10.60167900 & -8.30485500 & 4.79698600 \\
\hline $\mathrm{Si}$ & o $\quad-8.83576800$ & -2.22454300 & -5.65369600 \\
\hline $\mathrm{Si}$ & o $\quad-0.17671300$ & -0.06764100 & -3.46949100 \\
\hline $\mathrm{Si}$ & o -10.60222700 & -3.99267300 & 4.81472700 \\
\hline $\mathrm{Si}$ & o $\quad-8.45089400$ & -10.47927400 & o $\quad 0.46300500$ \\
\hline S1 & $\begin{array}{ll}\text { o } & -2.35703600\end{array}$ & -4.40112700 & -5.61521400 \\
\hline $\mathrm{H}$ & $\begin{array}{ll}-1 & -5.59982400\end{array}$ & -8.34224300 & 5.28399600 \\
\hline $\mathrm{O}$ & o $\quad-4.03854100$ & -9.10567700 & 3.41970000 \\
\hline $\mathrm{H}$ & $-1 \quad-3.36023200$ & -7.48457300 & 5.21211400 \\
\hline $\mathrm{O}$ & o -11.83431200 & -1.32816000 & -0.77480000 \\
\hline $\mathrm{H}$ & $-1 \quad-9.25769100$ & $-7 \cdot 30263900$ & -4.27890000 \\
\hline $\mathrm{H}$ & $-1 \quad-4.05334000$ & 2.90501500 & -0.14437700 \\
\hline $\mathrm{H}$ & $-1 \quad-4.91371700$ & 2.98079500 & -2.38539000 \\
\hline $\mathrm{H}$ & $\begin{array}{ll}-1 & -9.26455300\end{array}$ & 1.09425700 & -4.27218200 \\
\hline $\mathrm{O}$ & o $\quad-7.53474400$ & -3.08024200 & o -6.14114500 \\
\hline $\mathrm{H}$ & $\begin{array}{ll}-1 & -5.85949000\end{array}$ & -4.81015900 & -6.77145100 \\
\hline $\mathrm{H}$ & $\begin{array}{ll}-1 & -7.63561600\end{array}$ & -6.60953700 & 5.94181900 \\
\hline $\mathrm{H}$ & $-1-11.06388100$ & -0.67559600 & 3.43618100 \\
\hline $\mathrm{H}$ & $\begin{array}{ll}-1 & 0.29826200\end{array}$ & 2.13375200 & -0.61422600 \\
\hline $\mathrm{H}$ & $\begin{array}{ll}-1 & -1.47117400\end{array}$ & 2.79433400 & 0.86828900 \\
\hline $\mathrm{H}$ & $\begin{array}{ll}-1 & 0.22680800\end{array}$ & 1.27611200 & 1.62542800 \\
\hline $\mathrm{H}$ & $\begin{array}{ll}-1 & -5.59981300\end{array}$ & -10.96122200 & 2.66496200 \\
\hline $\mathrm{H}$ & $-1-3.36021300$ & -10.88941500 & 1.80733600 \\
\hline $\mathrm{O}$ & o -10.05205800 & -3.21923300 & -5.19771300 \\
\hline $\mathrm{H}$ & $-1-11.75708200$ & -4.80340800 & -4.27893400 \\
\hline $\mathrm{H}$ & $\begin{array}{ll}-1 & -13.64365000\end{array}$ & -2.91657900 & 0.07190600 \\
\hline $\mathrm{H}$ & $-1-13.56786700$ & -0.67556100 & 0.93221600 \\
\hline $\mathrm{H}$ & $-1-10.13959000$ & -9.11347000 & 5.94186600 \\
\hline $\mathrm{H}$ & $-1-10.99998700$ & -9.18926800 & o $\quad 3.70086200$ \\
\hline $\mathrm{H}$ & $-1-11.75710200$ & -7.49127300 & 5.21910800 \\
\hline $\mathrm{H}$ & $-1 \quad-9.26454800$ & -1.40504100 & -6.77148900 \\
\hline $\mathrm{H}$ & $-1 \quad 0.95626500$ & 0.40105300 & -2.65003200 \\
\hline $\mathrm{H}$ & $-1 \quad-0.62417400$ & 1.02320800 & -4.34368000 \\
\hline $\mathrm{H}$ & $\begin{array}{ll}-1 & 0.23351200\end{array}$ & -1.21645500 & -4.27224300 \\
\hline
\end{tabular}




$\begin{array}{lllll}\mathrm{H} & -1 & -11.06389700 & -3.18123000 & 5.94184000 \\ \mathrm{H} & -1 & -11.68608000 & -4.87489800 & 4.36147100 \\ \mathrm{H} & -1 & -7.63562800 & -11.61909600 & 0.93226300 \\ \mathrm{H} & -1 & -9.32932400 & -10.03870600 & 1.55439100 \\ \mathrm{H} & -1 & -9.25778000 & -10.89635200 & -0.68523900 \\ \mathrm{H} & -1 & -1.54937800 & -5.53287500 & -6.07827500 \\ \mathrm{H} & -1 & -3.24304200 & -3.95249600 & -6.70041700 \\ \mathrm{H} & -1 & -1.47362300 & -3.29187200 & -5.21792700 \\ \mathrm{H} & \mathrm{o} & -4.01991100 & -3.91584400 & 0.14591000 \\ \mathrm{O} & \mathrm{o} & -4.75871700 & -3.32217200 & -0.13315100 \\ \mathrm{C} & \mathrm{O} & -3.05230500 & -5.88007500 & 0.58532400 \\ \mathrm{C} & \mathrm{o} & -2.14512700 & -4.92537900 & 0.31813400 \\ \mathrm{H} & \mathrm{o} & -3.33114700 & -6.12285700 & 1.60573600 \\ \mathrm{H} & \mathrm{o} & -3.53663300 & -6.43783000 & -0.21483100 \\ \mathrm{C} & \mathrm{o} & -1.74716500 & -4.56674600 & -1.04093300 \\ \mathrm{H} & \mathrm{o} & -1.67305100 & -4.40189900 & 1.14674700 \\ \mathrm{H} & \mathrm{o} & -2.20243700 & -5.15579700 & -1.83634200 \\ \mathrm{C} & \mathrm{O} & -0.85714100 & -3.61142000 & -1.37320400 \\ \mathrm{C} & \mathrm{o} & -0.13134800 & -2.74974900 & -0.44438300 \\ \mathrm{H} & \mathrm{o} & -0.63139300 & -3.46309400 & -2.42856800 \\ \mathrm{C} & \mathrm{o} & 0.94148100 & -2.04090900 & -0.80929800 \\ \mathrm{H} & \mathrm{o} & -0.48082900 & -2.68567100 & 0.58339300 \\ \mathrm{H} & \mathrm{o} & 1.47832800 & -1.41079600 & -0.10818900 \\ \mathrm{H} & \mathrm{o} & 1.33013600 & -2.09544400 & -1.82374700 \\ & & & & \end{array}$

A* (H-FAU)

(Electronic Energy: -10882.8447529 Ha):

$\begin{array}{lllll}\mathrm{O} & \mathrm{o} & -1.80054000 & 1.23067000 & 0.00092500 \\ \mathrm{O} & \mathrm{o} & -5.31879900 & -8.30887300 & 0.97120300 \\ \mathrm{O} & \mathrm{o} & -5.26398100 & -4.62803600 & -2.37955000 \\ \mathrm{O} & \mathrm{o} & -7.99729300 & 0.47026400 & -0.04656800 \\ \mathrm{O} & \mathrm{o} & -5.32706100 & -5.80487400 & 3.53651000 \\ \mathrm{O} & \mathrm{o} & -10.38828100 & 0.34954400 & -2.51366700 \\ \mathrm{O} & \mathrm{o} & -8.04229600 & -4.75905700 & -5.27991500 \\ \mathrm{O} & \mathrm{o} & -7.29392900 & -4.32699600 & -0.62830500 \\ \mathrm{O} & \mathrm{o} & -9.67017300 & -1.49198300 & -0.72584800 \\ \mathrm{O} & \mathrm{o} & -9.71287600 & -4.10995200 & -3.29972600 \\ \mathrm{O} & \mathrm{o} & -3.49015000 & 2.11518200 & -1.86387800 \\ \mathrm{O} & \mathrm{o} & -12.16856000 & -1.53118600 & 1.71860600 \\ \mathrm{O} & \mathrm{o} & -7.04037900 & -6.38237800 & 1.55034200 \\ \mathrm{O} & \mathrm{o} & -9.70724400 & -6.49638500 & 4.24353100 \\ \mathrm{O} & \mathrm{o} & -8.66817500 & -0.42238600 & -4.38787500 \\ \mathrm{O} & \mathrm{o} & -6.13875100 & -3.12204600 & -4.36608000 \\ \mathrm{O} & \mathrm{o} & -6.19922700 & -0.53538100 & -1.74983900 \\ \mathrm{O} & \mathrm{o} & -7.64838700 & -5.76710900 & -2.83556100 \\ \mathrm{O} & \mathrm{o} & -7.74015300 & -1.97200400 & 1.04623400 \\ \mathrm{O} & \mathrm{o} & -11.37786200 & -2.14507800 & -2.65258300 \\ \mathrm{O} & \mathrm{o} & -1.72809000 & 0.25845900 & -2.49709300 \\ \mathrm{O} & \mathrm{o} & -10.41815900 & -2.16067300 & 3.58464800 \\ \mathrm{O} & \mathrm{o} & -7.81102200 & -4.81731700 & 3.56563400 \\ \mathrm{O} & \mathrm{o} & -7.80796400 & -8.37566000 & -0.00490500 \\ \mathrm{O} & \mathrm{o} & -7.87748400 & 1.34003100 & -2.56247300 \\ & & & & \end{array}$




\begin{tabular}{|c|c|c|}
\hline $\mathrm{O}$ & o -4.32336600 & $-2.12704700 \quad-2.73011300$ \\
\hline $\mathrm{O}$ & o -3.62678700 & $-4.08253700-4.36722900$ \\
\hline $\mathrm{O}$ & o -6.03003500 & $-6.64056900-0.92672200$ \\
\hline $\mathrm{O}$ & o $\quad-9.74667400$ & $-0.36638100 \quad 1.69504600$ \\
\hline $\mathrm{O}$ & o $\quad-5.94237900$ & $-3.93175000 \quad 1.89603400$ \\
\hline $\mathrm{O}$ & o $\quad-5.97846700$ & $1.96554300-0.81774500$ \\
\hline $\mathrm{O}$ & o -9.66206600 & $0-4.03301100 \quad 5.32054100$ \\
\hline $\mathrm{O}$ & o $\quad-3.69615700$ & $-0.34891100 \quad-0.83773800$ \\
\hline $\mathrm{Si}$ & o $\quad-6.58325600$ & $-5.29935300 \quad-1.69473300$ \\
\hline $\mathrm{Si}$ & o $\quad-8.76331600$ & $\begin{array}{ll}-0.87016900 & 0.49743500\end{array}$ \\
\hline $\mathrm{Si}$ & o -4.81487800 & $-7.08697000 \quad 4.43269600$ \\
\hline $\mathrm{Si}$ & o -10.88863700 & $-0.94717400 \quad-1.66273600$ \\
\hline $\mathrm{Al}$ & o $\quad-6.63427600$ & $-3.09237000 \quad 0.36535600$ \\
\hline $\mathrm{Si}$ & o $\quad-8.77128300$ & $-5.30478200 \quad-3.91704500$ \\
\hline $\mathrm{Si}$ & o -4.78136100 & $2.97446600 \quad-1.30887000$ \\
\hline $\mathrm{Si}$ & o -6.58000500 & $-5.30114700 \quad 2.64462700$ \\
\hline $\mathrm{Si}$ & o -9.14451900 & $0.83711700 \quad-3.46010600$ \\
\hline $\mathrm{Si}$ & o -7.02554900 & $-3.53739900-5.67199200$ \\
\hline $\mathrm{Si}$ & o -4.83658100 & $-1.37379800 \quad-1.37273800$ \\
\hline $\mathrm{Si}$ & o -2.67198500 & $0.80999700-1.30553200$ \\
\hline $\mathrm{Si}$ & o -6.55305200 & $\begin{array}{ll}-7.43490400 & 0.37458700\end{array}$ \\
\hline $\mathrm{Si}$ & o -8.77044600 & $-5.29239000 \quad 4.81059300$ \\
\hline $\mathrm{Si}$ & o -10.94258000 & $-0.94158200 \quad 2.63188700$ \\
\hline $\mathrm{Si}$ & o $\quad-4.83239500$ & $-3.49426500 \quad-3.45895200$ \\
\hline $\mathrm{Si}$ & o $\quad-7.00609400$ & $0.80523500 \quad-1.29168900$ \\
\hline $\mathrm{Si}$ & o $\quad-0.91416900$ & $2.54017900 \quad 0.47531400$ \\
\hline $\mathrm{Si}$ & o -4.82776300 & $\begin{array}{ll}-9.24784500 \quad 2.21777500\end{array}$ \\
\hline $\mathrm{Si}$ & o -10.92688800 & $-3.15692700 \quad-3.85342000$ \\
\hline $\mathrm{Si}$ & o -13.10168800 & $-0.96781100 \quad 0.48400500$ \\
\hline $\mathrm{Si}$ & o -10.93704300 & $-7.45594000 \quad 4.79544800$ \\
\hline $\mathrm{Si}$ & o -9.15703300 & $-1.34945700 \quad-5.64363300$ \\
\hline $\mathrm{Si}$ & $0 \quad-0.50517200$ & $0.78431100 \quad-3.47031800$ \\
\hline $\mathrm{Si}$ & o -10.93611700 & $-3.12622100 \quad 4.80225300$ \\
\hline $\mathrm{Si}$ & o $\quad-8.77951400$ & $-9.62512300 \quad 0.46144900$ \\
\hline $\mathrm{Si}$ & o -2.68724900 & $-3.54730300 \quad-5.61930300$ \\
\hline $\mathrm{H}$ & $-1 \quad-5.92889300$ & $-7.48692600 \quad 5.28071800$ \\
\hline $\mathrm{O}$ & o -4.33504200 & $-8.31776800 \quad 3.48028500$ \\
\hline $\mathrm{H}$ & $-1-3.68929800$ & $-6.62924200 \quad 5.20881600$ \\
\hline $\mathrm{O}$ & o -12.14965200 & $-0.46667500 \quad-0.75761200$ \\
\hline $\mathrm{H}$ & $-1 \quad-9.58685200$ & $-6.44734600 \quad-4.28222400$ \\
\hline $\mathrm{H}$ & $-1-4.38241400$ & $3.76036700-0.14768800$ \\
\hline $\mathrm{H}$ & $-1 \quad-5.24279800$ & $3.83614200 \quad-2.38870300$ \\
\hline $\mathrm{H}$ & $-1-9.59359300$ & $1.94956200 \quad-4.27547600$ \\
\hline $\mathrm{O}$ & o $\quad-7.89115200$ & $-2.24072500-6.15094600$ \\
\hline $\mathrm{H}$ & $-1-6.18852000$ & $-3.95479300 \quad-6.77478600$ \\
\hline $\mathrm{H}$ & $-1 \quad-7.96469300$ & $-5.75420900 \quad 5.93853100$ \\
\hline $\mathrm{H}$ & $-1-11.39295900$ & $0.17975200 \quad 3.43287300$ \\
\hline $\mathrm{H}$ & $-1-0.03079100$ & $2.98911300-0.61753900$ \\
\hline $\mathrm{H}$ & $-1-1.80023900$ & $3.64970900 \quad 0.86499200$ \\
\hline $\mathrm{H}$ & $-1-0.10225400$ & $2.13146100 \quad 1.62212700$ \\
\hline $\mathrm{H}$ & $-1-5.92888600$ & $-10.10591500 \quad 2.66167900$ \\
\hline $\mathrm{H}$ & $-1-3.68928500$ & $-10.03408300 \quad 1.80403400$ \\
\hline
\end{tabular}




\begin{tabular}{lllll}
$\mathrm{O}$ & $\mathrm{o}$ & -10.39279300 & -2.30553400 & -5.14916100 \\
$\mathrm{H}$ & -1 & -12.08613900 & -3.94807700 & -4.28223800 \\
$\mathrm{H}$ & -1 & -13.97271000 & -2.06124000 & 0.06859500 \\
$\mathrm{H}$ & -1 & -13.89692400 & 0.17977600 & 0.92890300 \\
$\mathrm{H}$ & -1 & -10.46866100 & -8.25813500 & 5.93855800 \\
$\mathrm{H}$ & -1 & -11.32905600 & -8.33393000 & 3.69755200 \\
$\mathrm{H}$ & -1 & -12.08617500 & -6.63593500 & 5.21580000 \\
$\mathrm{H}$ & -1 & -9.59360700 & -0.54970800 & -6.77479900 \\
$\mathrm{H}$ & -1 & 0.62720900 & 1.25640500 & -2.65333600 \\
$\mathrm{H}$ & -1 & -0.95322600 & 1.87855500 & -4.34700700 \\
$\mathrm{H}$ & -1 & -0.09554800 & -0.36111700 & -4.27554600 \\
$\mathrm{H}$ & -1 & -11.39297200 & -2.32588900 & 5.93853500 \\
$\mathrm{H}$ & -1 & -12.01514600 & -4.01955800 & 4.35816100 \\
$\mathrm{H}$ & -1 & -7.96470300 & -10.76377100 & 0.92896400 \\
$\mathrm{H}$ & -1 & -9.65841200 & -9.18337600 & 1.55109300 \\
$\mathrm{H}$ & -1 & -9.58685300 & -10.04102000 & -0.68855000 \\
$\mathrm{H}$ & -1 & -1.87844300 & -4.67754500 & -6.08164400 \\
$\mathrm{H}$ & -1 & -3.57211800 & -3.09711900 & -6.70377200 \\
$\mathrm{H}$ & -1 & -1.80266700 & -2.43653100 & -5.22125700 \\
$\mathrm{O}$ & $\mathrm{o}$ & -5.15363000 & -2.43247000 & -0.21451600 \\
$\mathrm{C}$ & $\mathrm{o}$ & -4.98443100 & -3.09965200 & 2.66443900 \\
$\mathrm{C}$ & $\mathrm{o}$ & -5.67830000 & -2.39462400 & 3.81975900 \\
$\mathrm{H}$ & $\mathrm{o}$ & -4.19826200 & -3.77312000 & 3.00716400 \\
$\mathrm{H}$ & $\mathrm{o}$ & -6.51113600 & -1.80429700 & 3.42643400 \\
$\mathrm{C}$ & $\mathrm{o}$ & -4.66944300 & -1.52671700 & 4.52873700 \\
$\mathrm{H}$ & $\mathrm{o}$ & -6.10015600 & -3.13325300 & 4.50888400 \\
$\mathrm{H}$ & $\mathrm{o}$ & -4.65921700 & -0.47183400 & 4.26925000 \\
$\mathrm{C}$ & $\mathrm{o}$ & -3.76761700 & -1.98678200 & 5.40591700 \\
$\mathrm{C}$ & $\mathrm{o}$ & -3.63921100 & -3.38037300 & 5.82957900 \\
$\mathrm{H}$ & $\mathrm{o}$ & -3.06050200 & -1.28770400 & 5.84880700 \\
$\mathrm{C}$ & $\mathrm{o}$ & -2.71113200 & -3.80228800 & 6.69169000 \\
$\mathrm{H}$ & $\mathrm{o}$ & -4.57097000 & -2.39954400 & 1.93989500 \\
$\mathrm{H}$ & $\mathrm{o}$ & -4.33501500 & -4.10229000 & 5.40306700 \\
$\mathrm{H}$ & $\mathrm{o}$ & -1.99480000 & -3.11189900 & 7.12864400 \\
$\mathrm{H}$ & $\mathrm{o}$ & -2.63594400 & -4.84362300 & 6.98503200 \\
& & & & \\
\hline
\end{tabular}

$\mathrm{B}^{*}(\mathrm{H}-\mathrm{FAU})$

(Electronic Energy: -10882.8747155 Ha):

$\begin{array}{lllll}\mathrm{O} & \mathrm{o} & -1.81572400 & 1.23389900 & 0.00852700 \\ \mathrm{O} & \mathrm{o} & -5.31479300 & -8.38962500 & 0.85188100 \\ \mathrm{O} & \mathrm{o} & -5.29630200 & -4.88098000 & -2.56827300 \\ \mathrm{O} & \mathrm{o} & -7.95297000 & 0.44178600 & -0.02100800 \\ \mathrm{O} & \mathrm{o} & -5.33179900 & -5.69224000 & 3.61988300 \\ \mathrm{O} & \mathrm{o} & -10.38208100 & 0.34249000 & -2.51360900 \\ \mathrm{O} & \mathrm{o} & -7.91033700 & -4.78216400 & -5.13293800 \\ \mathrm{O} & \mathrm{o} & -7.19732400 & -3.88410800 & -1.00803500 \\ \mathrm{O} & \mathrm{o} & -9.66203900 & -1.48588500 & -0.71719700 \\ \mathrm{O} & \mathrm{o} & -9.72147800 & -4.11117700 & -3.30083400 \\ \mathrm{O} & \mathrm{o} & -3.47113500 & 2.14187200 & -1.86944900 \\ \mathrm{O} & \mathrm{o} & -12.16879600 & -1.53338800 & 1.72096200 \\ \mathrm{O} & \mathrm{o} & -7.08698700 & -6.74911800 & 1.90952600 \\ \mathrm{O} & \mathrm{o} & -9.72119700 & -6.47850200 & 4.24942100\end{array}$




\begin{tabular}{|c|c|c|c|}
\hline $\mathrm{O}$ & o $\quad-8.65815900$ & -0.41530300 & -4.39905200 \\
\hline $\mathrm{O}$ & o $\quad-6.02986800$ & -3.04003400 & $0 \quad-4.38363000$ \\
\hline $\mathrm{O}$ & o $\quad-6.16081000$ & -0.55684400 & -1.74264500 \\
\hline $\mathrm{O}$ & o $\quad-7.78003800$ & -5.86546200 & o $\quad-2.67403700$ \\
\hline $\mathrm{O}$ & o $\quad-7.75358000$ & -1.97234100 & 1.11483600 \\
\hline $\mathrm{O}$ & o -11.38011300 & -2.14905500 & -2.63914400 \\
\hline $\mathrm{O}$ & $0-1.71065200$ & 0.26632800 & -2.49555400 \\
\hline $\mathrm{O}$ & o -10.41758300 & -2.15779400 & 3.59095600 \\
\hline $\mathrm{O}$ & o $\quad-7.82407000$ & -4.78757900 & o $\quad 3.58148400$ \\
\hline $\mathrm{O}$ & o $\quad-7.82419900$ & -8.37795800 & - -0.04999900 \\
\hline $\mathrm{O}$ & o $\quad-7.86767700$ & 1.31571600 & -2.55285000 \\
\hline $\mathrm{O}$ & o $\quad-4.34752600$ & -2.34139500 & -2.47400400 \\
\hline $\mathrm{O}$ & o $\quad-3.56598700$ & -4.06276000 & o $\quad-4.32459300$ \\
\hline $\mathrm{O}$ & o $\quad-6.19116400$ & -6.37317400 & -0.61856500 \\
\hline $\mathrm{O}$ & o $\quad-9.75931400$ & -0.34586900 & 1.69242000 \\
\hline $\mathrm{O}$ & o $\quad-6.11676600$ & -4.25899600 & 1.61982500 \\
\hline $\mathrm{O}$ & o $\quad-5.95619200$ & 1.93792000 & -0.81602900 \\
\hline $\mathrm{O}$ & o $\quad-9.66768100$ & -4.03279400 & o $\quad 5 \cdot 34728400$ \\
\hline $\mathrm{O}$ & $\begin{array}{ll}\text { o } & -3.67494100\end{array}$ & -0.33719700 & -0.84461400 \\
\hline $\mathrm{Si}$ & o $\quad-6.64084000$ & -5.22459000 & o -1.68872300 \\
\hline $\mathrm{Si}$ & o $\quad-8.76099700$ & -0.89042800 & o $\quad 0.52086200$ \\
\hline $\mathrm{Si}$ & o $\quad-4.83909100$ & -7.03447900 & 4.41657800 \\
\hline $\mathrm{Si}$ & o -10.88337400 & -0.95153100 & -1.65366600 \\
\hline $\mathrm{Al}$ & o $\quad-6.62447800$ & -3.10529900 & 0.43449200 \\
\hline $\mathrm{Si}$ & o $\quad-8.77017900$ & -5.33146500 & -3.84733000 \\
\hline $\mathrm{Si}$ & o $\quad-4.78558400$ & 2.97215500 & -1.31263700 \\
\hline $\mathrm{Si}$ & o $\quad-6.61503500$ & $-5 \cdot 35761500$ & 2.64593300 \\
\hline $\mathrm{Si}$ & o $\quad-9.14169200$ & 0.83050500 & -3.46100900 \\
\hline $\mathrm{Si}$ & o $\quad-6.97425300$ & -3.53545000 & -5.62438000 \\
\hline $\mathrm{Si}$ & o $\quad-4.84869400$ & -1.41165700 & -1.23573800 \\
\hline $\mathrm{Si}$ & o $\quad-2.67328700$ & 0.82181700 & -1.32913100 \\
\hline $\mathrm{Si}$ & o $\quad-6.61140600$ & -7.45555600 & 0.50105700 \\
\hline $\mathrm{Si}$ & о $\quad-8.76425700$ & -5.28461000 & 4.81125000 \\
\hline $\mathrm{Si}$ & o -10.94282800 & -0.94430100 & 2.63622200 \\
\hline $\mathrm{Si}$ & o $\quad-4.82272300$ & -3.58334700 & -3.42063400 \\
\hline $\mathrm{Si}$ & o $\quad-6.99266200$ & 0.76893100 & -1.29237800 \\
\hline $\mathrm{Si}$ & $0 \quad-0.91422700$ & 2.54510100 & 0.47210100 \\
\hline $\mathrm{Si}$ & o $\quad-4.84222700$ & -9.23381100 & 2.17948300 \\
\hline $\mathrm{Si}$ & o -10.93113400 & -3.15702200 & -3.84556400 \\
\hline $\mathrm{Si}$ & o -13.09966700 & -0.96885200 & o $\quad 0.48646000$ \\
\hline $\mathrm{Si}$ & o -10.93425200 & -7.45210900 & 4.79447500 \\
\hline $\mathrm{Si}$ & o -9.15847900 & -1.35910000 & -5.63977300 \\
\hline $\mathrm{Si}$ & o $\quad-0.49477200$ & 0.78758300 & -3.47936100 \\
\hline $\mathrm{Si}$ & o -10.93126200 & -3.13351800 & 4.80840900 \\
\hline $\mathrm{Si}$ & o $\quad-8.77983100$ & -9.62304000 & 0.45771900 \\
\hline $\mathrm{Si}$ & o $\quad-2.68375000$ & -3.54642700 & -5.61564100 \\
\hline $\mathrm{H}$ & $\begin{array}{ll}-1 & -5.92888500\end{array}$ & -7.48689600 & o $\quad 5.28067400$ \\
\hline $\mathrm{O}$ & o $\quad-4.37829900$ & -8.22630400 & o $\quad 3.39368100$ \\
\hline $\mathrm{H}$ & $\begin{array}{ll}-1 & -3.68930900\end{array}$ & -6.62922600 & o $\quad 5.20876600$ \\
\hline $\mathrm{O}$ & o -12.14476600 & -0.46313700 & -0.75234400 \\
\hline $\mathrm{H}$ & $\begin{array}{ll}-1 & -9.58683600\end{array}$ & -6.44734900 & o $\quad-4.28222400$ \\
\hline $\mathrm{H}$ & $-1 \quad-4.38241300$ & 3.76037600 & -0.14768400 \\
\hline
\end{tabular}




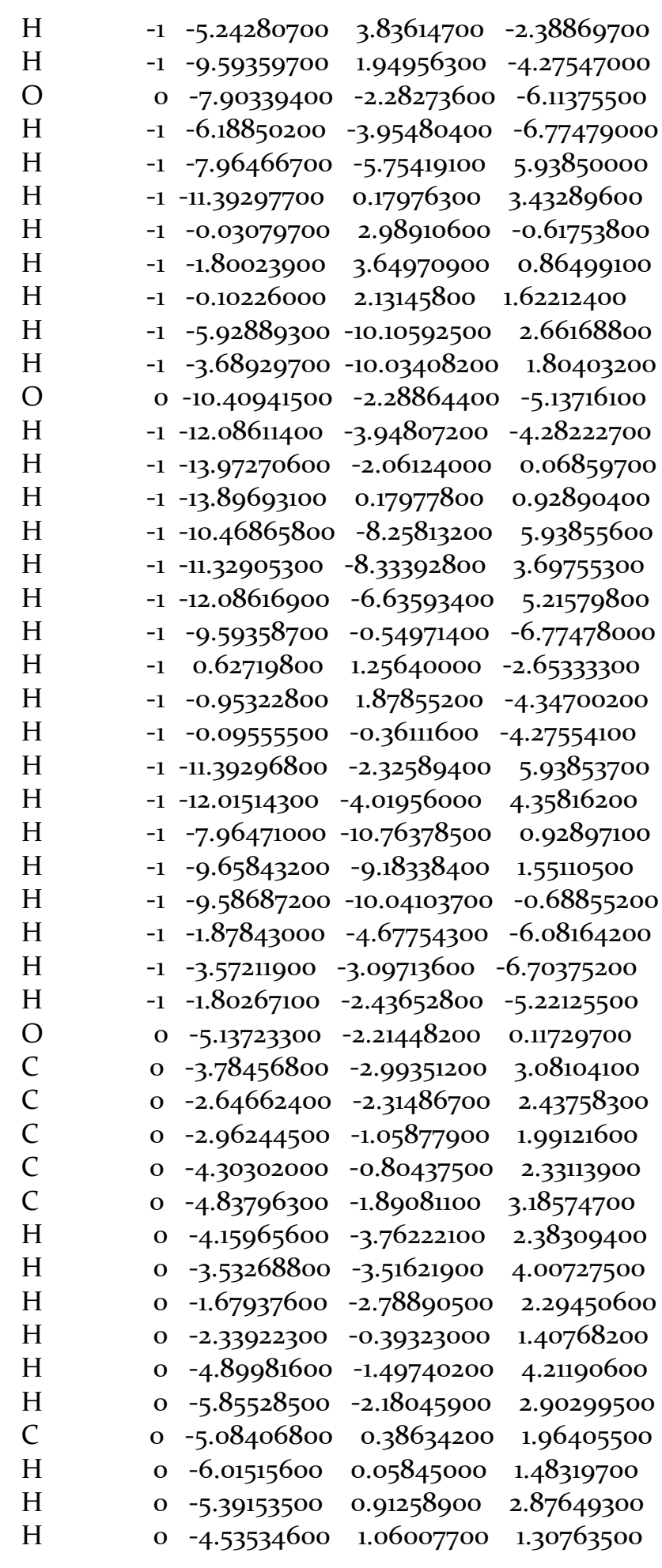

$\mathrm{C}^{*}(\mathrm{H}-\mathrm{FAU})$

(Electronic Energy: -10882.8781001 Ha):
$\mathrm{O}$
o $\quad-1.82215400 \quad 1.22024900 \quad 0.00368200$
$\mathrm{O}$
$\begin{array}{lllll}\text { o } & -5.31395700 & -8.39537900 & 0.83611400\end{array}$
$\mathrm{O}$
$\begin{array}{lllll}\text { o } & -5.32569300 & -5.03897900 & -2.71744800\end{array}$ 


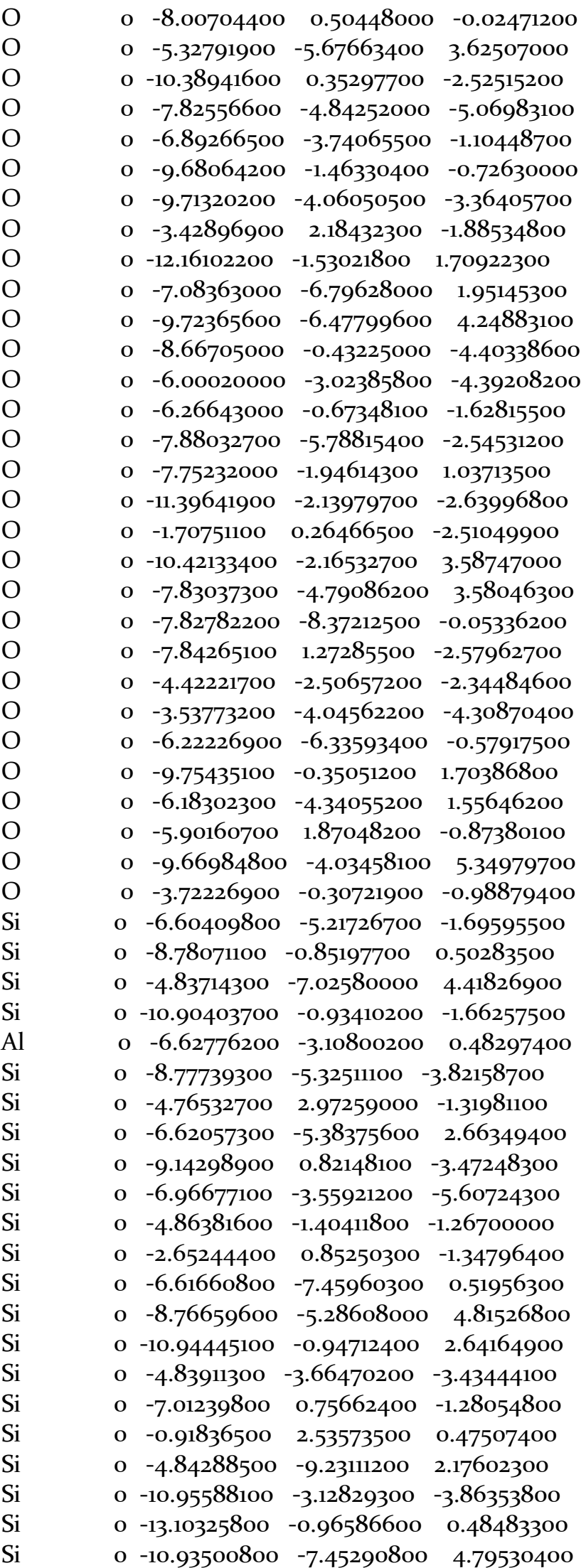




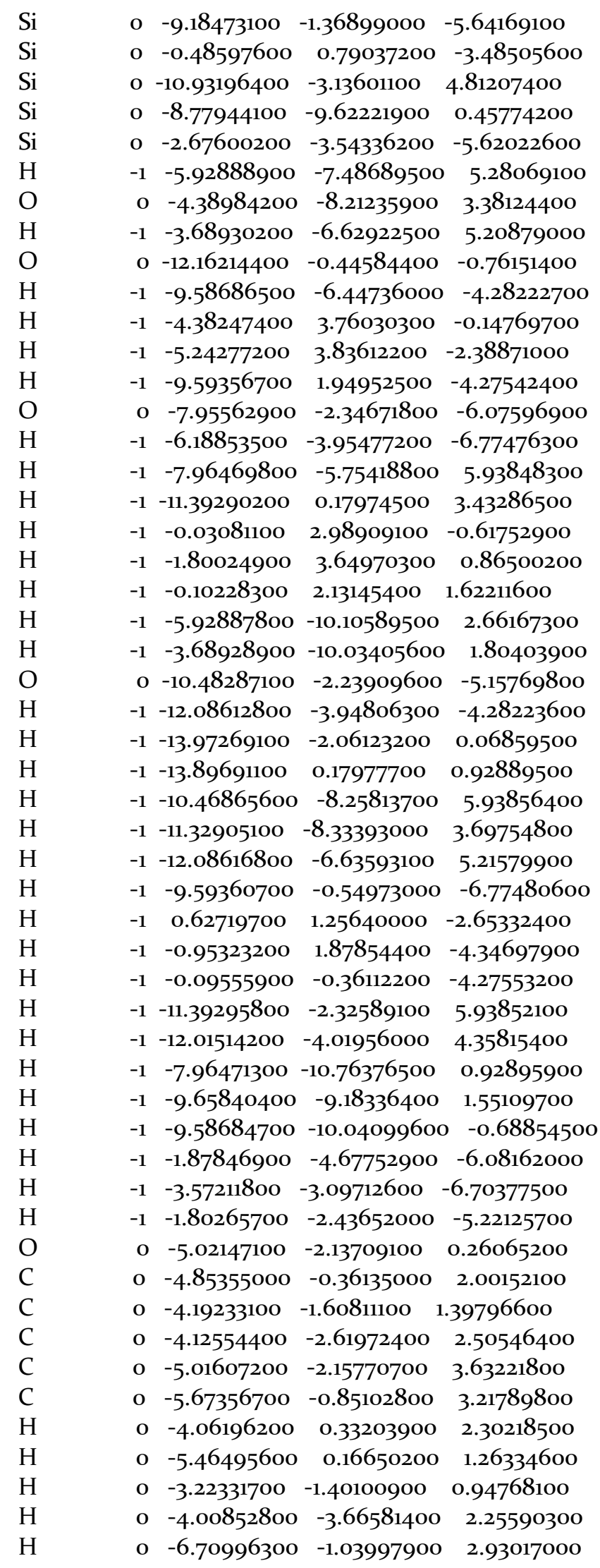




$$
\begin{array}{lllll}
\mathrm{H} & \mathrm{o} & -5.67202400 & -0.10914900 & 4.02190400 \\
\mathrm{C} & \mathrm{o} & -3.52676000 & -2.11364900 & 3.79145000 \\
\mathrm{H} & \mathrm{o} & -3.06671000 & -2.87000600 & 4.41636700 \\
\mathrm{H} & \mathrm{o} & -3.02824400 & -1.14813700 & 3.81178500 \\
\mathrm{H} & \mathrm{o} & -5.58668300 & -2.88189900 & 4.20404300
\end{array}
$$

$\mathrm{F}^{*}$ (H-FAU)

(Electronic Energy: -10882.8821598 Ha):

$\begin{array}{lllll}\mathrm{O} & \mathrm{o} & -1.45751900 & 0.36588700 & 0.00293300 \\ \mathrm{O} & \mathrm{o} & -4.98290400 & -9.20820600 & 0.88850300 \\ \mathrm{O} & \mathrm{o} & -4.94247600 & -5.58592600 & -2.43424100 \\ \mathrm{O} & \mathrm{o} & -7.79157100 & -0.26970000 & -0.08942500 \\ \mathrm{O} & \mathrm{o} & -4.97457400 & -6.57393400 & 3.58761200 \\ \mathrm{O} & \mathrm{o} & -10.02039400 & -0.46804800 & -2.46302200 \\ \mathrm{O} & \mathrm{o} & -7.66316000 & -5.62198200 & -5.21652200 \\ \mathrm{O} & \mathrm{o} & -6.98823500 & -4.82527300 & -0.90939800 \\ \mathrm{O} & \mathrm{o} & -9.35233200 & -2.34208300 & -0.70133200 \\ \mathrm{O} & \mathrm{o} & -9.38700200 & -4.96535600 & -3.29283200 \\ \mathrm{O} & \mathrm{o} & -3.15705900 & 1.26268600 & -1.85813100 \\ \mathrm{O} & \mathrm{o} & -11.83712100 & -2.38499500 & 1.71318000 \\ \mathrm{O} & \mathrm{o} & -6.76058800 & -7.49164700 & 1.84091800 \\ \mathrm{O} & \mathrm{o} & -9.38557100 & -7.33371400 & 4.24727500 \\ \mathrm{O} & \mathrm{o} & -8.38373400 & -1.30907000 & -4.38209700 \\ \mathrm{O} & \mathrm{o} & -5.75791700 & -3.95026600 & -4.38053400 \\ \mathrm{O} & \mathrm{o} & -5.92203800 & -1.47136100 & -1.55041500 \\ \mathrm{O} & \mathrm{o} & -7.36732200 & -6.67290700 & -2.76910100 \\ \mathrm{O} & \mathrm{o} & -7.33104700 & -2.65279900 & 1.01331400 \\ \mathrm{O} & \mathrm{o} & -11.01355500 & -2.95518800 & -2.69129000 \\ \mathrm{O} & \mathrm{o} & -1.40172000 & -0.59638600 & -2.50294200 \\ \mathrm{O} & \mathrm{o} & -10.09624900 & -3.02389500 & 3.58493200 \\ \mathrm{O} & \mathrm{o} & -7.47988300 & -5.63687300 & 3.58685000 \\ \mathrm{O} & \mathrm{o} & -7.48964200 & -9.22668400 & -0.03429200 \\ \mathrm{O} & \mathrm{o} & -7.46539000 & 0.42474800 & -2.63309500 \\ \mathrm{O} & \mathrm{o} & -4.03411700 & -3.04636700 & -2.63732500 \\ \mathrm{O} & \mathrm{o} & -3.24934200 & -4.92052600 & -4.32390900 \\ \mathrm{O} & \mathrm{o} & -5.82808700 & -7.26725900 & -0.67849200 \\ \mathrm{O} & \mathrm{o} & -9.43906300 & -1.19503200 & 1.70640800 \\ \mathrm{O} & \mathrm{o} & -5.73959400 & -5.00677500 & 1.67481600 \\ \mathrm{O} & \mathrm{o} & -5.62991400 & 1.07714200 & -0.83241200 \\ \mathrm{O} & \mathrm{o} & -9.33898300 & -4.89247900 & 5.34405700 \\ \mathrm{O} & \mathrm{o} & -3.34472700 & -1.21235000 & -0.83962000 \\ \mathrm{Si} & \mathrm{o} & -6.32016200 & -6.07053600 & -1.67208400 \\ \mathrm{Si} & \mathrm{o} & -8.45926100 & -1.65408400 & 0.49786600 \\ \mathrm{Si} & \mathrm{o} & -4.50094100 & -7.89435300 & 4.42559400 \\ \mathrm{Si} & \mathrm{o} & -10.55163400 & -1.78622100 & -1.65500700 \\ \mathrm{Al} & \mathrm{o} & -6.40728700 & -3.97914300 & 0.47897100 \\ \mathrm{Si} & \mathrm{o} & -8.44478800 & -6.17258400 & -3.88136200 \\ \mathrm{Si} & \mathrm{o} & -4.45627000 & 2.11989600 & -1.30470700 \\ \mathrm{Si} & \mathrm{o} & -6.24676700 & -6.15484400 & 2.65706900 \\ \mathrm{Si} & \mathrm{o} & -8.80625200 & -0.02667500 & -3.46384300 \\ \mathrm{Si} & \mathrm{o} & -6.68200500 & -4.39420100 & -5.65711300 \\ \mathrm{Si} & \mathrm{o} & -4.50376100 & -2.21593000 & -1.33777800 \\ & & & & \end{array}$




\begin{tabular}{|c|c|c|c|}
\hline $\mathrm{Si}$ & o $\quad-2.32878100$ & -0.02930800 & -1.30609500 \\
\hline $\mathrm{Si}$ & o $\quad-6.27805000$ & -8.29161400 & 0.49736000 \\
\hline $\mathrm{Si}$ & o $\quad-8.42662600$ & -6.13838300 & 4.81150200 \\
\hline $\mathrm{Si}$ & o -10.62034500 & -1.80195200 & 2.64529600 \\
\hline $\mathrm{Si}$ & o -4.50450700 & -4.39946900 & o -3.44383100 \\
\hline $\mathrm{Si}$ & o $\quad-6.70985500$ & -0.04824400 & o $\quad-1.27040800$ \\
\hline $\mathrm{Si}$ & o $\quad-0.58046400$ & 1.68883700 & 0.48248100 \\
\hline $\mathrm{Si}$ & o -4.50788200 & -10.08792000 & o $\quad 2.19768300$ \\
\hline $\mathrm{Si}$ & o -10.59379200 & -4.01364300 & -3.86146400 \\
\hline $\mathrm{Si}$ & o -12.77327400 & -1.82246600 & 0.48457400 \\
\hline $\mathrm{Si}$ & o -10.60106700 & -8.30421100 & 4.79668200 \\
\hline $\mathrm{Si}$ & o $\quad-8.84121400$ & -2.22835200 & -5.65337900 \\
\hline $\mathrm{Si}$ & o $\quad-0.16994500$ & -0.06724900 & -3.47254900 \\
\hline $\mathrm{Si}$ & o -10.60195400 & -3.99161700 & 4.81377700 \\
\hline $\mathrm{Si}$ & o $\quad-8.44912800$ & -10.47694300 & 0.46258300 \\
\hline $\mathrm{Si}$ & o $\quad-2.35437800$ & -4.40106700 & -5.61597600 \\
\hline $\mathrm{H}$ & $\begin{array}{ll}-1 & -5.59981200\end{array}$ & -8.34222500 & 5.28394700 \\
\hline $\mathrm{O}$ & o $\quad-4.03922400$ & -9.10732000 & 3.42437900 \\
\hline $\mathrm{H}$ & $\begin{array}{ll}-1 & -3.36023400\end{array}$ & -7.48457200 & 5.21210200 \\
\hline $\mathrm{O}$ & o -11.83253400 & -1.32362200 & -0.77102100 \\
\hline $\mathrm{H}$ & $\begin{array}{ll}-1 & -9.25743000\end{array}$ & $-7 \cdot 30249700$ & -4.27889000 \\
\hline $\mathrm{H}$ & $\begin{array}{ll}-1 & -4.05334800\end{array}$ & 2.90498200 & -0.14437900 \\
\hline $\mathrm{H}$ & $-1 \quad-4.91370900$ & 2.98078300 & -2.38538400 \\
\hline $\mathrm{H}$ & $\begin{array}{ll}-1 & -9.26460000\end{array}$ & 1.09429600 & o -4.27219600 \\
\hline $\mathrm{O}$ & o $\quad-7.55478700$ & -3.10454900 & -6.13853200 \\
\hline $\mathrm{H}$ & $\begin{array}{ll}-1 & -5.85964600\end{array}$ & -4.81024000 & o -6.77135300 \\
\hline $\mathrm{H}$ & $-1 \quad-7.63560500$ & -6.60953000 & o 5.94178500 \\
\hline $\mathrm{H}$ & $-1-11.06386100$ & -0.67560500 & 3.43617000 \\
\hline $\mathrm{H}$ & $\begin{array}{ll}-1 & 0.29823700\end{array}$ & 2.13373200 & -0.61421800 \\
\hline $\mathrm{H}$ & $-1 \quad-1.47116900$ & 2.79429500 & 0.86827900 \\
\hline $\mathrm{H}$ & $-1 \quad 0.22680100$ & 1.27610700 & 1.62541200 \\
\hline $\mathrm{H}$ & $\begin{array}{ll}-1 & -5.59980200\end{array}$ & -10.96117400 & 2.66492800 \\
\hline $\mathrm{H}$ & $-1 \quad-3.36020800$ & -10.88942200 & o $\quad 1.80732400$ \\
\hline $\mathrm{O}$ & o -10.06796ooc & -3.20715300 & -5.19188800 \\
\hline $\mathrm{H}$ & $-1-11.75712500$ & -4.80337300 & -4.27897000 \\
\hline $\mathrm{H}$ & $-1-13.64365600$ & -2.91657700 & 0.07189900 \\
\hline $\mathrm{H}$ & $\begin{array}{ll}-1 & -13.56788100\end{array}$ & -0.67555300 & 0.93221800 \\
\hline $\mathrm{H}$ & $\begin{array}{ll}-1 & -10.13959100\end{array}$ & -9.11346600 & 5.94186300 \\
\hline $\mathrm{H}$ & $\begin{array}{ll}-10.99998700\end{array}$ & -9.18927000 & o $\quad 3.70086300$ \\
\hline $\mathrm{H}$ & $\begin{array}{ll}-1 & -11.75709600\end{array}$ & -7.49127200 & 5.21910300 \\
\hline $\mathrm{H}$ & $\begin{array}{ll}-1 & -9.26456000\end{array}$ & -1.40507600 & -6.77149400 \\
\hline $\mathrm{H}$ & $\begin{array}{ll}-1 & 0.95624000\end{array}$ & 0.40103700 & -2.65003100 \\
\hline $\mathrm{H}$ & $-1 \quad-0.62417800$ & 1.02319600 & -4.34366300 \\
\hline $\mathrm{H}$ & $-1 \quad 0.23351900$ & -1.21645700 & -4.27225700 \\
\hline $\mathrm{H}$ & $-1-11.06388400$ & -3.18123700 & 5.94182900 \\
\hline $\mathrm{H}$ & $-1-11.68608100$ & -4.87489900 & 4.36146700 \\
\hline $\mathrm{H}$ & $\begin{array}{ll}-1 & -7.63562900\end{array}$ & -11.61907400 & 0.93224900 \\
\hline $\mathrm{H}$ & $\begin{array}{ll}-1 & -9.32929300\end{array}$ & -10.03869500 & o $\quad 1.55437100$ \\
\hline $\mathrm{H}$ & $\begin{array}{ll}-1 & -9.25777900\end{array}$ & -10.89634700 & o $\quad-0.68523200$ \\
\hline $\mathrm{H}$ & $-1 \quad-1.54940100$ & -5.53286100 & -6.07822400 \\
\hline $\mathrm{H}$ & $\begin{array}{ll}-1 & -3.24301700\end{array}$ & -3.95253500 & -6.70034000 \\
\hline $\mathrm{H}$ & $\begin{array}{ll}-1 & -1.47366500\end{array}$ & -3.29191000 & -5.21789500 \\
\hline
\end{tabular}




$\begin{array}{lllll}\mathrm{H} & \mathrm{o} & -4.01141700 & -3.85434600 & 0.28882300 \\ \mathrm{O} & \mathrm{o} & -4.75358700 & -3.28089300 & -0.06800800 \\ \mathrm{C} & \mathrm{o} & -2.27966300 & -5.52181000 & -0.97054100 \\ \mathrm{C} & \mathrm{o} & -2.43910500 & -4.62400900 & 1.14686500 \\ \mathrm{C} & \mathrm{o} & -1.47264600 & -4.44142800 & -1.00810700 \\ \mathrm{H} & \mathrm{o} & -2.48370200 & -6.19661700 & -1.79328300 \\ \mathrm{H} & \mathrm{o} & -2.69264700 & -4.46695900 & 2.18838000 \\ \mathrm{C} & \mathrm{o} & -0.71819900 & -3.90949200 & -2.18225700 \\ \mathrm{H} & \mathrm{o} & 0.31152600 & -3.64854300 & -1.91518000 \\ \mathrm{H} & \mathrm{o} & -0.69076700 & -4.64836800 & -2.98735500 \\ \mathrm{H} & \mathrm{o} & -1.19964200 & -3.00124300 & -2.56461300 \\ \mathrm{C} & \mathrm{o} & -1.46804600 & -3.78962000 & 0.35304100 \\ \mathrm{H} & \mathrm{o} & -1.73301100 & -2.72355800 & 0.31916200 \\ \mathrm{H} & \mathrm{o} & -0.46383600 & -3.83658800 & 0.79724100 \\ \mathrm{C} & \mathrm{o} & -2.88389700 & -5.63324000 & 0.36204300 \\ \mathrm{H} & \mathrm{o} & -3.58236400 & -6.40801700 & 0.65912200\end{array}$

\section{$\mathrm{G}^{*}$ (H-FAU)}

(Electronic Energy: -10882.8771373 Ha):

$\begin{array}{lllll}\mathrm{O} & \mathrm{o} & -1.79163900 & 1.22277300 & -0.01819400 \\ \mathrm{O} & \mathrm{o} & -5.31467200 & -8.37168400 & 0.85705100 \\ \mathrm{O} & \mathrm{o} & -5.32749100 & -4.94074200 & -2.66078200 \\ \mathrm{O} & \mathrm{o} & -8.08517000 & 0.59487700 & -0.05834300 \\ \mathrm{O} & \mathrm{o} & -5.33062600 & -5.69589600 & 3.59582100 \\ \mathrm{O} & \mathrm{o} & -10.34447100 & 0.35172100 & -2.49722400 \\ \mathrm{O} & \mathrm{o} & -7.82739300 & -4.85161000 & -5.08866100 \\ \mathrm{O} & \mathrm{o} & -7.01827400 & -3.76990600 & -1.05660200 \\ \mathrm{O} & \mathrm{o} & -9.66044400 & -1.47099500 & -0.69017200 \\ \mathrm{O} & \mathrm{o} & -9.70153000 & -4.06457700 & -3.37138900 \\ \mathrm{O} & \mathrm{o} & -3.39558400 & 2.22067800 & -1.89095800 \\ \mathrm{O} & \mathrm{o} & -12.16092300 & -1.53108900 & 1.71242400 \\ \mathrm{O} & \mathrm{o} & -7.10603500 & -6.74104100 & 1.91325600 \\ \mathrm{O} & \mathrm{o} & -9.72506900 & -6.48152700 & 4.25125900 \\ \mathrm{O} & \mathrm{o} & -8.67750700 & -0.44769600 & -4.41464600 \\ \mathrm{O} & \mathrm{o} & -6.02690000 & -3.00589100 & -4.41244900 \\ \mathrm{O} & \mathrm{o} & -6.29090000 & -0.73748300 & -1.44772600 \\ \mathrm{O} & \mathrm{o} & -7.85607500 & -5.81147900 & -2.57745200 \\ \mathrm{O} & \mathrm{o} & -7.65372400 & -1.80610700 & 1.04009200 \\ \mathrm{O} & \mathrm{o} & -11.35993400 & -2.13705000 & -2.63333900 \\ \mathrm{O} & \mathrm{o} & -1.71624700 & 0.25145900 & -2.51673000 \\ \mathrm{O} & \mathrm{o} & -10.42163300 & -2.16969400 & 3.58671300 \\ \mathrm{O} & \mathrm{o} & -7.84364900 & -4.79080000 & 3.59235300 \\ \mathrm{O} & \mathrm{o} & -7.81937100 & -8.37061800 & -0.05487200 \\ \mathrm{O} & \mathrm{o} & -7.77210300 & 1.21240900 & -2.62094800 \\ \mathrm{O} & \mathrm{o} & -4.41001600 & -2.39191000 & -2.44041400 \\ \mathrm{O} & \mathrm{o} & -3.55624900 & -4.04235300 & -4.31711300 \\ \mathrm{O} & \mathrm{o} & -6.19631800 & -6.33070600 & -0.59338000 \\ \mathrm{O} & \mathrm{o} & -9.75694300 & -0.33687900 & 1.71697500 \\ \mathrm{O} & \mathrm{o} & -6.20484500 & -4.24651800 & 1.61582600 \\ \mathrm{O} & \mathrm{o} & -5.87932900 & 1.85695200 & -0.89606800 \\ \mathrm{O} & \mathrm{o} & -9.68162300 & -4.04077700 & 5.36257700 \\ \mathrm{O} & \mathrm{o} & -3.77071200 & -0.22759700 & -0.97308000\end{array}$




\begin{tabular}{|c|c|c|c|}
\hline $\mathrm{Si}$ & o $\quad-6.63062600$ & -5.20151300 & -1.68569500 \\
\hline $\mathrm{Si}$ & o $\quad-8.76622600$ & -0.79189600 & 0.51486200 \\
\hline $\mathrm{Si}$ & o $\quad-4.83775300$ & -7.02647700 & 4.41668800 \\
\hline $\mathrm{Si}$ & o -10.87152300 & -0.93777000 & -1.64255300 \\
\hline $\mathrm{Al}$ & o $\quad-6.64898200$ & -3.06206300 & 0.47635200 \\
\hline $\mathrm{Si}$ & o $\quad-8.77218100$ & $-5 \cdot 33007900$ & -3.83448500 \\
\hline $\mathrm{Si}$ & o $\quad-4.75429800$ & 2.97489200 & -1.32478300 \\
\hline $\mathrm{Si}$ & o $\quad-6.63382800$ & -5.35504900 & 2.66687700 \\
\hline $\mathrm{Si}$ & o $\quad-9.11716900$ & 0.81363900 & -3.47466700 \\
\hline $\mathrm{Si}$ & o $\quad-6.98355800$ & -3.55978000 & -5.62568200 \\
\hline $\mathrm{Si}$ & o $\quad-4.82255600$ & -1.40462300 & -1.23855500 \\
\hline $\mathrm{Si}$ & o $\quad-2.64875100$ & 0.86921800 & -1.35571100 \\
\hline $\mathrm{Si}$ & o $\quad-6.61609900$ & -7.44342600 & 0.51251700 \\
\hline $\mathrm{Si}$ & o $\quad-8.77579700$ & -5.28717200 & 4.82549400 \\
\hline $\mathrm{Si}$ & o -10.94449400 & -0.94710100 & 2.64544300 \\
\hline $\mathrm{Si}$ & o $\quad-4.85064200$ & -3.60447300 & -3.45395300 \\
\hline $\mathrm{Si}$ & o $\quad-7.00492300$ & 0.74589800 & -1.25878100 \\
\hline $\mathrm{Si}$ & o $\quad-0.91363300$ & 2.54245300 & 0.47418900 \\
\hline $\mathrm{Si}$ & o $\quad-4.84139700$ & -9.22937300 & 2.18295100 \\
\hline $\mathrm{Si}$ & o -10.94998900 & -3.13129800 & -3.86145500 \\
\hline $\mathrm{Si}$ & o -13.09819400 & -0.96628500 & 0.48433800 \\
\hline $\mathrm{Si}$ & o -10.93708900 & -7.45428700 & 4.79637400 \\
\hline $\mathrm{Si}$ & o $\quad-9.19813200$ & -1.37829900 & -5.65133100 \\
\hline $\mathrm{Si}$ & o $\quad-0.49090500$ & 0.78858200 & -3.48322700 \\
\hline $\mathrm{Si}$ & o -10.93421400 & -3.13577100 & 4.81320500 \\
\hline $\mathrm{Si}$ & o $\quad-8.77673100$ & -9.61920000 & 0.45660900 \\
\hline $\mathrm{Si}$ & o $\quad-2.67836200$ & -3.54261100 & -5.62170600 \\
\hline $\mathrm{H}$ & $\begin{array}{ll}-1 & -5.92887300\end{array}$ & -7.48688100 & 5.28066200 \\
\hline $\mathrm{O}$ & o $\quad-4.37993800$ & -8.22544800 & 3.39623300 \\
\hline $\mathrm{H}$ & $\begin{array}{ll}-1 & -3.68929800\end{array}$ & -6.62923500 & 5.20876100 \\
\hline $\mathrm{O}$ & o -12.14543300 & -0.45409800 & -0.75788400 \\
\hline $\mathrm{H}$ & $-1 \quad-9.58663100$ & -6.44725000 & -4.28226300 \\
\hline $\mathrm{H}$ & $-1 \quad-4.38239200$ & $3 \cdot 76037100$ & -0.14770300 \\
\hline $\mathrm{H}$ & $-1 \quad-5.24282000$ & 3.83622700 & -2.38871900 \\
\hline $\mathrm{H}$ & $-1 \quad-9.59362500$ & 1.94960700 & -4.27550700 \\
\hline $\mathrm{O}$ & o $\quad-7.97731800$ & -2.35892100 & -6.10401600 \\
\hline $\mathrm{H}$ & $-1 \quad-6.18869400$ & -3.95485800 & -6.77468000 \\
\hline $\mathrm{H}$ & $\begin{array}{ll}-1 & -7.96465600\end{array}$ & -5.75418900 & 5.93849300 \\
\hline $\mathrm{H}$ & $\begin{array}{ll}-1 & -11.39299200\end{array}$ & 0.17975900 & 3.43290300 \\
\hline $\mathrm{H}$ & $\begin{array}{ll}-1 & -0.03082700\end{array}$ & 2.98906600 & -0.61750300 \\
\hline $\mathrm{H}$ & $\begin{array}{ll}-1 & -1.80021700\end{array}$ & 3.64965300 & 0.86502500 \\
\hline $\mathrm{H}$ & $\begin{array}{ll}-1 & -0.10227400\end{array}$ & 2.13140500 & 1.62210100 \\
\hline $\mathrm{H}$ & $\begin{array}{ll}-1 & -5.92888800\end{array}$ & -10.10590900 & 2.66167700 \\
\hline $\mathrm{H}$ & $-1 \quad-3.68930400$ & -10.03406200 & 1.80402800 \\
\hline $\mathrm{O}$ & o -10.49309900 & -2.25265000 & o $\quad-5.16872300$ \\
\hline $\mathrm{H}$ & $-1-12.08611100$ & -3.94804400 & -4.28228800 \\
\hline $\mathrm{H}$ & $\begin{array}{ll}-1 & -13.97271500\end{array}$ & -2.06124700 & 0.06859200 \\
\hline $\mathrm{H}$ & $\begin{array}{ll}-1 & -13.89692200\end{array}$ & 0.17976500 & 0.92890700 \\
\hline $\mathrm{H}$ & $-1-10.46865600$ & -8.25812400 & 5.93854900 \\
\hline $\mathrm{H}$ & $-1-11.32904800$ & -8.33392300 & 3.69755400 \\
\hline $\mathrm{H}$ & $-1-12.08616000$ & -6.63593300 & 5.21579400 \\
\hline $\mathrm{H}$ & $-1 \quad-9.59360100$ & -0.54981500 & -6.77476600 \\
\hline
\end{tabular}




$\begin{array}{lllll}\mathrm{H} & -1 & 0.62716900 & 1.25637900 & -2.65332900 \\ \mathrm{H} & -1 & -0.95321600 & 1.87855300 & -4.34700400 \\ \mathrm{H} & -1 & -0.09555600 & -0.36112000 & -4.27554800 \\ \mathrm{H} & -1 & -11.39296600 & -2.32589700 & 5.93853700 \\ \mathrm{H} & -1 & -12.01512900 & -4.01955600 & 4.35815800 \\ \mathrm{H} & -1 & -7.96471200 & -10.76376500 & 0.92896600 \\ \mathrm{H} & -1 & -9.65842500 & -9.18337900 & 1.55109900 \\ \mathrm{H} & -1 & -9.58686100 & -10.04102500 & -0.68854700 \\ \mathrm{H} & -1 & -1.87844700 & -4.67752600 & -6.08160500 \\ \mathrm{H} & -1 & -3.57210400 & -3.09714900 & -6.70372300 \\ \mathrm{H} & -1 & -1.80267600 & -2.43652100 & -5.22125200 \\ \mathrm{O} & \mathrm{o} & -4.91776500 & -2.30710400 & 0.19859000 \\ \mathrm{C} & \mathrm{o} & -3.06521800 & -3.93096600 & -0.11747500 \\ \mathrm{C} & \mathrm{o} & -1.96649700 & -3.03483400 & -0.65124100 \\ \mathrm{C} & \mathrm{o} & -1.76205800 & -1.99743200 & 0.16325700 \\ \mathrm{C} & \mathrm{o} & -2.69369600 & -2.05151400 & 1.36050100 \\ \mathrm{C} & \mathrm{o} & -3.77762000 & -3.01696700 & 0.87763000 \\ \mathrm{H} & \mathrm{o} & -3.73672300 & -4.35933900 & -0.86538600 \\ \mathrm{H} & \mathrm{o} & -2.63626100 & -4.77680500 & 0.43710200 \\ \mathrm{H} & \mathrm{o} & -1.40885000 & -3.25232800 & -1.55492700 \\ \mathrm{H} & \mathrm{o} & -1.00486400 & -1.23026500 & 0.04107800 \\ \mathrm{H} & \mathrm{o} & -2.16793300 & -2.62751400 & 2.14072100 \\ \mathrm{H} & \mathrm{o} & -4.26464900 & -3.54224400 & 1.69084500 \\ \mathrm{C} & \mathrm{o} & -3.17689500 & -0.76518800 & 2.02219400 \\ \mathrm{H} & \mathrm{o} & -3.78502900 & -0.15526700 & 1.35573000 \\ \mathrm{H} & \mathrm{o} & -3.77281800 & -1.00726300 & 2.90691400 \\ \mathrm{H} & \mathrm{o} & -2.32121600 & -0.16401400 & 2.33951500 \\ & & & & \end{array}$

$\mathrm{J}^{*}$ (H-FAU)

(Electronic Energy: -10882.8533896 Ha):

$\begin{array}{lllll}\mathrm{O} & \mathrm{o} & -1.79818600 & 1.22992200 & 0.00003300 \\ \mathrm{O} & \mathrm{o} & -5.31779400 & -8.25906900 & 1.03254100 \\ \mathrm{O} & \mathrm{o} & -5.25184700 & -4.64457000 & -2.38683000 \\ \mathrm{O} & \mathrm{o} & -8.01559600 & 0.48171200 & -0.06054500 \\ \mathrm{O} & \mathrm{o} & -5.31173000 & -5.88383800 & 3.46590300 \\ \mathrm{O} & \mathrm{o} & -10.37842000 & 0.35640700 & -2.50759000 \\ \mathrm{O} & \mathrm{o} & -8.04127700 & -4.75819900 & -5.27666400 \\ \mathrm{O} & \mathrm{o} & -7.27216000 & -4.31884900 & -0.63840700 \\ \mathrm{O} & \mathrm{o} & -9.69695300 & -1.48738300 & -0.70888400 \\ \mathrm{O} & \mathrm{o} & -9.71296300 & -4.11095900 & -3.29793500 \\ \mathrm{O} & \mathrm{o} & -3.48841300 & 2.11692400 & -1.86390800 \\ \mathrm{O} & \mathrm{o} & -12.15499200 & -1.51582100 & 1.69289900 \\ \mathrm{O} & \mathrm{o} & -7.07019300 & -6.32861800 & 1.51758800 \\ \mathrm{O} & \mathrm{o} & -9.70994700 & -6.49224900 & 4.24800100 \\ \mathrm{O} & \mathrm{o} & -8.67267000 & -0.42640400 & -4.38804600 \\ \mathrm{O} & \mathrm{o} & -6.13552600 & -3.12431000 & -4.36412400 \\ \mathrm{O} & \mathrm{o} & -6.20269800 & -0.54298500 & -1.73493200 \\ \mathrm{O} & \mathrm{o} & -7.64639500 & -5.76898000 & -2.83327400 \\ \mathrm{O} & \mathrm{o} & -7.73128400 & -1.96212100 & 1.02100700 \\ \mathrm{O} & \mathrm{o} & -11.37359500 & -2.13733800 & -2.66255900 \\ \mathrm{O} & \mathrm{o} & -1.72769300 & 0.25841100 & -2.49779800 \\ \mathrm{O} & \mathrm{o} & -10.44063700 & -2.17477500 & 3.56911900\end{array}$




\begin{tabular}{|c|c|c|c|}
\hline $\mathrm{O}$ & o $\quad-7.80536100$ & -4.83061500 & 3.57453000 \\
\hline $\mathrm{O}$ & o $\quad-7.79076900$ & -8.38104800 & 0.01014500 \\
\hline $\mathrm{O}$ & o $\quad-7.86392400$ & 1.33855300 & -2.57566600 \\
\hline $\mathrm{O}$ & o $\quad-4.32802500$ & -2.13454900 & -2.71768900 \\
\hline $\mathrm{O}$ & o $\quad-3.62160400$ & -4.07898700 & -4.36554300 \\
\hline $\mathrm{O}$ & o $\quad-6.02376900$ & -6.64927400 & o $\quad-0.92683900$ \\
\hline $\mathrm{O}$ & o -9.71833000 & -0.36768400 & 1.72253300 \\
\hline $\mathrm{O}$ & o $\quad-5.95558000$ & -3.89851300 & 1.93848300 \\
\hline $\mathrm{O}$ & o $\quad-5.97681900$ & 1.96324100 & -0.81842900 \\
\hline $\mathrm{O}$ & o $\quad-9.66096300$ & o $\quad-4.03174100$ & $5 \cdot 32493400$ \\
\hline $\mathrm{O}$ & o $\quad-3.69422100$ & -0.34762500 & -0.83849400 \\
\hline $\mathrm{Si}$ & o $\quad-6.57312600$ & -5.30767100 & -1.69619500 \\
\hline $\mathrm{Si}$ & o $\quad-8.76707300$ & -0.86275400 & 0.49296600 \\
\hline $\mathrm{Si}$ & o $\quad-4.80675800$ & -7.11201300 & 4.43171300 \\
\hline $\mathrm{Si}$ & o -10.89659200 & -0.93967000 & -1.66774900 \\
\hline $\mathrm{Al}$ & o $\quad-6.61935000$ & -3.10011900 & 0.37740800 \\
\hline $\mathrm{Si}$ & o $\quad-8.76975600$ & -5.30600700 & -3.91387900 \\
\hline $\mathrm{Si}$ & o -4.78087900 & 2.97449800 & -1.30880300 \\
\hline $\mathrm{Si}$ & o $\quad-6.57447100$ & -5.29365900 & 2.64829900 \\
\hline $\mathrm{Si}$ & o $\quad-9.13937300$ & o.8377780o & -3.46278300 \\
\hline $\mathrm{Si}$ & o $\quad-7.02353000$ & -3.53818400 & -5.67004700 \\
\hline $\mathrm{Si}$ & o $\quad-4.83650000$ & -1.37671300 & -1.36090100 \\
\hline $\mathrm{Si}$ & o $\quad-2.67096600$ & 0.81118900 & -1.30621700 \\
\hline $\mathrm{Si}$ & o $\quad-6.55368500$ & -7.41573100 & 0.39208000 \\
\hline $\mathrm{Si}$ & o $\quad-8.76764500$ & -5.29023600 & 4.81431900 \\
\hline $\mathrm{Si}$ & o -10.94125000 & -0.94300700 & 2.62757200 \\
\hline $\mathrm{Si}$ & o -4.83013100 & -3.49879100 & -3.45664000 \\
\hline $\mathrm{Si}$ & o $\quad-7.00589400$ & 0.80546800 & -1.29479000 \\
\hline $\mathrm{Si}$ & o $\quad-0.91393200$ & 2.54055500 & 0.47545300 \\
\hline $\mathrm{Si}$ & o $\quad-4.82090100$ & -9.24818800 & 2.23876000 \\
\hline $\mathrm{Si}$ & o -10.92472500 & -3.15826400 & -3.85673500 \\
\hline $\mathrm{Si}$ & o -13.10474800 & -0.96107700 & 0.46981200 \\
\hline $\mathrm{Si}$ & o -10.93665700 & -7.45486100 & 4.79566000 \\
\hline $\mathrm{Si}$ & o -9.15629300 & -1.35265700 & -5.64623100 \\
\hline $\mathrm{Si}$ & $0-0.50482400$ & 0.78452100 & -3.47068500 \\
\hline $\mathrm{Si}$ & o -10.93402600 & -3.12973800 & 4.80327200 \\
\hline $\mathrm{Si}$ & o $\quad-8.77635300$ & -9.62392600 & 0.46287500 \\
\hline $\mathrm{Si}$ & o $\quad-2.68607900$ & -3.54655600 & -5.62036600 \\
\hline $\mathrm{H}$ & $\begin{array}{ll}-1 & -5.92889300\end{array}$ & -7.48692600 & 5.28071800 \\
\hline $\mathrm{O}$ & o $\quad-4.30358600$ & -8.37567300 & 3.53264500 \\
\hline $\mathrm{H}$ & $\begin{array}{ll}-1 & -3.68929800\end{array}$ & -6.62924200 & 5.20881600 \\
\hline $\mathrm{O}$ & o -12.16974100 & -0.45390000 & -0.78192700 \\
\hline $\mathrm{H}$ & $-1 \quad-9.58685200$ & -6.44734600 & -4.28222400 \\
\hline $\mathrm{H}$ & $-1 \quad-4.38241400$ & 3.76036700 & -0.14768800 \\
\hline $\mathrm{H}$ & $-1 \quad-5.24279800$ & 3.83614200 & -2.38870300 \\
\hline $\mathrm{H}$ & $\begin{array}{ll}-1 & -9.59359300\end{array}$ & 1.94956200 & -4.27547600 \\
\hline $\mathrm{O}$ & o $\quad-7.88648500$ & o -2.23986600 & o -6.15035300 \\
\hline $\mathrm{H}$ & $-1 \quad-6.18852000$ & -3.95479300 & -6.77478600 \\
\hline $\mathrm{H}$ & $\begin{array}{ll}-1 & -7.96469300\end{array}$ & -5.75420900 & $5 \cdot 93853100$ \\
\hline $\mathrm{H}$ & $\begin{array}{ll}-1 & -11.39295900\end{array}$ & 0.17975200 & 3.43287300 \\
\hline $\mathrm{H}$ & $\begin{array}{ll}-1 & -0.03079100\end{array}$ & 2.98911300 & -0.61753900 \\
\hline $\mathrm{H}$ & $\begin{array}{ll}-1 & -1.80023900\end{array}$ & 3.64970900 & 0.86499200 \\
\hline
\end{tabular}




\begin{tabular}{lllll}
$\mathrm{H}$ & -1 & -0.10225400 & 2.13146100 & 1.62212700 \\
$\mathrm{H}$ & -1 & -5.92888600 & -10.10591500 & 2.66167900 \\
$\mathrm{H}$ & -1 & -3.68928500 & -10.03408300 & 1.80403400 \\
$\mathrm{O}$ & $\mathrm{o}$ & -10.38986300 & -2.31388400 & -5.15683100 \\
$\mathrm{H}$ & -1 & -12.08613900 & -3.94807700 & -4.28223800 \\
$\mathrm{H}$ & -1 & -13.97271000 & -2.06124000 & 0.06859500 \\
$\mathrm{H}$ & -1 & -13.89692400 & 0.17977600 & 0.92890300 \\
$\mathrm{H}$ & -1 & -10.46866100 & -8.25813500 & 5.93855800 \\
$\mathrm{H}$ & -1 & -11.32905600 & -8.33393000 & 3.69755200 \\
$\mathrm{H}$ & -1 & -12.08617500 & -6.63593500 & 5.21580000 \\
$\mathrm{H}$ & -1 & -9.59360700 & -0.54970800 & -6.77479900 \\
$\mathrm{H}$ & -1 & 0.62720900 & 1.25640500 & -2.65333600 \\
$\mathrm{H}$ & -1 & -0.95322600 & 1.87855500 & -4.34700700 \\
$\mathrm{H}$ & -1 & -0.09554800 & -0.36111700 & -4.27554600 \\
$\mathrm{H}$ & -1 & -11.39297200 & -2.32588900 & 5.93853500 \\
$\mathrm{H}$ & -1 & -12.01514600 & -4.01955800 & 4.35816100 \\
$\mathrm{H}$ & -1 & -7.96470300 & -10.76377100 & 0.92896400 \\
$\mathrm{H}$ & -1 & -9.65841200 & -9.18337600 & 1.55109300 \\
$\mathrm{H}$ & -1 & -9.58685300 & -10.04102000 & -0.68855000 \\
$\mathrm{H}$ & -1 & -1.87844300 & -4.67754500 & -6.08164400 \\
$\mathrm{H}$ & -1 & -3.57211800 & -3.09711900 & -6.70377200 \\
$\mathrm{H}$ & -1 & -1.80266700 & -2.43653100 & -5.22125700 \\
$\mathrm{O}$ & $\mathrm{o}$ & -5.13863900 & -2.42826400 & -0.19214000 \\
$\mathrm{C}$ & $\mathrm{o}$ & -3.57868100 & -3.44063900 & 2.26600700 \\
$\mathrm{H}$ & $\mathrm{o}$ & -3.36646300 & -4.44975400 & 2.62827100 \\
$\mathrm{H}$ & $\mathrm{o}$ & -2.85765100 & -2.74936400 & 2.71094800 \\
$\mathrm{C}$ & $\mathrm{o}$ & -4.97003000 & -3.02070600 & 2.68551700 \\
$\mathrm{H}$ & $\mathrm{o}$ & -5.20766200 & -2.02402300 & 2.30561600 \\
$\mathrm{C}$ & $\mathrm{o}$ & -5.16739000 & -3.07834000 & 4.17478000 \\
$\mathrm{H}$ & $\mathrm{o}$ & -4.43696800 & -3.67153700 & 4.71872800 \\
$\mathrm{H}$ & $\mathrm{o}$ & -3.48321200 & -3.39981900 & 1.18164400 \\
$\mathrm{C}$ & $\mathrm{o}$ & -6.11021600 & -2.41379400 & 4.85840900 \\
$\mathrm{C}$ & $\mathrm{o}$ & -7.17401200 & -1.58788000 & 4.29463300 \\
$\mathrm{C}$ & $\mathrm{o}$ & -7.94738100 & -0.82400100 & 5.07185100 \\
$\mathrm{H}$ & $\mathrm{o}$ & -8.72246100 & -0.19269000 & 4.65039800 \\
$\mathrm{H}$ & $\mathrm{o}$ & -7.82007600 & -0.80047900 & 6.15156200 \\
$\mathrm{H}$ & $\mathrm{o}$ & -7.33541800 & -1.60721200 & 3.21840800 \\
$\mathrm{H}$ & $\mathrm{o}$ & -6.09363000 & -2.48576400 & 5.94597400 \\
& & & & \\
\hline
\end{tabular}

$\mathrm{K}^{*}$ (H-FAU)

(Electronic Energy: -10882.8490948 Ha):

$\begin{array}{lllll}\mathrm{O} & \mathrm{o} & -1.82024000 & 1.23419900 & 0.00453500 \\ \mathrm{O} & \mathrm{o} & -5.30666700 & -8.29815300 & 0.98950100 \\ \mathrm{O} & \mathrm{o} & -5.21770100 & -4.64784400 & -2.34900600 \\ \mathrm{O} & \mathrm{o} & -7.72113600 & 0.23545100 & 0.09832100 \\ \mathrm{O} & \mathrm{o} & -5.29752100 & -5.84226800 & 3.48555600 \\ \mathrm{O} & \mathrm{o} & -10.60195100 & 0.29208800 & -2.73184900 \\ \mathrm{O} & \mathrm{o} & -8.07957300 & -4.75657500 & -5.32319000 \\ \mathrm{O} & \mathrm{o} & -7.30277200 & -4.41495500 & -0.64817000 \\ \mathrm{O} & \mathrm{o} & -9.64373600 & -1.31027000 & -0.84666000 \\ \mathrm{O} & \mathrm{o} & -9.69931900 & -4.12623000 & -3.29488400 \\ \mathrm{O} & \mathrm{o} & -3.58985900 & 2.03381300 & -1.83123700\end{array}$




\begin{tabular}{|c|c|c|}
\hline $\mathrm{O}$ & o -12.1697750o & $-1.52913300 \quad 1.70995700$ \\
\hline $\mathrm{O}$ & $0-7.03522400$ & $-6.37605100 \quad 1.51860400$ \\
\hline $\mathrm{O}$ & o -9.71190800 & $-6.48445500 \quad 4.24867400$ \\
\hline $\mathrm{O}$ & o $\quad-8.61170000$ & $-0.37561600 \quad-4.38584500$ \\
\hline $\mathrm{O}$ & o -6.15942500 & $-3.18312000-4.34167100$ \\
\hline $\mathrm{O}$ & o -6.07643400 & $-0.31905200 \quad-1.97129700$ \\
\hline $\mathrm{O}$ & o $\quad-7.58605900$ & $-5.77127200 \quad-2.90944500$ \\
\hline $\mathrm{O}$ & o $\quad-8.05184500$ & $-2.25148700 \quad 1.05854100$ \\
\hline $\mathrm{O}$ & o -11.42685700 & $-2.24052100 \quad-2.57842200$ \\
\hline $\mathrm{O}$ & o -1.74056000 & $0.27180700 \quad-2.49119300$ \\
\hline $\mathrm{O}$ & $0-10.42038500$ & $-2.15026700 \quad 3.57508700$ \\
\hline $\mathrm{O}$ & o -7.78625000 & $-4.84984300 \quad 3.55041100$ \\
\hline $\mathrm{O}$ & o -7.79670300 & $-8.38985800 \quad-0.00539600$ \\
\hline $\mathrm{O}$ & o $\quad-8.13134100$ & $1.28446500 \quad-2.33721500$ \\
\hline $\mathrm{O}$ & o -4.32400600 & $-2.14189000 \quad-2.74287000$ \\
\hline $\mathrm{O}$ & o $\quad-3.62710900$ & $-4.09278500 \quad-4.37295500$ \\
\hline $\mathrm{O}$ & o $\quad-6.01275500$ & $-6.69776200-0.96528800$ \\
\hline $\mathrm{O}$ & o -9.76163800 & $-0.34819400 \quad 1.66462000$ \\
\hline $\mathrm{O}$ & o $\quad-5.92534400$ & $-3.93715100 \quad 1.85959100$ \\
\hline $\mathrm{O}$ & o -6.10099300 & $2.06622500-0.79007400$ \\
\hline $\mathrm{O}$ & o -9.63405900 & $-4.00970100 \quad 5.29009700$ \\
\hline $\mathrm{O}$ & o -3.66608900 & $-0.43514300 \quad-0.80326000$ \\
\hline $\mathrm{Si}$ & o $\quad-6.55566300$ & $-5.34389800 \quad-1.71963100$ \\
\hline $\mathrm{Si}$ & o $\quad-8.78306600$ & $-0.94473400 \quad 0.49942900$ \\
\hline $\mathrm{Si}$ & o -4.80884500 & $-7.09784300 \quad 4.42964200$ \\
\hline $\mathrm{Si}$ & o -10.96027800 & $-0.93577800 \quad-1.72350500$ \\
\hline $\mathrm{Al}$ & o $\quad-6.75383500$ & $-3.14356200 \quad 0.37075300$ \\
\hline $\mathrm{Si}$ & o $\quad-8.75923500$ & $-5.31043100 \quad-3.93738200$ \\
\hline $\mathrm{Si}$ & o $\quad-4.82895600$ & $2.97374600 \quad-1.29198900$ \\
\hline $\mathrm{Si}$ & o $\quad-6.55457500$ & $-5.30488000 \quad 2.61923100$ \\
\hline $\mathrm{Si}$ & o $\quad-9.22757200$ & $0.82016600-3.45140300$ \\
\hline $\mathrm{Si}$ & o $\quad-7.03544300$ & $-3.54485900 \quad-5.67302800$ \\
\hline $\mathrm{Si}$ & o $\quad-4.88423700$ & $-1.31752200 \quad-1.44778900$ \\
\hline $\mathrm{Si}$ & o $\quad-2.69768800$ & $0.77473900 \quad-1.28645700$ \\
\hline $\mathrm{Si}$ & o $\quad-6.54229400$ & $-7.44451400 \quad 0.36420800$ \\
\hline $\mathrm{Si}$ & o $\quad-8.75216700$ & $-5.28878900 \quad 4.79694000$ \\
\hline $\mathrm{Si}$ & o -10.94530700 & $-0.93247500 \quad 2.61904700$ \\
\hline $\mathrm{Si}$ & o $\quad-4.83077500$ & $-3.52053000 \quad-3.45221000$ \\
\hline $\mathrm{Si}$ & o $\quad-7.01423300$ & $0.80566600 \quad-1.25322900$ \\
\hline $\mathrm{Si}$ & o -0.91877800 & $2.53717600 \quad 0.47422700$ \\
\hline $\mathrm{Si}$ & o $\quad-4.82329800$ & $-9.25092100 \quad 2.22782700$ \\
\hline $\mathrm{Si}$ & o -10.92571000 & $-3.17370000-3.82456900$ \\
\hline $\mathrm{Si}$ & o -13.11039000 & $-0.96585100 \quad 0.48098900$ \\
\hline $\mathrm{Si}$ & o -10.93535400 & $-7.45327200 \quad 4.79511300$ \\
\hline $\mathrm{Si}$ & o -9.14157000 & $-1.32164000 \quad-5.61720500$ \\
\hline $\mathrm{Si}$ & o -0.51131000 & $0.78396300 \quad-3.46587400$ \\
\hline $\mathrm{Si}$ & o -10.93148800 & $-3.12241200 \quad 4.79510400$ \\
\hline $\mathrm{Si}$ & o $\quad-8.77920600$ & $-9.62757300 \quad 0.46213600$ \\
\hline $\mathrm{Si}$ & o $\quad-2.68754300$ & $-3.54817100 \quad-5.62008900$ \\
\hline $\mathrm{H}$ & $-1 \quad-5.92884900$ & $-7.48690000 \quad 5.28068300$ \\
\hline $\mathrm{O}$ & o -4.31962500 & $-8.34786900 \quad 3.50511100$ \\
\hline $\mathrm{H}$ & $-1-3.68934600$ & $-6.62925900 \quad 5.20879600$ \\
\hline
\end{tabular}




\begin{tabular}{|c|c|c|}
\hline $\mathrm{O}$ & o -12.17742400 & $-0.45219000 \quad-0.76709700$ \\
\hline $\mathrm{H}$ & $-1 \quad-9.58685200$ & $-6.44734500 \quad-4.28219600$ \\
\hline $\mathrm{H}$ & $-1-4.38252500$ & $3.76038400 \quad-0.14775500$ \\
\hline $\mathrm{H}$ & $-1-5.24272200$ & $3.83612800 \quad-2.38865500$ \\
\hline $\mathrm{H}$ & $-1-9.59351100$ & $1.94921100-4.27503000$ \\
\hline $\mathrm{O}$ & o -7.88507400 & $-2.22784800 \quad-6.12650400$ \\
\hline $\mathrm{H}$ & $-1-6.18851200$ & $-3.95480900 \quad-6.77481200$ \\
\hline $\mathrm{H}$ & $-1 \quad-7.96473100$ & $-5.75416400 \quad 5.93848700$ \\
\hline $\mathrm{H}$ & $-1-11.39287800$ & $0.17975100 \quad 3.43278500$ \\
\hline $\mathrm{H}$ & $-1-0.03082500$ & $2.98909600 \quad-0.61747700$ \\
\hline $\mathrm{H}$ & $-1-1.80021000$ & $3.64972500 \quad 0.86496900$ \\
\hline $\mathrm{H}$ & $-1-0.10226700$ & $2.13146300 \quad 1.62207500$ \\
\hline $\mathrm{H}$ & $-1-5.92884800$ & $-10.10591000 \quad 2.66166400$ \\
\hline $\mathrm{H}$ & $-1-3.68935000$ & $-10.03403100 \quad 1.80405800$ \\
\hline $\mathrm{O}$ & o -10.37621500 & $-2.25023600 \quad-5.06488100$ \\
\hline $\mathrm{H}$ & $-1-12.08609000$ & $-3.94799400-4.28223700$ \\
\hline $\mathrm{H}$ & $-1-13.97258200$ & $-2.06110500 \quad 0.06858600$ \\
\hline $\mathrm{H}$ & $-1-13.89704100$ & $0.17973600 \quad 0.92888200$ \\
\hline $\mathrm{H}$ & $-1-10.46867700$ & $-8.25814300 \quad 5.93855200$ \\
\hline $\mathrm{H}$ & $-1-11.32905100$ & $-8.33392200 \quad 3.69757000$ \\
\hline $\mathrm{H}$ & $-1-12.08616700$ & $-6.63595000 \quad 5.21580300$ \\
\hline $\mathrm{H}$ & $-1 \quad-9.59369500$ & $-0.54965600 \quad-6.77506500$ \\
\hline $\mathrm{H}$ & $-1 \quad 0.62715400$ & $1.25637100 \quad-2.65339600$ \\
\hline $\mathrm{H}$ & $-1 \quad-0.95317300$ & $1.87850900 \quad-4.34699200$ \\
\hline $\mathrm{H}$ & $-1-0.09556600$ & $-0.36102800 \quad-4.27549800$ \\
\hline $\mathrm{H}$ & $-1-11.39301200$ & $-2.32592900 \quad 5.93851300$ \\
\hline $\mathrm{H}$ & $-1-12.01508100$ & $-4.01952100 \quad 4.35818200$ \\
\hline $\mathrm{H}$ & $-1 \quad-7.96471700$ & $-10.76375200 \quad 0.92895100$ \\
\hline $\mathrm{H}$ & $-1 \quad-9.65841500$ & $-9.18338900 \quad 1.55109500$ \\
\hline $\mathrm{H}$ & $-1 \quad-9.58682900$ & $-10.04101400-0.68853200$ \\
\hline $\mathrm{H}$ & $-1-1.87848100$ & $-4.67749700 \quad-6.08159800$ \\
\hline $\mathrm{H}$ & $-1-3.57210600$ & $-3.09712600 \quad-6.70377200$ \\
\hline $\mathrm{H}$ & $-1-1.80267100$ & $-2.43655200 \quad-5.22127100$ \\
\hline $\mathrm{O}$ & o $\quad-5.42655400$ & $-2.30044500-0.31470800$ \\
\hline $\mathrm{C}$ & o -6.65861500 & $-2.23097600 \quad 5.24329600$ \\
\hline $\mathrm{C}$ & o $\quad-6.99729300$ & $-1.80999300 \quad 4.02510500$ \\
\hline $\mathrm{H}$ & o $\quad-5.65849000$ & $-2.07692300 \quad 5.64081200$ \\
\hline $\mathrm{H}$ & o $\quad-7.38428900$ & $-2.72087400 \quad 5.88610300$ \\
\hline $\mathrm{C}$ & o $\quad-6.06357100$ & $-1.11622700 \quad 3.10949900$ \\
\hline $\mathrm{H}$ & o $\quad-8.01203500$ & $-1.95875600 \quad 3.65315600$ \\
\hline $\mathrm{H}$ & o $\quad-6.25311700$ & $-0.05926100 \quad 2.92246100$ \\
\hline $\mathrm{C}$ & o $\quad-5.06478000$ & $-1.70583500 \quad 2.44763400$ \\
\hline $\mathrm{C}$ & o $\quad-4.79223100$ & $-3.18235700 \quad 2.53214000$ \\
\hline $\mathrm{H}$ & o $\quad-4.44865900$ & $-1.13642800 \quad 1.75707300$ \\
\hline $\mathrm{C}$ & o $\quad-3.50752800$ & $-3.61885600 \quad 1.86930000$ \\
\hline $\mathrm{H}$ & o -4.83703000 & $-3.51630300 \quad 3.56812500$ \\
\hline $\mathrm{H}$ & o $\quad-3.38164700$ & $-4.70032300 \quad 1.95248600$ \\
\hline $\mathrm{H}$ & o -3.51080300 & $-3.32501400 \quad 0.81785300$ \\
\hline $\mathrm{H}$ & o -2.66864900 & $-3.12662700 \quad 2.36918100$ \\
\hline
\end{tabular}

$\mathrm{L}^{*}$ (H-FAU)

(Electronic Energy: -10882.8624740 Ha): 


\begin{tabular}{|c|c|c|}
\hline O & -1.81790300 & $1.23049600 \quad 0.02112600$ \\
\hline 0 & $-5 \cdot 32355600$ & $-8.41542300 \quad 0.84201800$ \\
\hline $\mathrm{O}$ & -5.26477000 & $-4.68029600 \quad-2.40111500$ \\
\hline o & -7.96646300 & $0.44599700 \quad-0.04$ \\
\hline o & $-5 \cdot 32127400$ & -5.65958500 \\
\hline o & -10.39279500 & $0.34193100 \quad-2.52573000$ \\
\hline o & -8.03487100 & $-4.75313900 \quad-5.26375000$ \\
\hline o & $-7 \cdot 37601300$ & $-4.12351500-0.82218900$ \\
\hline $\mathrm{O}$ & -9.69812500 & $-1.48473400 \quad-0.72147100$ \\
\hline $\mathrm{O}$ & -9.71163500 & $-4.12024800 \quad-3.29185800$ \\
\hline $\mathrm{O}$ & -3.50455000 & $2.10309500 \quad-1.85316600$ \\
\hline o & -12.14654100 & 1.69737900 \\
\hline o & -7.00124500 & $-6.60857500 \quad 1.79844100$ \\
\hline o & -9.72264600 & $-6.47456500 \quad 4.24752900$ \\
\hline o & -8.65223000 & $-0.41087100 \quad-4.39259700$ \\
\hline o & -6.12088600 & $-3.11510300 \quad-4.36568900$ \\
\hline o & -6.16298000 & $-0.48127900-1.81259100$ \\
\hline $\mathrm{O}$ & -7.64874100 & $-5.81474400 \quad-2.83613500$ \\
\hline $\mathrm{O}$ & $-7 \cdot 74801900$ & $-1.96398700 \quad 1.06256900$ \\
\hline o & -11.38938000 & $-2.15549100 \quad-2.65200900$ \\
\hline o & -1.72265600 & $0.26538000 \quad-2.48254000$ \\
\hline o & -10.40783700 & $-2.14558700 \quad 3.59515200$ \\
\hline o & -7.80801300 & $-4.78610700 \quad 3.57126300$ \\
\hline o & -7.83994400 & $-8.35254300 \quad-0.01939300$ \\
\hline $\mathrm{O}$ & -7.89849400 & $1.36455200 \quad-2.55033200$ \\
\hline $\mathrm{O}$ & $-4 \cdot 31926900$ & $-2.16157300 \quad-2.69597800$ \\
\hline o & -3.61644000 & $-4.08391200 \quad-4.36061800$ \\
\hline o & -6.11853300 & $-6.49355600 \quad-0.76398000$ \\
\hline o & -9.71769600 & $-0.35200200 \quad 1.71260200$ \\
\hline $\mathrm{O}$ & -6.01764900 & $-4.07335200 \quad 1.75061000$ \\
\hline o & -5.99270700 & $1.97850200-0.78830600$ \\
\hline o & -9.64167100 & $-4.01642800 \quad 5.32236000$ \\
\hline o & -3.69497300 & $-0.35925100-0.81194100$ \\
\hline o & -6.63004100 & $-5.24670000 \quad-1.68882000$ \\
\hline $\mathrm{O}$ & -8.77015400 & $-0.88716000 \quad 0.48330800$ \\
\hline o & -4.83756700 & -7.02635800 \\
\hline o & -10.90705600 & $-0.94908800 \quad-1.67570500$ \\
\hline o & -6.64592900 & 0.41305800 \\
\hline O & -8.76445800 & $-5.31696800 \quad-3.90573600$ \\
\hline o & -4.79035600 & $2.97307900 \quad-1.30496600$ \\
\hline o & -6.55012200 & $-5.27882300 \quad 2.64005500$ \\
\hline o & -9.14752500 & $0.83763800 \quad-3.46484900$ \\
\hline o & -7.01656400 & $-3.53747300 \quad-5.66307300$ \\
\hline o & -4.85274200 & $-1.37457200 \quad-1.37104300$ \\
\hline o & -2.68014500 & $0.80066700 \quad-1.29689300$ \\
\hline O & -6.57909200 & $-7.44929100 \quad 0.45058400$ \\
\hline o & -8.75139800 & -5.28896100 \\
\hline o & -10.93477500 & $-0.93933800 \quad 2.62865000$ \\
\hline O & -4.83106800 & $-3.52135400 \quad-3.44629000$ \\
\hline o & -7.00467700 & $0.81338300 \quad-1.30687800$ \\
\hline o & -0.91570900 & $2.53987500 \quad 0.47442900$ \\
\hline o & -4.84370500 & -9.23667700 \\
\hline
\end{tabular}




\begin{tabular}{|c|c|c|c|}
\hline $\mathrm{Si}$ & o -10.92012900 & -3.16819300 & -3.84973700 \\
\hline $\mathrm{Si}$ & o -13.10211800 & -0.96422400 & 0.47464400 \\
\hline $\mathrm{Si}$ & o -10.93441700 & -7.45255600 & 4.79418100 \\
\hline $\mathrm{Si}$ & o -9.14815600 & -1.34879000 & -5.64191000 \\
\hline $\mathrm{Si}$ & $0-0.50511000$ & 0.78399000 & -3.46947800 \\
\hline $\mathrm{Si}$ & o -10.92702700 & -3.13317900 & 4.80615500 \\
\hline $\mathrm{Si}$ & o $\quad-8.77924900$ & -9.61915600 & 0.45991300 \\
\hline $\mathrm{Si}$ & o $\quad-2.68812300$ & -3.54865700 & -5.61738800 \\
\hline $\mathrm{H}$ & $\begin{array}{ll}-1 & -5.92890800\end{array}$ & -7.48698900 & o $\quad 5.28064700$ \\
\hline $\mathrm{O}$ & o $\quad-4.39921200$ & -8.20622800 & 3.37719600 \\
\hline $\mathrm{H}$ & $-1 \quad-3.68932800$ & -6.62925300 & 5.20873400 \\
\hline $\mathrm{O}$ & o -12.16712700 & -0.45391800 & -0.77564600 \\
\hline $\mathrm{H}$ & $\begin{array}{ll}-1 & -9.58682600\end{array}$ & -6.44734100 & -4.28220900 \\
\hline $\mathrm{H}$ & $-1 \quad-4.38242100$ & 3.76035100 & -0.14769000 \\
\hline $\mathrm{H}$ & $\begin{array}{ll}-1 & -5.24278600\end{array}$ & 3.83612400 & -2.38869800 \\
\hline $\mathrm{H}$ & $-1 \quad-9.59359000$ & 1.94955000 & -4.27546700 \\
\hline $\mathrm{O}$ & o -7.88180300 & -2.23852900 & -6.14578200 \\
\hline $\mathrm{H}$ & $-1 \quad-6.18853200$ & -3.95480200 & -6.77476900 \\
\hline $\mathrm{H}$ & $\begin{array}{ll}-1 & -7.96468900\end{array}$ & -5.75422200 & 5.93853800 \\
\hline $\mathrm{H}$ & $\begin{array}{ll}-1 & -11.39295300\end{array}$ & 0.17974900 & 3.43287200 \\
\hline $\mathrm{H}$ & $\begin{array}{ll}-1 & -0.03079800\end{array}$ & 2.98910900 & -0.61753400 \\
\hline $\mathrm{H}$ & $-1-1.80024100$ & 3.64970400 & 0.86499200 \\
\hline $\mathrm{H}$ & $-1 \quad-0.10225300$ & 2.13146300 & 1.62212700 \\
\hline $\mathrm{H}$ & $\begin{array}{ll}-1 & -5.92885700\end{array}$ & -10.10591400 & 2.66171000 \\
\hline $\mathrm{H}$ & $-1 \quad-3.68930600$ & -10.03393800 & 1.80403700 \\
\hline $\mathrm{O}$ & o -10.38148600 & -2.29960700 & -5.13352600 \\
\hline $\mathrm{H}$ & $-1-12.08613000$ & -3.94806900 & -4.28223800 \\
\hline $\mathrm{H}$ & $-1-13.97270600$ & -2.06124300 & 0.06859500 \\
\hline $\mathrm{H}$ & $-1-13.89691800$ & 0.17977500 & 0.92890000 \\
\hline $\mathrm{H}$ & $-1-10.46866800$ & $\begin{array}{ll}0 & -8.25813100\end{array}$ & 5.93855000 \\
\hline $\mathrm{H}$ & $\begin{array}{ll}-1 & -11.32905700\end{array}$ & -8.33392800 & 3.69755800 \\
\hline $\mathrm{H}$ & $-1-12.08616800$ & -6.63594000 & 5.21579600 \\
\hline $\mathrm{H}$ & $\begin{array}{ll}-1 & -9.59360100\end{array}$ & -0.54973100 & -6.77478600 \\
\hline $\mathrm{H}$ & $\begin{array}{ll}-1 & 0.62720700\end{array}$ & 1.25640300 & -2.65334200 \\
\hline $\mathrm{H}$ & $\begin{array}{ll}-1 & -0.95322800\end{array}$ & 1.87854800 & $-4 \cdot 34699800$ \\
\hline $\mathrm{H}$ & $\begin{array}{ll}-1 & -0.09555100\end{array}$ & -0.36111300 & -4.27554400 \\
\hline $\mathrm{H}$ & $\begin{array}{ll}-1 & -11.39296700\end{array}$ & -2.32589700 & 5.93853200 \\
\hline $\mathrm{H}$ & $-1-12.01514200$ & -4.01955600 & $4 \cdot 35816100$ \\
\hline $\mathrm{H}$ & $\begin{array}{ll}-1 & -7.96472000\end{array}$ & -10.76376800 & 0.92898200 \\
\hline $\mathrm{H}$ & $-1 \quad-9.65844800$ & -9.18337400 & 1.55110800 \\
\hline $\mathrm{H}$ & $\begin{array}{ll}-1 & -9.58682500\end{array}$ & -10.04099700 & -0.68853300 \\
\hline $\mathrm{H}$ & $\begin{array}{ll}-1 & -1.87844000\end{array}$ & -4.67754400 & -6.08165200 \\
\hline $\mathrm{H}$ & $-1 \quad-3.57211400$ & -3.09712400 & -6.70375700 \\
\hline $\mathrm{H}$ & $-1 \quad-1.80267100$ & -2.43654200 & -5.22125400 \\
\hline $\mathrm{O}$ & o $\quad-5.20882200$ & -2.35263200 & -0.16860300 \\
\hline $\mathrm{C}$ & o $\quad-6.62463600$ & -0.68749800 & 3.06143400 \\
\hline $\mathrm{C}$ & o $\quad-6.39863400$ & -1.84407900 & 3.78313000 \\
\hline $\mathrm{C}$ & o $\quad-5.10775500$ & -2.26606900 & 3.49552700 \\
\hline $\mathrm{C}$ & o $\quad-5.40954200$ & -0.22183500 & 2.35855100 \\
\hline $\mathrm{H}$ & o $\quad-7.57552000$ & -0.16161100 & 3.02365200 \\
\hline $\mathrm{H}$ & o $\quad-7.14069200$ & -2.38988900 & 4.35095300 \\
\hline $\mathrm{H}$ & o -4.66035100 & -3.18183200 & 3.87777000 \\
\hline
\end{tabular}




$$
\begin{array}{lllll}
\mathrm{H} & \mathrm{o} & -5.07473400 & 0.72719100 & 2.80303100 \\
\mathrm{H} & \mathrm{o} & -5.62049000 & -0.00140400 & 1.30554500 \\
\mathrm{C} & \mathrm{o} & -4.39707800 & -1.35317000 & 2.57831000 \\
\mathrm{H} & \mathrm{o} & -4.31278000 & -1.91693900 & 1.63008900 \\
\mathrm{C} & \mathrm{o} & -2.98757200 & -0.93236300 & 3.00899400 \\
\mathrm{H} & \mathrm{o} & -2.35082600 & -1.80698400 & 3.15824500 \\
\mathrm{H} & \mathrm{o} & -2.54608200 & -0.31941200 & 2.21908000 \\
\mathrm{H} & \mathrm{o} & -3.01089900 & -0.35402200 & 3.93697900
\end{array}
$$

$\mathrm{M}^{*}$ (H-FAU)

(Electronic Energy: -10882.8625757 Ha):
$\mathrm{O}$
$\begin{array}{llll}\text { o } & -1.77531400 & 1.20284900 & -0.00460900\end{array}$
$\mathrm{O}$
$\begin{array}{llll}\text { o } & -5.29610300 & -8.35817100 & 0.87857200\end{array}$
$\mathrm{O}$
$\begin{array}{llll}\text { o } & -5.23558600 & -4.73588500 & -2.40962500\end{array}$
$\mathrm{O}$
$\begin{array}{lllll}\text { o } & -8.02565000 & 0.49296200 & -0.05661300\end{array}$
$\mathrm{O}$
$\begin{array}{llll}\text { o } & -5.29111300 & -5.72513100 & 3.57036800\end{array}$
$\mathrm{O}$
$\begin{array}{llll}\text { o } & -10.38416200 & 0.35844000 & -2.50503800\end{array}$
$\begin{array}{lllll}\text { o } & -8.01500300 & -4.76628000 & -5.24566500\end{array}$
$\begin{array}{lllll}\text { o } & -7.31976300 & -4.00068800 & -0.89465100\end{array}$
$\begin{array}{lllll}\text { o } & -9.67396600 & -1.48591000 & -0.72099200\end{array}$
$\begin{array}{llll}\text { o } & -9.71326600 & -4.11795400 & -3.29392300\end{array}$
$\begin{array}{llll}\text { o } & -3.47740100 & 2.12828200 & -1.85571500\end{array}$
$\begin{array}{llll}\text { o } & -12.17346300 & -1.53284800 & 1.71876700\end{array}$
$\mathrm{O}$
$\begin{array}{llll}\text { o } & -7.08297800 & -6.66727500 & 1.83883300\end{array}$
$\begin{array}{llll}\text { o } & -9.71220900 & -6.48155100 & 4.24437700\end{array}$
$\mathrm{O}$
$\begin{array}{llll}\text { o } & -8.67381200 & -0.42208200 & -4.38845900\end{array}$
$\mathrm{O}$
$\begin{array}{lllll}\text { o } & -6.10060300 & -3.12887300 & -4.36880900\end{array}$
$\begin{array}{llll}\text { o } & -6.19542300 & -0.52949400 & -1.73204100\end{array}$
$\begin{array}{llll}\text { o } & -7.66043100 & -5.81052400 & -2.80674700\end{array}$
$\begin{array}{llll}\text { o } & -7.74494800 & -1.93017100 & 1.07167200\end{array}$
$\begin{array}{llll}\text { o } & -11.36856500 & -2.13365900 & -2.66379100\end{array}$
$\begin{array}{llll}\text { o } & -1.70914600 & 0.23879700 & -2.48340400\end{array}$
$\begin{array}{llll}\text { o } & -10.42386200 & -2.16167800 & 3.58291600\end{array}$
$\begin{array}{llll}\text { o } & -7.79622400 & -4.79783900 & 3.58312200\end{array}$
$\begin{array}{llll}\text { o } & -7.80674500 & -8.38489800 & -0.04600700\end{array}$
$\begin{array}{lllll}\text { o } & -7.86238300 & 1.33957200 & -2.57938300\end{array}$
$\begin{array}{llll}\text { o } & -4.33979200 & -2.20163200 & -2.62610300\end{array}$
$\begin{array}{llll}\text { o } & -3.57652300 & -4.06011300 & -4.31785900\end{array}$
$\begin{array}{llll}\text { o } & -6.14231300 & -6.43070900 & -0.69651100\end{array}$
$\begin{array}{llll}\text { o } & -9.77589600 & -0.33948900 & 1.68330600\end{array}$
$\begin{array}{llll}\text { o } & -6.04120200 & -4.19244300 & 1.65225500\end{array}$
$\begin{array}{lllll}\text { o } & -5.97248100 & 1.95768500 & -0.82114800\end{array}$
$\begin{array}{llll}\text { o } & -9.66464800 & -4.03589200 & 5.33218300\end{array}$
$\begin{array}{llll}0 & -3.66793500 & -0.36839500 & -0.85543100\end{array}$
$\begin{array}{lllll}\text { o } & -6.64157900 & -5.21650400 & -1.67531300\end{array}$
$\begin{array}{lllll}\text { o } & -8.77372600 & -0.85974500 & 0.50894600\end{array}$
$\begin{array}{llll}\text { o } & -4.83112700 & -7.04198700 & 4.41991200\end{array}$
$\begin{array}{llll}\text { o } & -10.88736000 & -0.94418100 & -1.65682500\end{array}$
$\begin{array}{lllll}\text { o } & -6.65162100 & -3.10189600 & 0.44754500\end{array}$
$\begin{array}{lllll}\text { o } & -8.76858300 & -5.31721800 & -3.89304600\end{array}$
$\begin{array}{lllll}\text { o } & -4.79052600 & 2.97670400 & -1.30570700\end{array}$
$\mathrm{Si}$
$\begin{array}{llll}\text { o } & -6.57802300 & -5.31707200 & 2.63744200\end{array}$ 


\begin{tabular}{|c|c|c|c|}
\hline $\mathrm{Si}$ & o $\quad-9.14696300$ & 0.83737200 & -3.45841300 \\
\hline $\mathrm{Si}$ & o $\quad-7.01798900$ & $-3 \cdot 54205700$ & -5.66090700 \\
\hline $\mathrm{Si}$ & o $\quad-4.87298500$ & -1.38248400 & -1.30457000 \\
\hline $\mathrm{Si}$ & o $\quad-2.67673300$ & o.8174970o & -1.31417900 \\
\hline $\mathrm{Si}$ & o $\quad-6.59718400$ & -7.44499400 & 0.48251200 \\
\hline $\mathrm{Si}$ & o $\quad-8.75378100$ & -5.28391000 & 4.80474300 \\
\hline $\mathrm{Si}$ & o -10.9463060o & -0.94299100 & 2.63500200 \\
\hline $\mathrm{Si}$ & o -4.83627000 & -3.53621800 & -3.43125100 \\
\hline $\mathrm{Si}$ & o $\quad-7.02364600$ & 0.80736300 & -1.28682600 \\
\hline $\mathrm{Si}$ & o $\quad-0.91497900$ & 2.53965000 & 0.47404000 \\
\hline $\mathrm{Si}$ & o $\quad-4.83730800$ & -9.23402700 & 2.19357400 \\
\hline $\mathrm{Si}$ & o -10.92288100 & -3.16127000 & -3.85025800 \\
\hline $\mathrm{Si}$ & o - -13.10188300 & -0.96866900 & 0.48535000 \\
\hline $\mathrm{Si}$ & o -10.92989700 & -7.44845300 & 4.79299200 \\
\hline $\mathrm{Si}$ & o $\quad-9.15741900$ & -1.35387300 & -5.64143800 \\
\hline $\mathrm{Si}$ & o $\quad-0.50303700$ & 0.78655000 & -3.47256500 \\
\hline $\mathrm{Si}$ & o -10.93010400 & -3.13244200 & 4.80553700 \\
\hline $\mathrm{Si}$ & о $\quad-8.77750800$ & -9.62211900 & 0.45850500 \\
\hline $\mathrm{Si}$ & o $\quad-2.68861000$ & -3.54710800 & -5.61521600 \\
\hline $\mathrm{H}$ & $\begin{array}{ll}-1 & -5.92889300\end{array}$ & -7.48692600 & o 5.28071800 \\
\hline $\mathrm{O}$ & o $\quad-4.35913000$ & -8.25556100 & 3.42157700 \\
\hline $\mathrm{H}$ & $\begin{array}{ll}-1 & -3.68929800\end{array}$ & -6.62924200 & o $\quad 5.20881600$ \\
\hline $\mathrm{O}$ & o -12.15890500 & -0.46762800 & o -0.76546000 \\
\hline $\mathrm{H}$ & $\begin{array}{ll}-1 & -9.58685200\end{array}$ & -6.44734600 & -4.28222400 \\
\hline $\mathrm{H}$ & $\begin{array}{ll}-1 & -4.38241400\end{array}$ & 3.76036700 & -0.14768800 \\
\hline $\mathrm{H}$ & $-1 \quad-5.24279800$ & 3.83614200 & -2.38870300 \\
\hline $\mathrm{H}$ & $\begin{array}{ll}-1 & -9.59359300\end{array}$ & 1.94956200 & -4.27547600 \\
\hline $\mathrm{O}$ & o $\quad-7.87836800$ & -2.24100600 & -6.13316900 \\
\hline $\mathrm{H}$ & $-1 \quad-6.18852000$ & -3.95479300 & -6.77478600 \\
\hline $\mathrm{H}$ & $\begin{array}{ll}-1 & -7.96469300\end{array}$ & -5.75420900 & 5.93853100 \\
\hline $\mathrm{H}$ & $\begin{array}{ll}-1 & -11.39295900\end{array}$ & 0.17975200 & 3.43287300 \\
\hline $\mathrm{H}$ & $-1 \quad-0.03079100$ & 2.98911300 & -0.61753900 \\
\hline $\mathrm{H}$ & $\begin{array}{ll}-1 & -1.80023900\end{array}$ & 3.64970900 & 0.86499200 \\
\hline $\mathrm{H}$ & $-1 \quad-0.10225400$ & 2.13146100 & 1.62212700 \\
\hline $\mathrm{H}$ & $-1 \quad-5.92888600$ & -10.10591500 & 2.66167900 \\
\hline $\mathrm{H}$ & $\begin{array}{ll}-1 & -3.68928500\end{array}$ & -10.03408300 & o $\quad 1.80403400$ \\
\hline $\mathrm{O}$ & o -10.38441400 & -2.32217600 & -5.15610200 \\
\hline $\mathrm{H}$ & $-1-12.08613900$ & -3.94807700 & -4.28223800 \\
\hline $\mathrm{H}$ & $\begin{array}{ll}-1 & -13.97271000\end{array}$ & -2.06124000 & 0.06859500 \\
\hline $\mathrm{H}$ & $-1-13.89692400$ & 0.17977600 & 0.92890300 \\
\hline $\mathrm{H}$ & $-1-10.46866100$ & -8.25813500 & 5.93855800 \\
\hline $\mathrm{H}$ & $-1-11.32905600$ & -8.33393000 & 3.69755200 \\
\hline $\mathrm{H}$ & $-1-12.08617500$ & -6.63593500 & 5.21580000 \\
\hline $\mathrm{H}$ & $\begin{array}{ll}-1 & -9.59360700\end{array}$ & -0.54970800 & o -6.77479900 \\
\hline $\mathrm{H}$ & $-1 \quad 0.62720900$ & 1.25640500 & -2.65333600 \\
\hline $\mathrm{H}$ & $-1 \quad-0.95322600$ & 1.87855500 & $-4 \cdot 34700700$ \\
\hline $\mathrm{H}$ & $-1 \quad-0.09554800$ & -0.36111700 & -4.27554600 \\
\hline $\mathrm{H}$ & $\begin{array}{ll}-1 & -11.39297200\end{array}$ & -2.32588900 & 5.93853500 \\
\hline $\mathrm{H}$ & $-1-12.01514600$ & -4.01955800 & 4.35816100 \\
\hline $\mathrm{H}$ & $\begin{array}{ll}-1 & -7.96470300\end{array}$ & -10.76377100 & 0.92896400 \\
\hline $\mathrm{H}$ & $-1 \quad-9.65841200$ & -9.18337600 & 1.55109300 \\
\hline $\mathrm{H}$ & $-1 \quad-9.58685300$ & -10.04102000 & -0.68855000 \\
\hline
\end{tabular}




$\begin{array}{lllll}\mathrm{H} & -1 & -1.87844300 & -4.67754500 & -6.08164400 \\ \mathrm{H} & -1 & -3.57211800 & -3.09711900 & -6.70377200 \\ \mathrm{H} & -1 & -1.80266700 & -2.43653100 & -5.22125700 \\ \mathrm{O} & \mathrm{o} & -5.12891400 & -2.37051400 & -0.07736100 \\ \mathrm{C} & \mathrm{o} & -3.21083800 & -3.54913300 & 1.15940200 \\ \mathrm{C} & \mathrm{o} & -2.35055200 & -2.44651200 & 1.17719700 \\ \mathrm{C} & \mathrm{o} & -1.58960000 & -2.20833700 & 0.06258200 \\ \mathrm{C} & \mathrm{o} & -3.12573400 & -4.62345900 & 0.16204200 \\ \mathrm{H} & \mathrm{o} & -4.00244100 & -3.63892900 & 1.90154400 \\ \mathrm{H} & \mathrm{o} & -2.43737700 & -1.69783300 & 1.95526900 \\ \mathrm{H} & \mathrm{o} & -1.06804600 & -1.25417800 & -0.01273700 \\ \mathrm{H} & \mathrm{o} & -4.04469800 & -4.51031000 & -0.43720700 \\ \mathrm{H} & \mathrm{o} & -3.26774300 & -5.58818100 & 0.66628600 \\ \mathrm{C} & \mathrm{o} & -1.87357400 & -4.57399500 & -0.71000100 \\ \mathrm{H} & \mathrm{o} & -1.03102300 & -5.00944800 & -0.16170800 \\ \mathrm{C} & \mathrm{o} & -1.52258000 & -3.13452000 & -1.09195200 \\ \mathrm{H} & \mathrm{o} & -0.53951000 & -3.06166600 & -1.56781700 \\ \mathrm{H} & \mathrm{o} & -2.02808200 & -5.17318900 & -1.60999200 \\ \mathrm{H} & \mathrm{o} & -2.23319900 & -2.72639100 & -1.83432600\end{array}$

\section{$\mathrm{P}^{*}(\mathrm{H}-\mathrm{FAU})$}

(Electronic Energy: -10882.8809284 Ha):

$\begin{array}{lllll}\mathrm{O} & \mathrm{o} & -1.77531400 & 1.20284900 & -0.00460900 \\ \mathrm{O} & \mathrm{o} & -5.29610300 & -8.35817100 & 0.87857200 \\ \mathrm{O} & \mathrm{o} & -5.23558600 & -4.73588500 & -2.40962500 \\ \mathrm{O} & \mathrm{o} & -8.02565000 & 0.49296200 & -0.05661300 \\ \mathrm{O} & \mathrm{o} & -5.2911300 & -5.72513100 & 3.57036800 \\ \mathrm{O} & \mathrm{o} & -10.38416200 & 0.35844000 & -2.50503800 \\ \mathrm{O} & \mathrm{o} & -8.01500300 & -4.76628000 & -5.24566500 \\ \mathrm{O} & \mathrm{o} & -7.31976300 & -4.00068800 & -0.89465100 \\ \mathrm{O} & \mathrm{o} & -9.67396600 & -1.48591000 & -0.72099200 \\ \mathrm{O} & \mathrm{o} & -9.71326600 & -4.11795400 & -3.29392300 \\ \mathrm{O} & \mathrm{o} & -3.47740100 & 2.12828200 & -1.85571500 \\ \mathrm{O} & \mathrm{o} & -12.17346300 & -1.53284800 & 1.71876700 \\ \mathrm{O} & \mathrm{o} & -7.08297800 & -6.66727500 & 1.83883300 \\ \mathrm{O} & \mathrm{o} & -9.71220900 & -6.48155100 & 4.24437700 \\ \mathrm{O} & \mathrm{o} & -8.67381200 & -0.42208200 & -4.38845900 \\ \mathrm{O} & \mathrm{o} & -6.10060300 & -3.12887300 & -4.36880900 \\ \mathrm{O} & \mathrm{o} & -6.19542300 & -0.52949400 & -1.73204100 \\ \mathrm{O} & \mathrm{o} & -7.66043100 & -5.81052400 & -2.80674700 \\ \mathrm{O} & \mathrm{o} & -7.74494800 & -1.93017100 & 1.07167200 \\ \mathrm{O} & \mathrm{o} & -11.36856500 & -2.13365900 & -2.66379100 \\ \mathrm{O} & \mathrm{o} & -1.70914600 & 0.23879700 & -2.48340400 \\ \mathrm{O} & \mathrm{o} & -10.42386200 & -2.16167800 & 3.58291600 \\ \mathrm{O} & \mathrm{o} & -7.79622400 & -4.79783900 & 3.58312200 \\ \mathrm{O} & \mathrm{o} & -7.80674500 & -8.38489800 & -0.04600700 \\ \mathrm{O} & \mathrm{o} & -7.86238300 & 1.33957200 & -2.57938300 \\ \mathrm{O} & \mathrm{o} & -4.33979200 & -2.20163200 & -2.62610300 \\ \mathrm{O} & \mathrm{o} & -3.57652300 & -4.06011300 & -4.31785900 \\ \mathrm{O} & \mathrm{o} & -6.14231300 & -6.43070900 & -0.69651100 \\ \mathrm{O} & \mathrm{o} & -9.77589600 & -0.33948900 & 1.68330600 \\ \mathrm{O} & \mathrm{o} & -6.04120200 & -4.19244300 & 1.65225500 \\ & & & & \\ \mathrm{O} & & & \end{array}$




\begin{tabular}{|c|c|c|c|}
\hline $\mathrm{O}$ & o $\quad-5.97248100$ & 1.95768500 & -0.82114800 \\
\hline $\mathrm{O}$ & o $\quad-9.66464800$ & -4.03589200 & $5 \cdot 33218300$ \\
\hline $\mathrm{O}$ & o $\quad-3.66793500$ & -0.36839500 & -0.85543100 \\
\hline $\mathrm{Si}$ & o $\quad-6.64157900$ & -5.21650400 & -1.67531300 \\
\hline $\mathrm{Si}$ & o $\quad-8.77372600$ & -0.85974500 & 0.50894600 \\
\hline $\mathrm{Si}$ & o $\quad-4.83112700$ & -7.04198700 & 4.41991200 \\
\hline $\mathrm{Si}$ & o -10.88736000 & -0.94418100 & -1.65682500 \\
\hline $\mathrm{Al}$ & o $\quad-6.65162100$ & -3.10189600 & 0.44754500 \\
\hline $\mathrm{Si}$ & o $\quad-8.76858300$ & -5.31721800 & -3.89304600 \\
\hline $\mathrm{Si}$ & o $\quad-4.79052600$ & 2.97670400 & -1.30570700 \\
\hline $\mathrm{Si}$ & o $\quad-6.57802300$ & $-5 \cdot 31707200$ & 2.63744200 \\
\hline $\mathrm{Si}$ & o $\quad-9.14696300$ & 0.83737200 & -3.45841300 \\
\hline $\mathrm{Si}$ & o $\quad-7.01798900$ & -3.54205700 & -5.66090700 \\
\hline $\mathrm{Si}$ & o $\quad-4.87298500$ & -1.38248400 & -1.30457000 \\
\hline $\mathrm{Si}$ & o $\quad-2.67673300$ & 0.81749700 & -1.31417900 \\
\hline $\mathrm{Si}$ & o $\quad-6.59718400$ & -7.44499400 & 0.48251200 \\
\hline $\mathrm{Si}$ & o $\quad-8.75378100$ & -5.28391000 & 4.80474300 \\
\hline $\mathrm{Si}$ & o -10.946306oo & -0.94299100 & 2.63500200 \\
\hline $\mathrm{Si}$ & o $\quad-4.83627000$ & -3.53621800 & -3.43125100 \\
\hline $\mathrm{Si}$ & o $\quad-7.02364600$ & 0.80736300 & -1.28682600 \\
\hline $\mathrm{Si}$ & o $\quad-0.91497900$ & 2.53965000 & 0.47404000 \\
\hline $\mathrm{Si}$ & o -4.83730800 & -9.23402700 & 2.19357400 \\
\hline $\mathrm{Si}$ & o -10.92288100 & -3.16127000 & -3.85025800 \\
\hline $\mathrm{Si}$ & o -13.10188300 & -0.96866900 & 0.48535000 \\
\hline $\mathrm{Si}$ & o -10.92989700 & -7.44845300 & 4.79299200 \\
\hline $\mathrm{Si}$ & o $\quad-9.15741900$ & -1.35387300 & -5.64143800 \\
\hline $\mathrm{Si}$ & o -0.50303700 & 0.78655000 & -3.47256500 \\
\hline $\mathrm{Si}$ & o -10.93010400 & -3.13244200 & 4.80553700 \\
\hline $\mathrm{Si}$ & o $\quad-8.77750800$ & -9.62211900 & 0.45850500 \\
\hline $\mathrm{Si}$ & o $\quad-2.68861000$ & -3.54710800 & -5.61521600 \\
\hline $\mathrm{H}$ & $\begin{array}{ll}-1 & -5.92889300\end{array}$ & -7.48692600 & o 5.28071800 \\
\hline $\mathrm{O}$ & o $\quad-4.35913000$ & -8.25556100 & 3.42157700 \\
\hline $\mathrm{H}$ & $-1 \quad-3.68929800$ & -6.62924200 & o 5.20881600 \\
\hline $\mathrm{O}$ & o -12.15890500 & -0.46762800 & o -0.76546000 \\
\hline $\mathrm{H}$ & $-1 \quad-9.58685200$ & -6.44734600 & $0-4.28222400$ \\
\hline $\mathrm{H}$ & $-1 \quad-4.38241400$ & 3.76036700 & -0.14768800 \\
\hline $\mathrm{H}$ & $\begin{array}{ll}-1 & -5.24279800\end{array}$ & 3.83614200 & -2.38870300 \\
\hline $\mathrm{H}$ & $\begin{array}{ll}-1 & -9.59359300\end{array}$ & 1.94956200 & -4.27547600 \\
\hline $\mathrm{O}$ & o $\quad-7.87836800$ & -2.24100600 & -6.13316900 \\
\hline $\mathrm{H}$ & $-1 \quad-6.18852000$ & -3.95479300 & -6.77478600 \\
\hline $\mathrm{H}$ & $-1 \quad-7.96469300$ & -5.75420900 & 5.93853100 \\
\hline $\mathrm{H}$ & $-1-11.39295900$ & 0.17975200 & 3.43287300 \\
\hline $\mathrm{H}$ & $\begin{array}{ll}-1 & -0.03079100\end{array}$ & 2.98911300 & -0.61753900 \\
\hline $\mathrm{H}$ & $-1 \quad-1.80023900$ & 3.64970900 & 0.86499200 \\
\hline $\mathrm{H}$ & $-1 \quad-0.10225400$ & 2.13146100 & 1.62212700 \\
\hline $\mathrm{H}$ & $\begin{array}{ll}-1 & -5.92888600\end{array}$ & -10.10591500 & 2.66167900 \\
\hline $\mathrm{H}$ & $\begin{array}{ll}-1 & -3.68928500\end{array}$ & -10.03408300 & o 1.80403400 \\
\hline $\mathrm{O}$ & o -10.38441400 & -2.32217600 & -5.15610200 \\
\hline $\mathrm{H}$ & $-1-12.08613900$ & -3.94807700 & -4.28223800 \\
\hline $\mathrm{H}$ & $-1-13.97271000$ & -2.06124000 & 0.06859500 \\
\hline $\mathrm{H}$ & $-1-13.89692400$ & 0.17977600 & 0.92890300 \\
\hline $\mathrm{H}$ & $-1-10.46866100$ & -8.25813500 & 5.93855800 \\
\hline
\end{tabular}




$\begin{array}{lllll}\mathrm{H} & -1 & -11.32905600 & -8.33393000 & 3.69755200 \\ \mathrm{H} & -1 & -12.08617500 & -6.63593500 & 5.21580000 \\ \mathrm{H} & -1 & -9.59360700 & -0.54970800 & -6.77479900 \\ \mathrm{H} & -1 & 0.62720900 & 1.25640500 & -2.65333600 \\ \mathrm{H} & -1 & -0.95322600 & 1.87855500 & -4.34700700 \\ \mathrm{H} & -1 & -0.09554800 & -0.36111700 & -4.27554600 \\ \mathrm{H} & -1 & -11.39297200 & -2.32588900 & 5.93853500 \\ \mathrm{H} & -1 & -12.01514600 & -4.01955800 & 4.35816100 \\ \mathrm{H} & -1 & -7.96470300 & -10.76377100 & 0.92896400 \\ \mathrm{H} & -1 & -9.65841200 & -9.18337600 & 1.55109300 \\ \mathrm{H} & -1 & -9.58685300 & -10.04102000 & -0.68855000 \\ \mathrm{H} & -1 & -1.87844300 & -4.67754500 & -6.08164400 \\ \mathrm{H} & -1 & -3.57211800 & -3.09711900 & -6.70377200 \\ \mathrm{H} & -1 & -1.80266700 & -2.43653100 & -5.22125700 \\ \mathrm{O} & \mathrm{o} & -5.12891400 & -2.37051400 & -0.07736100 \\ \mathrm{C} & \mathrm{o} & -3.21083800 & -3.54913300 & 1.15940200 \\ \mathrm{C} & \mathrm{o} & -2.35055200 & -2.44651200 & 1.17719700 \\ \mathrm{C} & \mathrm{o} & -1.58960000 & -2.20833700 & 0.06258200 \\ \mathrm{C} & \mathrm{o} & -3.12573400 & -4.62345900 & 0.16204200 \\ \mathrm{H} & \mathrm{o} & -4.00244100 & -3.63892900 & 1.90154400 \\ \mathrm{H} & \mathrm{o} & -2.43737700 & -1.69783300 & 1.95526900 \\ \mathrm{H} & \mathrm{o} & -1.06804600 & -1.25417800 & -0.01273700 \\ \mathrm{H} & \mathrm{o} & -4.04469800 & -4.51031000 & -0.43720700 \\ \mathrm{H} & \mathrm{o} & -3.26774300 & -5.58818100 & 0.66628600 \\ \mathrm{C} & \mathrm{o} & -1.87357400 & -4.57399500 & -0.71000100 \\ \mathrm{H} & \mathrm{o} & -1.03102300 & -5.00944800 & -0.16170800 \\ \mathrm{C} & \mathrm{o} & -1.52258000 & -3.13452000 & -1.09195200 \\ \mathrm{H} & \mathrm{o} & -0.53951000 & -3.06166600 & -1.56781700 \\ \mathrm{H} & \mathrm{O} & -2.02808200 & -5.17318900 & -1.60999200 \\ \mathrm{H} & \mathrm{o} & -2.23319900 & -2.72639100 & -1.83432600 \\ & & & & \end{array}$

\section{R-A* TS (H-FAU)}

(Electronic Energy: -10882.8136577 Ha):
$\mathrm{O}$
$\mathrm{O}$
$\begin{array}{llll}\text { o } & -1.43713900 & 0.35547400 & -0.01328000\end{array}$
$\mathrm{O}$
o $\quad-4.97196600 \quad-9.19699200 \quad 0.89463400$
$\mathrm{O}$
$\begin{array}{lllll}\text { o } & -4.88790900 & -5.55040700 & -2.36685100\end{array}$
$\mathrm{O}$
o $\quad-7.76625800 \quad-0.31615200 \quad-0.09246800$
$\mathrm{O}$
o $\quad-4.96982900 \quad-6.60202200 \quad 3.55222400$
o $\quad-10.02957200 \quad-0.47660300 \quad-2.47167900$
$\mathrm{O}$
o $\quad-7.72017200 \quad-5.61631000 \quad-5.27509200$
o $\quad-7.02255800 \quad-4.86922600 \quad-0.88088300$
$\begin{array}{lllll}\text { o } & -9.35864600 & -2.35538100 & -0.70674000\end{array}$
$\mathrm{O}$
o $\quad-9.38278300 \quad-4.97311200 \quad-3.29094100$
$\begin{array}{llll}0 & -3.19296100 & 1.21850700 & -1.83346000\end{array}$
$\mathrm{O}$
$\begin{array}{llll}\text { o } & -11.84131000 & -2.38494400 & 1.71689200\end{array}$
O $\quad \begin{array}{llll}0 & -6.77684600 & -7.50165000 & 1.82266600\end{array}$
O $\quad \begin{array}{lllll}0 & -9.38499900 & -7.33682700 & 4.24897800\end{array}$
$\mathrm{O} \quad \begin{array}{lllll}0 & -8.37262700 & -1.29680200 & -4.38045800\end{array}$
$\mathrm{O} \quad \mathrm{O} \quad-5.79469500 \quad-4.01603200 \quad-4.36299300$
$\mathrm{O} \quad \begin{array}{lllll}0 & -5.84888700 & -1.38949300 & -1.63151500\end{array}$
$\mathrm{O} \quad \begin{array}{lllll}0 & -7.29606700 & -6.63868100 & -2.84011600\end{array}$
O $\quad \begin{array}{lllll}0 & -7.36199000 & -2.71101600 & 1.03297400\end{array}$ 


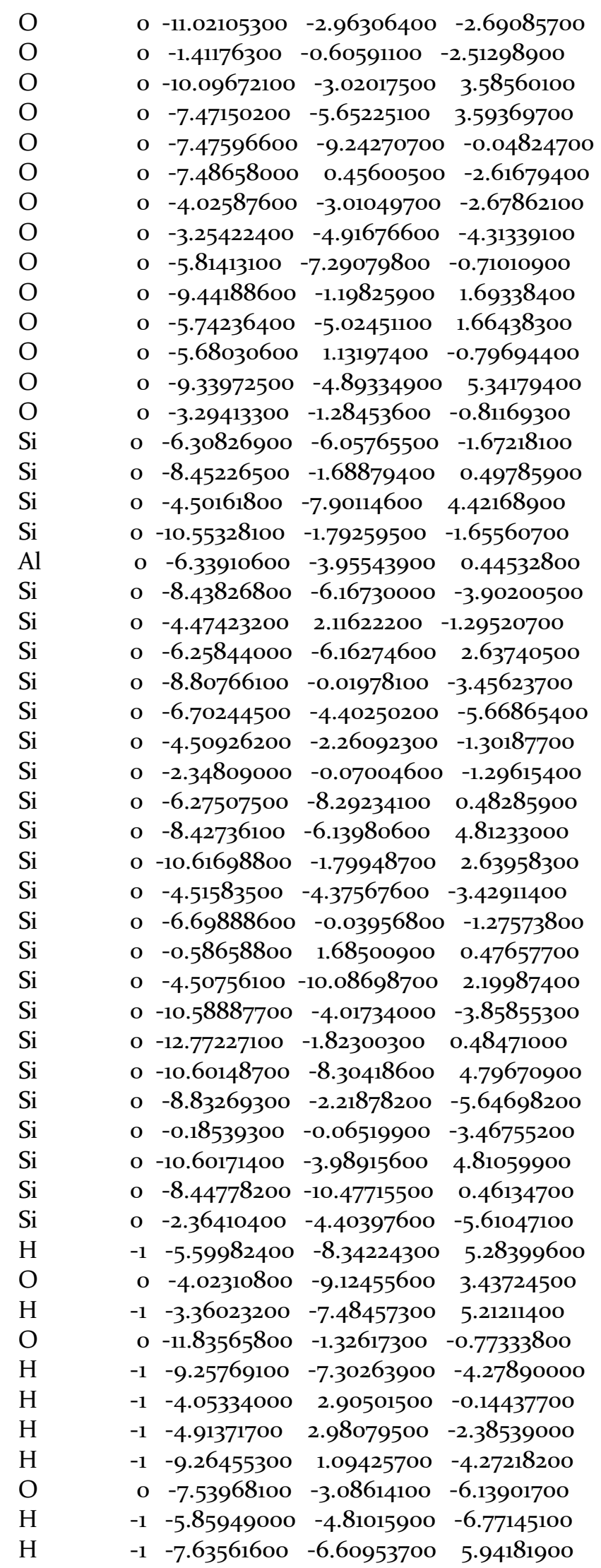




\begin{tabular}{|c|c|c|c|}
\hline $\mathrm{H}$ & $-1-11.06388100$ & -0.67559600 & 3.43618100 \\
\hline $\mathrm{H}$ & $-1 \quad 0.29826200$ & 2.13375200 & -0.61422600 \\
\hline $\mathrm{H}$ & $\begin{array}{ll}-1 & -1.47117400\end{array}$ & 2.79433400 & 0.86828900 \\
\hline $\mathrm{H}$ & $-1 \quad 0.22680800$ & 1.27611200 & 1.62542800 \\
\hline $\mathrm{H}$ & $-1 \quad-5 \cdot 59981300$ & -10.96122200 & 2.66496200 \\
\hline $\mathrm{H}$ & $-1 \quad-3 \cdot 36021300$ & -10.88941500 & 1.80733600 \\
\hline$U$ & o -10.05094000 & o -3.20811900 & -5.18376300 \\
\hline $\mathrm{H}$ & $-1-11.75708200$ & -4.80340800 & -4.27893400 \\
\hline $\mathrm{H}$ & $-1-13.64365000$ & -2.91657900 & 0.07190600 \\
\hline $\mathrm{H}$ & $-1-13.56786700$ & -0.67556100 & 0.93221600 \\
\hline $\mathrm{H}$ & -1 -10.13959000 & -9.11347000 & $5 \cdot 94186600$ \\
\hline $\mathrm{H}$ & $-1 \quad-10.99998700$ & o -9.18926800 & $\begin{array}{l}0 \quad 3.70086200 \\
0\end{array}$ \\
\hline $\mathrm{H}$ & $-1-11.75710200$ & -7.49127300 & 5.21910800 \\
\hline $\mathrm{H}$ & $-1 \quad-9.26454800$ & -1.40504100 & -6.77148900 \\
\hline $\mathrm{H}$ & $\begin{array}{ll}-1 & 0.95626500\end{array}$ & 0.40105300 & -2.65003200 \\
\hline $\mathrm{H}$ & $-1 \quad-0.62417400$ & 1.02320800 & $-4 \cdot 34368000$ \\
\hline $\mathrm{H}$ & $\begin{array}{ll}-1 & 0.23351200\end{array}$ & -1.21645500 & -4.27224300 \\
\hline $\mathrm{H}$ & -1 -11.06389700 & -3.18123000 & $5 \cdot 94184000$ \\
\hline $\mathrm{H}$ & $-1-11.68608000$ & -4.87489800 & $0 \quad 4.36147100$ \\
\hline $\mathrm{H}$ & $\begin{array}{ll}-1 & -7.63562800\end{array}$ & -11.61909600 & 0.93226300 \\
\hline $\mathrm{H}$ & $\begin{array}{ll}-1 & -9.32932400\end{array}$ & -10.03870600 & 1.55439100 \\
\hline $\mathrm{H}$ & $-1 \quad-9.25778000$ & -10.89635200 & -0.68523900 \\
\hline $\mathrm{H}$ & $\begin{array}{ll}-1 & -1.54937800\end{array}$ & $-5 \cdot 53287500$ & -6.07827500 \\
\hline $\mathrm{H}$ & $\begin{array}{ll}-1 & -3.24304200\end{array}$ & -3.95249600 & -6.70041700 \\
\hline $\mathrm{H}$ & $-1 \quad-1.47362300$ & -3.29187200 & -5.21792700 \\
\hline $\mathrm{H}$ & o $\quad-3.83430100$ & -4.66248200 & 0.23979800 \\
\hline $\mathrm{O}$ & o $\quad-4.76934700$ & -3.33782000 & -0.13790900 \\
\hline C & o $\quad-3.14784500$ & -5.63892000 & 0.38745900 \\
\hline C & o $\quad-2.07577800$ & -4.78429300 & 0.09205500 \\
\hline $\mathrm{H}$ & $0 \quad-3 \cdot 31552000$ & $-5 \cdot 92486600$ & 1.42252600 \\
\hline $\mathrm{H}$ & o $\quad-3.50175300$ & -6.32491800 & -0.38179800 \\
\hline C & $0 \quad-1.62678500$ & -4.48690200 & -1.21554500 \\
\hline $\mathrm{H}$ & o -1.65437800 & -4.21699700 & 0.92083300 \\
\hline $\mathrm{H}$ & o $\quad-2.03378500$ & -5.06349800 & -2.04243900 \\
\hline C & $0 \quad-0.68432800$ & $-3 \cdot 52826000$ & -1.45967600 \\
\hline C & $0 \quad-0.05011300$ & -2.65151900 & -0.49740600 \\
\hline $\mathrm{H}$ & $0-0.35212300$ & -3.40551500 & -2.49065900 \\
\hline C & o 1.05352500 & -1.96879800 & -0.83017500 \\
\hline $\mathrm{H}$ & o -0.47657400 & -2.53517500 & 0.49411600 \\
\hline $\mathrm{H}$ & o $\quad 1.55226000$ & -1.31334800 & -0.12412000 \\
\hline 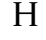 & o 1.49443200 & -2.06056600 & -1.8198290 \\
\hline
\end{tabular}

\section{A-B* TS (H-FAU)}

(Electronic Energy: -10882.7802868 Ha):

$\begin{array}{lllll}\mathrm{O} & \mathrm{o} & -1.80659300 & 1.23087700 & 0.01256400 \\ \mathrm{O} & \mathrm{o} & -5.31860900 & -8.41133900 & 0.84321000 \\ \mathrm{O} & \mathrm{o} & -5.26121400 & -4.73769300 & -2.43543300 \\ \mathrm{O} & \mathrm{o} & -7.95252900 & 0.43292600 & -0.01420500 \\ \mathrm{O} & \mathrm{o} & -5.31095700 & -5.66535800 & 3.63092100 \\ \mathrm{O} & \mathrm{o} & -10.40578900 & 0.34251800 & -2.53209100 \\ \mathrm{O} & \mathrm{o} & -8.00886400 & -4.75774000 & -5.24117200 \\ \mathrm{O} & \mathrm{o} & -7.36450300 & -4.07605800 & -0.90054600\end{array}$




\begin{tabular}{|c|c|c|c|}
\hline $\mathrm{O}$ & o $\quad-9.67267500$ & -1.47395400 & -0.73084100 \\
\hline $\mathrm{O}$ & o $\quad-9.70998400$ & -4.12506100 & -3.29083400 \\
\hline $\mathrm{O}$ & o $\quad-3.50703300$ & 2.10145200 & -1.84739900 \\
\hline $\mathrm{O}$ & o -12.16746000 & -1.53155600 & 1.71610800 \\
\hline $\mathrm{O}$ & o $\quad-7.02905600$ & -6.66294500 & o $\quad 1.83448100$ \\
\hline $\mathrm{O}$ & o $\quad-9.71792800$ & -6.48202200 & 4.24758100 \\
\hline $\mathrm{O}$ & o $\quad-8.65446100$ & -0.40970300 & -4.39236700 \\
\hline $\mathrm{O}$ & o $\quad-6.09968100$ & -3.10377500 & -4.36480100 \\
\hline $\mathrm{O}$ & o $\quad-6.14451300$ & -0.49330600 & -1.77789200 \\
\hline $\mathrm{O}$ & o $\quad-7.66646200$ & -5.85215400 & -2.82191700 \\
\hline $\mathrm{O}$ & o $\quad-7.79259000$ & -2.00789400 & o 1.08823900 \\
\hline $\mathrm{O}$ & o -11.38103500 & -2.15918000 & -2.64417300 \\
\hline $\mathrm{O}$ & o $\quad-1.71451200$ & 0.26772300 & -2.48544500 \\
\hline $\mathrm{O}$ & o - -10.41778900 & -2.15673500 & $3 \cdot 58993300$ \\
\hline $\mathrm{O}$ & o $\quad-7.81372200$ & -4.79508400 & 3.57961900 \\
\hline $\mathrm{O}$ & o $\quad-7.83513700$ & -8.36583500 & -0.03305100 \\
\hline $\mathrm{O}$ & o $\quad-7.89740000$ & 1.33491100 & -2.53198200 \\
\hline $\mathrm{O}$ & o $\quad-4.32793300$ & -2.21097200 & -2.62205000 \\
\hline $\mathrm{O}$ & o $\quad-3.60114100$ & -4.07360500 & $-4 \cdot 34831400$ \\
\hline $\mathrm{O}$ & o $\quad-6.13922300$ & -6.46279600 & -0.72089600 \\
\hline $\mathrm{O}$ & o -9.75702000 & -0.34812300 & 1.69433900 \\
\hline $\mathrm{O}$ & o $\quad-6.07205300$ & -4.13483400 & 1.70145700 \\
\hline $\mathrm{O}$ & o $\quad-5.99634100$ & 1.98087000 & -0.79025900 \\
\hline $\mathrm{O}$ & o $\quad-9.66299800$ & -4.03194800 & D 5.33724200 \\
\hline $\mathrm{O}$ & o $\quad-3.65715400$ & -0.38106900 & -0.81000500 \\
\hline S1 & o $\quad-6.63504200$ & -5.25496300 & -1.70370600 \\
\hline $\mathrm{Si}$ & o $\quad-8.77217700$ & -0.89007000 & 0.51053300 \\
\hline $\mathrm{Si}$ & o $\quad-4.83833800$ & -7.02714700 & 4.41673200 \\
\hline $\mathrm{Si}$ & o - -10.89703900 & -0.95021800 & -1.66721900 \\
\hline $\mathrm{Al}$ & o $\quad-6.68732600$ & -3.14796000 & 0.39004200 \\
\hline $\mathrm{Si}$ & o $\quad-8.76411000$ & $-5 \cdot 32336800$ & -3.89853200 \\
\hline $\mathrm{Si}$ & o $\quad-4.79884800$ & 2.97278400 & -1.30269700 \\
\hline $\mathrm{Si}$ & o $\quad-6.58143900$ & -5.31302800 & 2.64498600 \\
\hline $\mathrm{Si}$ & o $\quad-9.14995200$ & 0.83519200 & -3.45732100 \\
\hline $\mathrm{Si}$ & o $\quad-7.00632700$ & -3.53429200 & -5.65433400 \\
\hline $\mathrm{Si}$ & o $\quad-4.84794600$ & -1.38754700 & -1.31187000 \\
\hline $\mathrm{Si}$ & $\begin{array}{ll}o & -2.68118800\end{array}$ & 0.79883400 & -1.30740600 \\
\hline $\mathrm{Si}$ & o $\quad-6.58940900$ & -7.45887500 & 0.46345200 \\
\hline $\mathrm{Si}$ & o $\quad-8.76156400$ & -5.28727000 & 4.80743000 \\
\hline $\mathrm{Si}$ & o -10.94426800 & -0.94333800 & 2.63423900 \\
\hline $\mathrm{Si}$ & o $\quad-4.82733700$ & -3.53623900 & -3.43374100 \\
\hline $\mathrm{Si}$ & o $\quad-7.00281200$ & 0.79688000 & -1.27977100 \\
\hline $\mathrm{Si}$ & o $\quad-0.91287300$ & 2.54688000 & 0.47213700 \\
\hline $\mathrm{Si}$ & o $\quad-4.84317700$ & -9.23737000 & 2.17762400 \\
\hline $\mathrm{Si}$ & о -10.91921500 & -3.16886700 & -3.84458400 \\
\hline $\mathrm{Si}$ & o -13.10202100 & -0.96816700 & 0.48334400 \\
\hline $\mathrm{Si}$ & o -10.93435100 & -7.45248700 & 4.79437500 \\
\hline $\mathrm{Si}$ & o $\quad-9.14952800$ & -1.34922200 & -5.63876400 \\
\hline $\mathrm{Si}$ & $0 \quad-0.50071100$ & 0.78607100 & -3.47423400 \\
\hline $\mathrm{Si}$ & o -10.93139200 & -3.13273400 & 4.80675700 \\
\hline $\mathrm{Si}$ & o $\quad-8.78148000$ & -9.62286000 & 0.45946600 \\
\hline $\mathrm{Si}$ & $\begin{array}{ll}\mathrm{o} & -2.68724900\end{array}$ & -3.54763300 & -5.61696000 \\
\hline
\end{tabular}




\begin{tabular}{|c|c|c|c|}
\hline $\mathrm{H}$ & -1 & -5.92889300 & $-7.48692600 \quad 5.28071800$ \\
\hline $\mathrm{O}$ & o & -4.39224600 & $-8.21161800 \quad 3.38120300$ \\
\hline $\mathrm{H}$ & -1 & -3.68929800 & $-6.62924200 \quad 5.20881600$ \\
\hline $\mathrm{O}$ & o & -12.15665800 & $-0.46445500 \quad-0.76253700$ \\
\hline $\mathrm{H}$ & -1 & -9.58685200 & $-6.44734600 \quad-4.28222400$ \\
\hline $\mathrm{H}$ & -1 & $-4 \cdot 38241400$ & $3.76036700-0.14768800$ \\
\hline $\mathrm{H}$ & -1 & -5.24279800 & $3.83614200 \quad-2.38870300$ \\
\hline $\mathrm{H}$ & -1 & -9.59359300 & $1.94956200-4.27547600$ \\
\hline 0 & o & -7.88082300 & $-2.24212500 \quad-6.13639600$ \\
\hline $\mathrm{H}$ & -1 & -6.18852000 & $-3.95479300 \quad-6.77478600$ \\
\hline $\mathrm{H}$ & -1 & -7.96469300 & $-5.75420900 \quad 5.93853100$ \\
\hline $\mathrm{H}$ & -1 & -11.39295900 & $0.17975200 \quad 3.43287300$ \\
\hline $\mathrm{H}$ & -1 & -0.03079100 & $2.98911300 \quad-0.61753900$ \\
\hline $\mathrm{H}$ & -1 & -1.80023900 & $3.64970900 \quad 0.86499200$ \\
\hline $\mathrm{H}$ & -1 & -0.10225400 & $2.13146100 \quad 1.62212700$ \\
\hline $\mathrm{H}$ & -1 & $-5 \cdot 92888600$ & $-10.10591500 \quad 2.66167900$ \\
\hline $\mathrm{H}$ & -1 & -3.68928500 & $-10.03408300 \quad 1.80403400$ \\
\hline $\mathrm{O}$ & o & -10.38079100 & $-2.30332100-5.13174200$ \\
\hline $\mathrm{H}$ & -1 & -12.08613900 & $-3.94807700 \quad-4.28223800$ \\
\hline $\mathrm{H}$ & -1 & -13.97271000 & $-2.06124000 \quad 0.06859500$ \\
\hline $\mathrm{H}$ & -1 & -13.89692400 & $0.17977600 \quad 0.92890300$ \\
\hline $\mathrm{H}$ & -1 & -10.46866100 & $-8.25813500 \quad 5.93855800$ \\
\hline $\mathrm{H}$ & -1 & -11.32905600 & -8.33393000 \\
\hline $\mathrm{H}$ & -1 & -12.08617500 & $-6.63593500 \quad 5.21580000$ \\
\hline $\mathrm{H}$ & -1 & -9.59360700 & $-0.54970800 \quad-6.77479900$ \\
\hline $\mathrm{H}$ & -1 & 0.62720900 & $1.25640500 \quad-2.65333600$ \\
\hline $\mathrm{H}$ & -1 & -0.95322600 & $1.87855500 \quad-4 \cdot 34700700$ \\
\hline $\mathrm{H}$ & -1 & -0.09554800 & $-0.36111700-4.27554600$ \\
\hline $\mathrm{H}$ & -1 & -11.392 & $-2.32588900 \quad 5.93853500$ \\
\hline $\mathrm{H}$ & -1 & -12.01514600 & $-4.01955800 \quad 4.35816100$ \\
\hline $\mathrm{H}$ & -1 & $-7 \cdot 96470300$ & $-10.76377100 \quad 0.92896400$ \\
\hline $\mathrm{H}$ & -1 & -9.65841200 & $-9.18337600 \quad 1.55109300$ \\
\hline $\mathrm{H}$ & -1 & -9.58685300 & $-10.04102000-0.68855000$ \\
\hline $\mathrm{H}$ & -1 & -1.87844300 & $-4.67754500-6.08164400$ \\
\hline $\mathrm{H}$ & -1 & $-3 \cdot 57211800$ & $-3.09711900-6.70377200$ \\
\hline $\mathrm{H}$ & -1 & -1.80266700 & $-2.43653100 \quad-5.22125700$ \\
\hline $\mathrm{O}$ & o & -5.20465600 & $-2.33753400-0.08029500$ \\
\hline C & $\mathrm{O}$ & -4.47858100 & $-2.59545500 \quad 2.75261500$ \\
\hline C & $\mathrm{O}$ & -5.47914400 & $-1.60079900 \quad 3.21705800$ \\
\hline $\mathrm{H}$ & o & -4.17930600 & $-3.43939600 \quad 3.37613200$ \\
\hline $\mathrm{H}$ & o & -6.45015400 & $-1.93294000 \quad 2.81750400$ \\
\hline $\mathrm{C}$ & o & -5.13911900 & $-0.25211100 \quad 2.63290700$ \\
\hline $\mathrm{H}$ & o & $-5 \cdot 56849300$ & $4 \cdot 30838900$ \\
\hline $\mathrm{H}$ & o & $-5 \cdot 92186800$ & $0.48939600 \quad 2.51865200$ \\
\hline $\mathrm{C}$ & o & -3.87413000 & $-0.03387100 \quad 2.26193800$ \\
\hline C & $\mathrm{O}$ & -2.90764000 & $-1.10617600 \quad 2.44760000$ \\
\hline $\mathrm{H}$ & o & -3.53115200 & $0.89115100 \quad 1.81203300$ \\
\hline $\mathrm{C}$ & $\mathrm{o}$ & -1.70524200 & $-1.34103000 \quad 1.92874800$ \\
\hline $\mathrm{H}$ & o & $-4 \cdot 32336600$ & $-2.69830200 \quad 1.68349100$ \\
\hline $\mathrm{H}$ & o & -3.22249900 & $-1.85866300 \quad 3.25963400$ \\
\hline $\mathrm{H}$ & o & -1.27257900 & $-0.61064600 \quad 1.25144700$ \\
\hline $\mathrm{H}$ & o & -1.14653200 & -2.23966400 \\
\hline
\end{tabular}


B-C ${ }^{*}$ TS (H-FAU)

(Electronic Energy: -10882.7957910 Ha):

$\mathrm{O}$

$\mathrm{O}$

$\mathrm{O}$

$\mathrm{O}$

$\mathrm{O}$

$\mathrm{O}$

$\mathrm{O}$

$\mathrm{O}$

$\mathrm{O}$

$\mathrm{O}$

$\mathrm{O}$

$\mathrm{O}$

$\mathrm{O}$

$\mathrm{O}$

$\mathrm{O}$

$\mathrm{O}$

$\mathrm{O}$

$\mathrm{O}$

$\mathrm{O}$

$\mathrm{O}$

$\mathrm{O}$

$\mathrm{O}$

$\mathrm{O}$

O

O

O

O

O

O

O

$\mathrm{O}$

$\mathrm{O}$

$\mathrm{O}$

$\mathrm{Si}$

$\mathrm{Si}$

$\mathrm{Si}$

$\mathrm{Si}$

$\mathrm{Al}$

$\mathrm{Si}$

Si

$\mathrm{Si}$

$\mathrm{Si}$

$\mathrm{Si}$

$\mathrm{Si}$

$\mathrm{Si}$

$\mathrm{Si}$

$\mathrm{Si}$

$\mathrm{Si}$

$\mathrm{Si}$ $\begin{array}{llll}\text { o } & -1.83375200 & 1.22688400 & 0.03173300\end{array}$

$\begin{array}{lllll}\text { o } & -5.31708700 & -8.40514400 & 0.85834800\end{array}$

$\begin{array}{llll}\text { o } & -5.24864800 & -4.75026800 & -2.44060100\end{array}$

$\begin{array}{lllll}\text { o } & -7.80539400 & 0.31924400 & 0.07013600\end{array}$

$\begin{array}{lllll}\text { o } & -5.30936700 & -5.68727700 & 3.62401100\end{array}$

$\begin{array}{llll}\text { o } & -10.52554900 & 0.30835100 & -2.65962300\end{array}$

$\begin{array}{llll}\text { o } & -8.00926800 & -4.76010000 & -5.24512300\end{array}$

$\begin{array}{lllll}\text { o } & -7.34613200 & -4.09840900 & -0.90216800\end{array}$

$\begin{array}{lllll}\text { o } & -9.65654600 & -1.36370300 & -0.79500000\end{array}$

$\begin{array}{llll}\text { o } & -9.70509800 & -4.12995400 & -3.29026300\end{array}$

$\begin{array}{llll}\text { o } & -3.54375800 & 2.07594500 & -1.84079300\end{array}$

$\begin{array}{llll}0 & -12.15838200 & -1.52867400 & 1.70580900\end{array}$

$\begin{array}{lllll}\text { o } & -7.02956600 & -6.62489300 & 1.80574200\end{array}$

$\begin{array}{llll}\text { o } & -9.71519400 & -6.47585700 & 4.24549800\end{array}$

$\begin{array}{llll}\text { o } & -8.61275200 & -0.38033500 & -4.39637000\end{array}$

$\begin{array}{llll}\text { o } & -6.09700900 & -3.10653800 & -4.36337300\end{array}$

$\begin{array}{llll}\text { o } & -6.09200900 & -0.39014700 & -1.90463100\end{array}$

$\begin{array}{llll}\text { o } & -7.65349400 & -5.85747600 & -2.83435000\end{array}$

$\begin{array}{lllll}\text { o } & -7.95734300 & -2.15596500 & 1.10438700\end{array}$

$\begin{array}{lllll}0 & -11.41413700 & -2.21509900 & -2.59435100\end{array}$

$\begin{array}{llll}\text { o } & -1.71976700 & 0.27340100 & -2.47661200\end{array}$

$\begin{array}{llll}\text { o } & -10.41316500 & -2.14878400 & 3.58756500\end{array}$

$\begin{array}{llll}\text { o } & -7.79858000 & -4.78876400 & 3.56790600\end{array}$

$\begin{array}{lllll}\text { o } & -7.83077800 & -8.36606200 & -0.02861000\end{array}$

$\begin{array}{lllll}\text { o } & -8.03251400 & 1.31689600 & -2.41704800\end{array}$

$\begin{array}{llll}\text { o } & -4.32598600 & -2.22265200 & -2.61377800\end{array}$

$\begin{array}{llll}\text { o } & -3.59467800 & -4.07095400 & -4.34841100\end{array}$

$\begin{array}{lllll}\text { o } & -6.12117100 & -6.49321400 & -0.74653900\end{array}$

$\begin{array}{llll}\text { o } & -9.75987300 & -0.32722000 & 1.68350400\end{array}$

$\begin{array}{llll}\text { o } & -6.01987900 & -4.11466800 & 1.69947900\end{array}$

$\begin{array}{lllll}\text { o } & -6.03520200 & 2.00944400 & -0.77718800\end{array}$

$\begin{array}{llll}\text { o } & -9.64986700 & -4.02448800 & 5.32241100\end{array}$

$\begin{array}{lllll}\text { o } & -3.66447900 & -0.39456500 & -0.77834100\end{array}$

$\begin{array}{lllll}\text { o } & -6.62143700 & -5.27484100 & -1.70712400\end{array}$

$\begin{array}{lllll}\text { o } & -8.78013000 & -0.93017200 & 0.51948200\end{array}$

$\begin{array}{llll}\text { o } & -4.83461300 & -7.03269300 & 4.42004100\end{array}$

$\begin{array}{llll}\text { o } & -10.93916600 & -0.94272700 & -1.69788200\end{array}$

$\begin{array}{llll}\text { o } & -6.73224500 & -3.17498100 & 0.42772300\end{array}$

$\begin{array}{llll}\text { o } & -8.75925700 & -5.32792100 & -3.89924600\end{array}$

$\begin{array}{llll}\text { o } & -4.81803300 & 2.97477400 & -1.29682400\end{array}$

$\begin{array}{llll}\text { o } & -6.55061100 & -5.28826000 & 2.63200400\end{array}$

$\begin{array}{lllll}\text { o } & -9.19597000 & 0.82507100 & -3.45831600\end{array}$

$\begin{array}{llll}\text { o } & -7.00805000 & -3.53551300 & -5.65310900\end{array}$

$\begin{array}{lllll}\text { o } & -4.88230600 & -1.35091900 & -1.36007600\end{array}$

$\begin{array}{llll}\text { o } & -2.69230200 & 0.79408700 & -1.30133900\end{array}$

$\begin{array}{llll}\text { o } & -6.58400200 & -7.45517300 & 0.46384800\end{array}$

$\begin{array}{lllll}\text { o } & -8.74573500 & -5.28348700 & 4.79436400\end{array}$

$\begin{array}{llll}0 & -10.93999800 & -0.93898600 & 2.63009100\end{array}$

$\begin{array}{llll}\text { o } & -4.82593300 & -3.54834000 & -3.43579200\end{array}$ 


\begin{tabular}{|c|c|c|}
\hline $\mathrm{Si}$ & o $\quad-7.01414300$ & $0.79421900 \quad-1.26310500$ \\
\hline $\mathrm{Si}$ & o -0.91783900 & $2.54229500 \quad 0.47270100$ \\
\hline $\mathrm{Si}$ & o -4.83979700 & $-9.23833100 \quad 2.18690800$ \\
\hline $\mathrm{Si}$ & o -10.92312900 & $-3.17516800 \quad-3.82440500$ \\
\hline $\mathrm{Si}$ & o -13.10554000 & $-0.96680500 \quad 0.48257100$ \\
\hline $\mathrm{Si}$ & o -10.92964700 & $-7.44871000 \quad 4.79258700$ \\
\hline $\mathrm{Si}$ & o $\quad-9.14301400$ & $-1.33316500 \quad-5.62329900$ \\
\hline $\mathrm{Si}$ & $0-0.50245500$ & $0.78530100 \quad-3.47236200$ \\
\hline $\mathrm{Si}$ & o -10.92563300 & $-3.13224100 \quad 4.80337800$ \\
\hline $\mathrm{Si}$ & o $\quad-8.77955000$ & $-9.62136500 \quad 0.45999800$ \\
\hline $\mathrm{Si}$ & o $\quad-2.68509800$ & $-3.54726500 \quad-5.61955700$ \\
\hline $\mathrm{H}$ & $-1 \quad-5.92888500$ & $-7.48689600 \quad 5.28067400$ \\
\hline $\mathrm{O}$ & o -4.38298800 & $\begin{array}{lll}-8.23059100 & 3.39971100\end{array}$ \\
\hline $\mathrm{H}$ & $-1 \quad-3.68930900$ & $-6.62922600 \quad 5.20876600$ \\
\hline $\mathrm{O}$ & o -12.17535400 & $-0.45048800 \quad-0.76919100$ \\
\hline $\mathrm{H}$ & $-1 \quad-9.58683600$ & $-6.44734900-4.28222400$ \\
\hline $\mathrm{H}$ & $-1-4.38241300$ & $3.76037600 \quad-0.14768400$ \\
\hline $\mathrm{H}$ & $-1-5.24280700$ & $3.83614700 \quad-2.38869700$ \\
\hline $\mathrm{H}$ & $-1 \quad-9.59359700$ & $1.94956300-4.27547000$ \\
\hline $\mathrm{O}$ & o $\quad-7.88792700$ & $-2.24333000 \quad-6.12575000$ \\
\hline $\mathrm{H}$ & $-1-6.18850200$ & $-3.95480400-6.77479000$ \\
\hline $\mathrm{H}$ & $-1-7.96466700$ & $-5.75419100 \quad 5.93850000$ \\
\hline $\mathrm{H}$ & $-1-11.39297700$ & $0.17976300 \quad 3.43289600$ \\
\hline $\mathrm{H}$ & $-1-0.03079700$ & $2.98910600-0.61753800$ \\
\hline $\mathrm{H}$ & $-1 \quad-1.80023900$ & $3.64970900 \quad 0.86499100$ \\
\hline $\mathrm{H}$ & $-1-0.10226000$ & $2.13145800 \quad 1.62212400$ \\
\hline $\mathrm{H}$ & $-1 \quad-5.92889300$ & $-10.10592500 \quad 2.66168800$ \\
\hline $\mathrm{H}$ & $-1 \quad-3.68929700$ & $-10.03408200 \quad 1.80403200$ \\
\hline $\mathrm{O}$ & o -10.37814100 & $-2.25900600 \quad-5.07470000$ \\
\hline $\mathrm{H}$ & $-1-12.08611400$ & $-3.94807200-4.28222700$ \\
\hline $\mathrm{H}$ & $-1-13.97270600$ & $-2.06124000 \quad 0.06859700$ \\
\hline $\mathrm{H}$ & $-1-13.89693100$ & $0.17977800 \quad 0.92890400$ \\
\hline $\mathrm{H}$ & $-1-10.46865800$ & $-8.25813200 \quad 5.93855600$ \\
\hline $\mathrm{H}$ & $-1-11.32905300$ & $-8.33392800 \quad 3.69755300$ \\
\hline $\mathrm{H}$ & $-1-12.08616900$ & $-6.63593400 \quad 5.21579800$ \\
\hline $\mathrm{H}$ & $-1 \quad-9.59358700$ & $-0.54971400 \quad-6.77478000$ \\
\hline $\mathrm{H}$ & $-1 \quad 0.62719800$ & $1.25640000-2.65333300$ \\
\hline $\mathrm{H}$ & $-1-0.95322800$ & $1.87855200-4.34700200$ \\
\hline $\mathrm{H}$ & $-1-0.09555500$ & $-0.36111600 \quad-4.27554100$ \\
\hline $\mathrm{H}$ & $-1-11.39296800$ & $-2.32589400 \quad 5.93853700$ \\
\hline $\mathrm{H}$ & $-1-12.01514300$ & $-4.01956000 \quad 4.35816200$ \\
\hline $\mathrm{H}$ & $-1 \quad-7.96471000$ & $-10.76378500 \quad 0.92897100$ \\
\hline $\mathrm{H}$ & $-1-9.65843200$ & $-9.18338400 \quad 1.55110500$ \\
\hline $\mathrm{H}$ & $-1-9.58687200$ & $-10.04103700-0.68855200$ \\
\hline $\mathrm{H}$ & $-1 \quad-1.87843000$ & $-4.67754300-6.08164200$ \\
\hline $\mathrm{H}$ & $-1 \quad-3.57211900$ & $-3.09713600 \quad-6.70375200$ \\
\hline $\mathrm{H}$ & $-1-1.80267100$ & $-2.43652800 \quad-5.22125500$ \\
\hline $\mathrm{O}$ & o $\quad-5.33703500$ & $-2.22632900-0.10262000$ \\
\hline $\mathrm{C}$ & o $\quad-6.22960600$ & $-1.59027000 \quad 3.85805200$ \\
\hline $\mathrm{C}$ & o $\quad-6.32840500$ & $-0.38178600 \quad 2.96811000$ \\
\hline $\mathrm{C}$ & o $\quad-5.30926500$ & $-0.19598300 \quad 2.14523500$ \\
\hline $\mathrm{C}$ & o $\quad-4.27542300$ & $-1.41000600 \quad 2.40851700$ \\
\hline
\end{tabular}




$\begin{array}{lllll}\mathrm{C} & \mathrm{o} & -4.77312300 & -2.08078400 & 3.68436000 \\ \mathrm{H} & \mathrm{o} & -6.47623900 & -1.36645200 & 4.89943800 \\ \mathrm{H} & \mathrm{o} & -6.95062900 & -2.33588700 & 3.50105700 \\ \mathrm{H} & \mathrm{o} & -7.21737000 & 0.24202800 & 2.90856900 \\ \mathrm{H} & \mathrm{o} & -5.16269000 & 0.49949300 & 1.32948900 \\ \mathrm{H} & \mathrm{o} & -4.70563900 & -3.16637100 & 3.60070000 \\ \mathrm{H} & \mathrm{o} & -4.15460000 & -1.75391300 & 4.52769400 \\ \mathrm{C} & \mathrm{o} & -3.02200200 & -0.83204300 & 2.32750200 \\ \mathrm{H} & \mathrm{o} & -2.71248300 & -0.31545200 & 1.41709600 \\ \mathrm{H} & \mathrm{o} & -2.31859000 & -0.84850700 & 3.16264700 \\ \mathrm{H} & \mathrm{o} & -4.51969600 & -1.91017500 & 1.40775500\end{array}$

C-P* TS (H-FAU)

(Electronic Energy: -10882.8410o8o Ha):

$\begin{array}{lllll}\mathrm{O} & \mathrm{o} & -1.46195200 & 0.35540900 & 0.00540500 \\ \mathrm{O} & \mathrm{o} & -4.96220400 & -9.13812400 & 0.97684800 \\ \mathrm{O} & \mathrm{o} & -4.90972100 & -5.55693000 & -2.38526000 \\ \mathrm{O} & \mathrm{o} & -7.54833300 & -0.48096600 & 0.01697300 \\ \mathrm{O} & \mathrm{o} & -4.95172400 & -6.68210100 & 3.50365800 \\ \mathrm{O} & \mathrm{o} & -10.13164900 & -0.53638000 & -2.58332800 \\ \mathrm{O} & \mathrm{o} & -7.69363200 & -5.62003900 & -5.25975300 \\ \mathrm{O} & \mathrm{o} & -6.99653900 & -5.02100300 & -0.77024000 \\ \mathrm{O} & \mathrm{o} & -9.31595200 & -2.29789200 & -0.76000300 \\ \mathrm{O} & \mathrm{o} & -9.37790200 & -4.97709300 & -3.29066700 \\ \mathrm{O} & \mathrm{o} & -3.13395000 & 1.29243100 & -1.85804000 \\ \mathrm{O} & \mathrm{o} & -11.85313200 & -2.39296300 & 1.73263800 \\ \mathrm{O} & \mathrm{o} & -6.79634500 & -7.37808100 & 1.70514800 \\ \mathrm{O} & \mathrm{o} & -9.36897400 & -7.33634500 & 4.24047100 \\ \mathrm{O} & \mathrm{o} & -8.30239400 & -1.24779900 & -4.38711000 \\ \mathrm{O} & \mathrm{o} & -5.78106300 & -3.99007300 & -4.35562300 \\ \mathrm{O} & \mathrm{o} & -5.84912400 & -1.33881600 & -1.88611700 \\ \mathrm{O} & \mathrm{o} & -7.31466800 & -6.66508200 & -2.83227300 \\ \mathrm{O} & \mathrm{o} & -7.54050200 & -2.93440500 & 1.12997000 \\ \mathrm{O} & \mathrm{o} & -11.06877200 & -3.04434100 & -2.60940100 \\ \mathrm{O} & \mathrm{o} & -1.39639100 & -0.60434800 & -2.48808900 \\ \mathrm{O} & \mathrm{o} & -10.09131300 & -3.01133800 & 3.58345500 \\ \mathrm{O} & \mathrm{o} & -7.41841300 & -5.64570800 & 3.59611200 \\ \mathrm{O} & \mathrm{o} & -7.45505100 & -9.26288500 & -0.02749600 \\ \mathrm{O} & \mathrm{o} & -7.63964700 & 0.49518500 & -2.47611800 \\ \mathrm{O} & \mathrm{o} & -3.97996000 & -3.05496700 & -2.63843600 \\ \mathrm{O} & \mathrm{o} & -3.26566500 & -4.93865000 & -4.33579400 \\ \mathrm{O} & \mathrm{o} & -5.75118700 & -7.39309800 & -0.79459500 \\ \mathrm{O} & \mathrm{o} & -9.45591900 & -1.19580300 & 1.66632600 \\ \mathrm{O} & \mathrm{o} & -5.56715000 & -4.96685600 & 1.72429300 \\ \mathrm{O} & \mathrm{o} & -5.64078500 & 1.10108700 & -0.83204900 \\ \mathrm{O} & \mathrm{o} & -9.32434500 & -4.88522700 & 5.31350200 \\ \mathrm{O} & \mathrm{o} & -3.40023600 & -1.16766800 & -0.84734000 \\ \mathrm{Si} & \mathrm{o} & -6.29163500 & -6.12309700 & -1.68436300 \\ \mathrm{Si} & \mathrm{o} & -8.43721500 & -1.76365200 & 0.52572600 \\ \mathrm{Si} & \mathrm{o} & -4.48814000 & -7.94654600 & 4.43361000 \\ \mathrm{Si} & \mathrm{o} & -10.57467700 & -1.80970000 & -1.664487300 \\ \mathrm{Al} & \mathrm{o} & -6.34897400 & -3.98118300 & 0.46583900 \\ & & & & \\ \mathrm{O} & & & \\ \mathrm{O} & & \end{array}$




\begin{tabular}{|c|c|c|}
\hline $\mathrm{Si}$ & o $\quad-8.43678200$ & $-6.17094500 \quad-3.90455900$ \\
\hline Si & o -4.45243200 & $2.12171800 \quad-1.30655300$ \\
\hline Si & o -6.22426000 & $-6.14149400 \quad 2.61546100$ \\
\hline $\mathrm{Si}$ & o $\quad-8.84441200$ & $-0.01968800 \quad-3.44926100$ \\
\hline $\mathrm{Si}$ & o -6.68827800 & $-4.39368100 \quad-5.65744200$ \\
\hline $\mathrm{Si}$ & o $\quad-4.55434200$ & $-2.19691000 \quad-1.36593400$ \\
\hline $\mathrm{Si}$ & $0 \quad-2.35051700$ & $-0.03317400 \quad-1.30872900$ \\
\hline $\mathrm{Si}$ & o -6.26094700 & $-8.28757200 \quad 0.45348000$ \\
\hline $\mathrm{Si}$ & o $\quad-8.41261700$ & $-6.13448500 \quad 4.79940000$ \\
\hline $\mathrm{Si}$ & o -10.61830300 & $-1.79512700 \quad 2.63282500$ \\
\hline $\mathrm{Si}$ & o -4.49626100 & $-4.38051900 \quad-3.43331900$ \\
\hline $\mathrm{Si}$ & o $\quad-6.67701100$ & $-0.06480900 \quad-1.28601200$ \\
\hline $\mathrm{Si}$ & o -0.58773000 & $1.68166200 \quad 0.47698700$ \\
\hline $\mathrm{Si}$ & o -4.49744400 & $-10.09716100 \quad 2.22650300$ \\
\hline $\mathrm{Si}$ & o -10.59684800 & $-4.01995400 \quad-3.82970300$ \\
\hline $\mathrm{Si}$ & o -12.77483200 & $-1.82593300 \quad 0.49437400$ \\
\hline $\mathrm{Si}$ & o -10.59532100 & $-8.29963600 \quad 4.79352400$ \\
\hline $\mathrm{Si}$ & o $\quad-8.81831000$ & $-2.18922000 \quad-5.62265900$ \\
\hline $\mathrm{Si}$ & o -0.17786400 & $-0.07037700-3.46605000$ \\
\hline $\mathrm{Si}$ & o -10.60039000 & $-3.98259700 \quad 4.80258800$ \\
\hline $\mathrm{Si}$ & o -8.45003800 & $-10.48410900 \quad 0.46410600$ \\
\hline $\mathrm{Si}$ & o $\quad-2.35862000$ & $-4.40309100-5.61299000$ \\
\hline $\mathrm{H}$ & $-1 \quad-5.59979100$ & $-8.34237400 \quad 5.28419900$ \\
\hline $\mathrm{O}$ & o $\quad-3.98489300$ & $-9.19137400 \quad 3.49872300$ \\
\hline $\mathrm{H}$ & $-1-3.36056000$ & $-7.48483700 \quad 5.21131400$ \\
\hline $\mathrm{O}$ & o -11.82079800 & $-1.32358800 \quad-0.74531500$ \\
\hline $\mathrm{H}$ & $-1 \quad-9.25755100$ & $-7.30255700 \quad-4.27873800$ \\
\hline $\mathrm{H}$ & $-1-4.05337800$ & $2.90499500 \quad-0.14440900$ \\
\hline $\mathrm{H}$ & $-1 \quad-4.91369100$ & $2.98074300 \quad-2.38535800$ \\
\hline $\mathrm{H}$ & $-1 \quad-9.26450900$ & $1.09417600 \quad-4.27209100$ \\
\hline $\mathrm{O}$ & o -7.55561800 & $-3.09481700 \quad-6.12293800$ \\
\hline $\mathrm{H}$ & $-1-5.85946200$ & $-4.81017800-6.77140900$ \\
\hline $\mathrm{H}$ & $-1-7.63562600$ & $-6.60957000 \quad 5.94172700$ \\
\hline $\mathrm{H}$ & $-1-11.06381500$ & $-0.67566300 \quad 3.43612000$ \\
\hline $\mathrm{H}$ & $-1 \quad 0.29823900$ & $2.13375900 \quad-0.61420400$ \\
\hline $\mathrm{H}$ & $-1 \quad-1.47115700$ & $2.79432400 \quad 0.86828400$ \\
\hline $\mathrm{H}$ & $-1 \quad 0.22679900$ & $1.27612100 \quad 1.62541100$ \\
\hline $\mathrm{H}$ & $-1 \quad-5.59978200$ & $-10.96139000 \quad 2.66506000$ \\
\hline $\mathrm{H}$ & $-1 \quad-3.36049400$ & $-10.88869100 \quad 1.80761700$ \\
\hline $\mathrm{O}$ & o -10.05110300 & $-3.13381900 \quad-5.09984400$ \\
\hline $\mathrm{H}$ & $-1-11.75708500$ & $-4.80344100-4.27892000$ \\
\hline $\mathrm{H}$ & $-1-13.64366200$ & $-2.91658200 \quad 0.07191800$ \\
\hline $\mathrm{H}$ & $-1-13.56782200$ & $-0.67557600 \quad 0.93220100$ \\
\hline $\mathrm{H}$ & $-1-10.13958300$ & $-9.11347400 \quad 5.94192000$ \\
\hline $\mathrm{H}$ & $-1-10.99994400$ & $\begin{array}{lll}0 & -9.18928500 & 3.70083700\end{array}$ \\
\hline $\mathrm{H}$ & $-1-11.75709000$ & $-7.49123200 \quad 5.21909600$ \\
\hline $\mathrm{H}$ & $-1 \quad-9.26456300$ & $-1.40500500-6.77147700$ \\
\hline $\mathrm{H}$ & $-1 \quad 0.95626100$ & $0.40106000 \quad-2.65003700$ \\
\hline $\mathrm{H}$ & $-1-0.62416600$ & $1.02321300-4.34368100$ \\
\hline $\mathrm{H}$ & $-1 \quad 0.23351500$ & $-1.21645700 \quad-4.27225600$ \\
\hline $\mathrm{H}$ & $-1-11.06381900$ & $-3.18125300 \quad 5.94180600$ \\
\hline $\mathrm{H}$ & $-1-11.68608600$ & $-4.87491800 \quad 4.36148300$ \\
\hline
\end{tabular}




$\begin{array}{lllll}\mathrm{H} & -1 & -7.63561500 & -11.61911800 & 0.93224400 \\ \mathrm{H} & -1 & -9.32938700 & -10.03871300 & 1.55448400 \\ \mathrm{H} & -1 & -9.25760900 & -10.89625500 & -0.68520000 \\ \mathrm{H} & -1 & -1.54936300 & -5.53285800 & -6.07831900 \\ \mathrm{H} & -1 & -3.24300200 & -3.95251700 & -6.70037300 \\ \mathrm{H} & -1 & -1.47365300 & -3.29188400 & -5.21796000 \\ \mathrm{H} & \mathrm{o} & -3.69522300 & -3.14484500 & 1.58461600 \\ \mathrm{O} & \mathrm{o} & -4.93930000 & -3.14478200 & -0.13918500 \\ \mathrm{C} & \mathrm{o} & -3.22809600 & -5.10996600 & 1.54373600 \\ \mathrm{C} & \mathrm{o} & -1.37733900 & -4.07983800 & -0.34107300 \\ \mathrm{C} & \mathrm{o} & -1.78938400 & -3.28622300 & 0.64401800 \\ \mathrm{C} & \mathrm{o} & -2.76466500 & -3.73617500 & 1.70593900 \\ \mathrm{H} & \mathrm{o} & -3.47097700 & -5.68538200 & 2.43109400 \\ \mathrm{H} & \mathrm{o} & -0.66686200 & -3.69869300 & -1.06948300 \\ \mathrm{H} & \mathrm{o} & -1.44388900 & -2.26008700 & 0.72481000 \\ \mathrm{H} & \mathrm{o} & -2.41561700 & -3.56109300 & 2.72831800 \\ \mathrm{C} & \mathrm{o} & -1.84614900 & -5.49760800 & -0.50646000 \\ \mathrm{H} & \mathrm{o} & -2.03433900 & -5.72116800 & -1.56105400 \\ \mathrm{H} & \mathrm{o} & -1.07459000 & -6.20078100 & -0.16991600 \\ \mathrm{C} & \mathrm{o} & -3.18307700 & -5.74997700 & 0.23553900 \\ \mathrm{H} & \mathrm{o} & -3.47553700 & -6.80034200 & 0.26244600 \\ \mathrm{H} & \mathrm{o} & -3.92395900 & -5.19374400 & -0.36054700\end{array}$

F-B* TS (H-FAU)

(Electronic Energy: -10882.8805677 Ha):

$\begin{array}{lllll}\mathrm{O} & \mathrm{o} & -1.46626500 & 0.37472900 & 0.00476700 \\ \mathrm{O} & \mathrm{o} & -4.98044600 & -9.20354600 & 0.89384100 \\ \mathrm{O} & \mathrm{o} & -4.93414700 & -5.52535300 & -2.38763800 \\ \mathrm{O} & \mathrm{o} & -7.79999300 & -0.24358100 & -0.09548000 \\ \mathrm{O} & \mathrm{o} & -4.96933000 & -6.58198800 & 3.57479300 \\ \mathrm{O} & \mathrm{o} & -10.01211500 & -0.48499200 & -2.47401800 \\ \mathrm{O} & \mathrm{o} & -7.69377900 & -5.62240500 & -5.25365500 \\ \mathrm{O} & \mathrm{o} & -7.02586200 & -4.87446300 & -0.86938600 \\ \mathrm{O} & \mathrm{o} & -9.34183000 & -2.33697700 & -0.69217500 \\ \mathrm{O} & \mathrm{o} & -9.38151900 & -4.96868600 & -3.29516300 \\ \mathrm{O} & \mathrm{o} & -3.13651200 & 1.27970900 & -1.86999700 \\ \mathrm{O} & \mathrm{o} & -11.82770000 & -2.38037300 & 1.70870800 \\ \mathrm{O} & \mathrm{o} & -6.75782800 & -7.46828200 & 1.81239100 \\ \mathrm{O} & \mathrm{o} & -9.38474300 & -7.33534900 & 4.24672800 \\ \mathrm{O} & \mathrm{o} & -8.35990800 & -1.29872800 & -4.39191900 \\ \mathrm{O} & \mathrm{o} & -5.79624400 & -3.96466100 & -4.37422100 \\ \mathrm{O} & \mathrm{o} & -5.94613900 & -1.47466900 & -1.55554000 \\ \mathrm{O} & \mathrm{o} & -7.32880700 & -6.65275600 & -2.81229900 \\ \mathrm{O} & \mathrm{o} & -7.27781200 & -2.61285000 & 0.98266000 \\ \mathrm{O} & \mathrm{o} & -11.01258100 & -2.97269400 & -2.66983100 \\ \mathrm{O} & \mathrm{o} & -1.39640600 & -0.61185900 & -2.48894600 \\ \mathrm{O} & \mathrm{o} & -10.09560000 & -3.02509600 & 3.58636800 \\ \mathrm{O} & \mathrm{o} & -7.47461800 & -5.64245300 & 3.58793900 \\ \mathrm{O} & \mathrm{o} & -7.48652300 & -9.22890800 & -0.03460800 \\ \mathrm{O} & \mathrm{o} & -7.46517900 & 0.44077800 & -2.63586600 \\ \mathrm{O} & \mathrm{o} & -4.03079600 & -3.01249300 & -2.67648500 \\ \mathrm{O} & \mathrm{o} & -3.27533600 & -4.91780700 & -4.33601600\end{array}$




\begin{tabular}{|c|c|c|c|}
\hline $\mathrm{O}$ & o $\quad-5.81616400$ & -7.29049700 & -0.71052200 \\
\hline $\mathrm{O}$ & o $\quad-9.41575400$ & -1.20525800 & 1.71883500 \\
\hline $\mathrm{O}$ & o $\quad-5.73384100$ & -4.97892900 & 1.69258800 \\
\hline $\mathrm{O}$ & o $\quad-5.61113900$ & 1.06824700 & -0.84205300 \\
\hline $\mathrm{O}$ & o $\quad-9.33782900$ & -4.89377700 & o $\quad 5 \cdot 34191400$ \\
\hline $\mathrm{O}$ & o $\quad-3.39072400$ & -1.17158300 & -0.84221000 \\
\hline $\mathrm{Si}$ & o $\quad-6.31734300$ & -6.07099400 & -1.67163400 \\
\hline $\mathrm{Si}$ & o $\quad-8.44170900$ & -1.63857100 & 0.49605100 \\
\hline $\mathrm{Si}$ & o $\quad-4.50021000$ & -7.89697100 & 4.42425900 \\
\hline $\mathrm{Si}$ & o -10.54413100 & -1.79196000 & -1.64934000 \\
\hline $\mathrm{Al}$ & o $\quad-6.40280100$ & -3.98039300 & 0.47191400 \\
\hline $\mathrm{Si}$ & o $\quad-8.44144900$ & -6.17028800 & -3.89782000 \\
\hline $\mathrm{Si}$ & o $\quad-4.44346200$ & 2.12085500 & -1.31048000 \\
\hline $\mathrm{Si}$ & o $\quad-6.24196100$ & -6.14788500 & 2.65041500 \\
\hline $\mathrm{Si}$ & o $\quad-8.79927500$ & -0.02530100 & -3.46833400 \\
\hline $\mathrm{Si}$ & o $\quad-6.69945400$ & -4.39754500 & -5.67079900 \\
\hline $\mathrm{Si}$ & o -4.51271000 & -2.19864000 & -1.36591100 \\
\hline $\mathrm{Si}$ & $\begin{array}{ll}0 & -2.33235000\end{array}$ & -0.02685200 & -1.30613100 \\
\hline $\mathrm{Si}$ & o $\quad-6.27201600$ & -8.29026100 & 0.48423200 \\
\hline $\mathrm{Si}$ & o $\quad-8.42553800$ & -6.14025100 & 4.81059700 \\
\hline $\mathrm{Si}$ & o -10.61275500 & -1.80129100 & 2.64550800 \\
\hline $\mathrm{Si}$ & o $\quad-4.52168500$ & -4.37783500 & -3.45465100 \\
\hline $\mathrm{Si}$ & o $\quad-6.71070300$ & -0.03811900 & -1.27456000 \\
\hline $\mathrm{Si}$ & o $\quad-0.58128500$ & 1.69094300 & 0.48195000 \\
\hline $\mathrm{Si}$ & o $\quad-4.50695000$ & -10.08867400 & o $\quad 2.19920600$ \\
\hline $\mathrm{Si}$ & o -10.59244700 & -4.01564200 & -3.85392300 \\
\hline $\mathrm{Si}$ & o -12.77025300 & -1.82127500 & 0.48308600 \\
\hline $\mathrm{Si}$ & o -10.60122200 & -8.30452000 & 4.79669400 \\
\hline $\mathrm{Si}$ & o $\quad-8.83869200$ & -2.22196700 & -5.65234900 \\
\hline $\mathrm{Si}$ & o $\quad-0.17400100$ & -0.07133800 & -3.46808000 \\
\hline $\mathrm{Si}$ & o -10.60183500 & -3.99258600 & 4.81447500 \\
\hline $\mathrm{Si}$ & o $\quad-8.44853100$ & -10.47690800 & 0.46250500 \\
\hline $\mathrm{Si}$ & $\begin{array}{ll}0 & -2.35628300\end{array}$ & -4.40159500 & -5.61590600 \\
\hline $\mathrm{H}$ & $\begin{array}{ll}-1 & -5.59981300\end{array}$ & -8.34221900 & 5.28393600 \\
\hline $\mathrm{O}$ & o -4.03470600 & o -9.11429500 & 3.43025300 \\
\hline $\mathrm{H}$ & $-1 \quad-3.36023100$ & -7.48455700 & 5.21210900 \\
\hline $\mathrm{O}$ & o -11.82543400 & -1.32022300 & -0.76939500 \\
\hline $\mathrm{H}$ & $\begin{array}{ll}-1 & -9.25739700\end{array}$ & $-7 \cdot 30244800$ & -4.27887500 \\
\hline $\mathrm{H}$ & $-1 \quad-4.05335500$ & 2.90496200 & -0.14438900 \\
\hline $\mathrm{H}$ & $-1 \quad-4.91373000$ & 2.98078500 & -2.38539300 \\
\hline $\mathrm{H}$ & $-1 \quad-9.26460100$ & 1.09432900 & -4.27220300 \\
\hline $\mathrm{O}$ & o $\quad-7.56115900$ & -3.10251200 & -6.15343900 \\
\hline $\mathrm{H}$ & $\begin{array}{ll}-1 & -5.85964900\end{array}$ & -4.81023600 & -6.77125800 \\
\hline $\mathrm{H}$ & $-1-7.63561900$ & -6.60953500 & 5.94179200 \\
\hline $\mathrm{H}$ & $\begin{array}{ll}-1 & -11.06377900\end{array}$ & -0.67566200 & 3.43612600 \\
\hline $\mathrm{H}$ & $\begin{array}{ll}-1 & 0.29824700\end{array}$ & 2.13373600 & -0.61422600 \\
\hline $\mathrm{H}$ & $-1 \quad-1.47118300$ & 2.79431600 & 0.86828700 \\
\hline $\mathrm{H}$ & $\begin{array}{ll}-1 & 0.22678900\end{array}$ & 1.27609600 & 1.62541400 \\
\hline $\mathrm{H}$ & $\begin{array}{ll}-1 & -5.59979500\end{array}$ & -10.96113700 & 2.66491300 \\
\hline $\mathrm{H}$ & $\begin{array}{ll}-1 & -3.36019900\end{array}$ & -10.88941700 & 1.80730900 \\
\hline $\mathrm{O}$ & o -10.06543700 & o -3.19204600 & o $\quad-5.17316700$ \\
\hline $\mathrm{H}$ & $-1-11.75714800$ & -4.80339200 & -4.27899900 \\
\hline
\end{tabular}




$\begin{array}{lllll}\mathrm{H} & -1 & -13.64368000 & -2.91657600 & 0.07190700 \\ \mathrm{H} & -1 & -13.56786400 & -0.67555100 & 0.93221600 \\ \mathrm{H} & -1 & -10.13959100 & -9.11347300 & 5.94186700 \\ \mathrm{H} & -1 & -10.99998600 & -9.18927500 & 3.70086100 \\ \mathrm{H} & -1 & -11.75709800 & -7.49127200 & 5.21910200 \\ \mathrm{H} & -1 & -9.26455000 & -1.40503800 & -6.77151900 \\ \mathrm{H} & -1 & 0.95624500 & 0.40104300 & -2.65002100 \\ \mathrm{H} & -1 & -0.62417600 & 1.02320000 & -4.34367400 \\ \mathrm{H} & -1 & 0.23351600 & -1.21646100 & -4.27224500 \\ \mathrm{H} & -1 & -11.06387100 & -3.18124200 & 5.94181700 \\ \mathrm{H} & -1 & -11.68610400 & -4.87491200 & 4.36147000 \\ \mathrm{H} & -1 & -7.63561900 & -11.61908200 & 0.93225100 \\ \mathrm{H} & -1 & -9.32929700 & -10.03869200 & 1.55438500 \\ \mathrm{H} & -1 & -9.25777700 & -10.89634100 & -0.68523300 \\ \mathrm{H} & -1 & -1.54942200 & -5.53290000 & -6.07823900 \\ \mathrm{H} & -1 & -3.24304300 & -3.95252100 & -6.70034000 \\ \mathrm{H} & -1 & -1.47366900 & -3.29196300 & -5.21786600 \\ \mathrm{H} & \mathrm{o} & -4.07884700 & -4.08487000 & -0.14762900 \\ \mathrm{O} & \mathrm{o} & -4.74921500 & -3.34749700 & -0.16040000 \\ \mathrm{C} & \mathrm{o} & -2.85753700 & -5.61450600 & 0.46846400 \\ \mathrm{C} & \mathrm{o} & -2.30594800 & -5.12443300 & -0.66602500 \\ \mathrm{C} & \mathrm{o} & -1.58901000 & -3.87692600 & -0.36111000 \\ \mathrm{C} & \mathrm{o} & -1.69864300 & -3.60873800 & 0.95816900 \\ \mathrm{C} & \mathrm{o} & -2.49716400 & -4.70749900 & 1.61213000 \\ \mathrm{H} & \mathrm{o} & -3.43439700 & -6.52748700 & 0.56257800 \\ \mathrm{H} & \mathrm{o} & -2.38051200 & -5.57652700 & -1.64934800 \\ \mathrm{H} & \mathrm{o} & -1.07976800 & -3.26094500 & -1.09540400 \\ \mathrm{H} & \mathrm{o} & -1.89594300 & -5.24145900 & 2.36127800 \\ \mathrm{H} & \mathrm{o} & -3.38487200 & -4.33841700 & 2.14421800 \\ \mathrm{C} & \mathrm{o} & -1.18041200 & -2.42086400 & 1.69965200 \\ \mathrm{H} & \mathrm{o} & -0.53283100 & -1.81235700 & 1.06561500 \\ \mathrm{H} & \mathrm{o} & -2.00862300 & -1.78406000 & 2.03084000 \\ \mathrm{H} & \mathrm{o} & -0.62136000 & -2.72279400 & 2.59118400 \\ & & & & \end{array}$

\section{F-L* TS (H-FAU)}

(Electronic Energy: -10882.8579784 Ha):
$\mathrm{O}$
$\begin{array}{llll}\text { o } & -1.46357300 & 0.36133700 & 0.00022600\end{array}$
$\mathrm{O}$
$\begin{array}{llll}\text { o } & -4.96183300 & -9.22183000 & 0.85659700\end{array}$
$\mathrm{O}$
$\begin{array}{llll}\text { o } & -4.89132400 & -5.54767700 & -2.38460700\end{array}$
$\mathrm{O}$
$\begin{array}{llll}\text { o } & -7.74670600 & -0.29745400 & -0.07866200\end{array}$
$\begin{array}{llll}\text { o } & -4.97209800 & -6.55655300 & 3.60953000\end{array}$
$\begin{array}{llll}0 & -10.02680100 & -0.49606600 & -2.48511300\end{array}$
$\begin{array}{llll}\text { o } & -7.71340200 & -5.61972900 & -5.27460100 \\ \text { o } & -6.98000600 & -4.88096600 & -0.84582400\end{array}$
$\mathrm{O}$
$\begin{array}{llllll}\mathrm{O} & \mathrm{O} & -6.98000600 & -4.88096600 & -0.84582400 \\ \mathrm{O} & \mathrm{o} & -9.33298500 & -2.34543200 & -0.70095700\end{array}$
$\begin{array}{lllll}\mathrm{O} & \mathrm{o} & -9.38338500 & -4.97004400 & -3.29510700\end{array}$
$\begin{array}{lllll}\mathrm{O} & \mathrm{O} & -3.11023700 & 1.31704100 & -1.87315400\end{array}$
$\begin{array}{lllll}\mathrm{O} & \mathrm{o} & -11.83147800 & -2.38157000 & 1.71632900\end{array}$
$\begin{array}{lllll}\mathrm{O} & \mathrm{o} & -6.70082000 & -7.48254300 & 1.81702100\end{array}$
$\begin{array}{llllll}\mathrm{O} & \mathrm{o} & -9.37900000 & -7.34108800 & 4.24650000\end{array}$
$\begin{array}{lllll}\mathrm{O} & \mathrm{O} & -8.34530300 & -1.28134700 & -4.38879400\end{array}$ 


\begin{tabular}{|c|c|c|c|}
\hline $\mathrm{O}$ & o $\quad-5.79940800$ & -3.99808200 & -4.36533100 \\
\hline $\mathrm{O}$ & o $\quad-5.94361900$ & -1.44699000 & -1.67614000 \\
\hline $\mathrm{O}$ & o $\quad-7.30578400$ & -6.62492800 & -2.83356100 \\
\hline $\mathrm{O}$ & o $\quad-7.30516500$ & -2.69877700 & 1.01382700 \\
\hline $\mathrm{O}$ & o -11.02412900 & -2.98323500 & -2.65787500 \\
\hline $\mathrm{O}$ & o $\quad-1.39542000$ & -0.60778900 & -2.49424200 \\
\hline $\mathrm{O}$ & o -10.09426800 & -3.02093100 & 3.58837000 \\
\hline $\mathrm{O}$ & o $\quad-7.47605500$ & -5.65053700 & 3.57157100 \\
\hline $\mathrm{O}$ & o $\quad-7.48396000$ & -9.23459200 & -0.02294700 \\
\hline $\mathrm{O}$ & o $\quad-7.50307800$ & 0.48584100 & -2.60727400 \\
\hline $\mathrm{O}$ & o $\quad-4.01082300$ & -3.01312300 & -2.68221000 \\
\hline $\mathrm{O}$ & o $\quad-3.26153600$ & -4.92241800 & -4.32509600 \\
\hline $\mathrm{O}$ & o $\quad-5.81964100$ & $-7 \cdot 32498000$ & -0.75033700 \\
\hline $\mathrm{O}$ & o $\quad-9.41019900$ & -1.21793300 & 1.70611000 \\
\hline $\mathrm{O}$ & o $\quad-5.66708400$ & -4.99614900 & 1.68968100 \\
\hline $\mathrm{O}$ & o $\quad-5.60161900$ & 1.06496800 & -0.84941000 \\
\hline $\mathrm{O}$ & $\begin{array}{ll}\text { o } & -9.33615400\end{array}$ & -4.89521300 & $5 \cdot 33464500$ \\
\hline $\mathrm{O}$ & $0 \quad-3.41120900$ & -1.14362200 & -0.88771400 \\
\hline $\mathrm{Si}$ & o $\quad-6.29904800$ & -6.06145000 & -1.67664900 \\
\hline $\mathrm{Si}$ & o $\quad-8.41803400$ & -1.68535000 & 0.50190100 \\
\hline $\mathrm{Si}$ & o $\quad-4.50948800$ & -7.88565200 & 4.43121900 \\
\hline $\mathrm{Si}$ & o -10.54105600 & -1.79911000 & -1.64410000 \\
\hline $\mathrm{Al}$ & o $\quad-6.27112500$ & -3.94921500 & 0.45058100 \\
\hline $\mathrm{Si}$ & о $\quad-8.44100800$ & -6.16392000 & -3.90536500 \\
\hline $\mathrm{Si}$ & o $\quad-4.43991300$ & 2.12344600 & -1.31223100 \\
\hline $\mathrm{Si}$ & o $\quad-6.22996900$ & -6.13370100 & 2.64350100 \\
\hline $\mathrm{Si}$ & o $\quad-8.80595300$ & -0.01662300 & -3.45961800 \\
\hline $\mathrm{Si}$ & o $\quad-6.70370300$ & -4.39961100 & -5.67102600 \\
\hline $\mathrm{Si}$ & o $\quad-4.54094700$ & -2.20783700 & -1.35751500 \\
\hline $\mathrm{Si}$ & o $\quad-2.34028800$ & -0.01359400 & -1.31909100 \\
\hline $\mathrm{Si}$ & o $\quad-6.26245300$ & -8.29383100 & 0.46835400 \\
\hline $\mathrm{Si}$ & o $\quad-8.42280600$ & -6.13912300 & 4.80333000 \\
\hline $\mathrm{Si}$ & o -10.60618800 & -1.79869300 & 2.63951800 \\
\hline $\mathrm{Si}$ & o $\quad-4.51497600$ & $-4 \cdot 37087300$ & -3.44507100 \\
\hline $\mathrm{Si}$ & o $\quad-6.70146300$ & -0.04315300 & -1.28941800 \\
\hline $\mathrm{Si}$ & o $\quad-0.58608600$ & 1.68355300 & 0.47886500 \\
\hline $\mathrm{Si}$ & o -4.51397700 & -10.08850100 & 2.18437800 \\
\hline $\mathrm{Si}$ & o -10.59419500 & -4.01255700 & -3.84824800 \\
\hline $\mathrm{Si}$ & o -12.76845000 & -1.82266200 & 0.48665400 \\
\hline $\mathrm{Si}$ & o -10.60034500 & -8.30368200 & 4.79637500 \\
\hline $\mathrm{Si}$ & o $\quad-8.83127400$ & -2.20941000 & -5.64205700 \\
\hline $\mathrm{Si}$ & o -0.17414600 & -0.06949400 & -3.46876800 \\
\hline $\mathrm{Si}$ & o -10.60156800 & -3.98946200 & 4.81081400 \\
\hline $\mathrm{Si}$ & o $\quad-8.45148900$ & -10.47938100 & 0.46446400 \\
\hline $\mathrm{Si}$ & o $\quad-2.35786100$ & -4.40074700 & -5.61399600 \\
\hline $\mathrm{H}$ & $-1 \quad-5.59981300$ & -8.34221900 & 5.28393600 \\
\hline $\mathrm{O}$ & o $\quad-4.04559600$ & -9.07496500 & 3.3919780o \\
\hline $\mathrm{H}$ & $-1 \quad-3.36023100$ & -7.48455700 & 5.21210900 \\
\hline $\mathrm{O}$ & o -11.82019800 & -1.32304700 & -0.76180000 \\
\hline $\mathrm{H}$ & $-1 \quad-9.25739700$ & $-7 \cdot 30244800$ & -4.27887500 \\
\hline $\mathrm{H}$ & $-1-4.05335500$ & 2.90496200 & -0.14438900 \\
\hline $\mathrm{H}$ & $-1 \quad-4.91373000$ & 2.98078500 & -2.38539300 \\
\hline
\end{tabular}




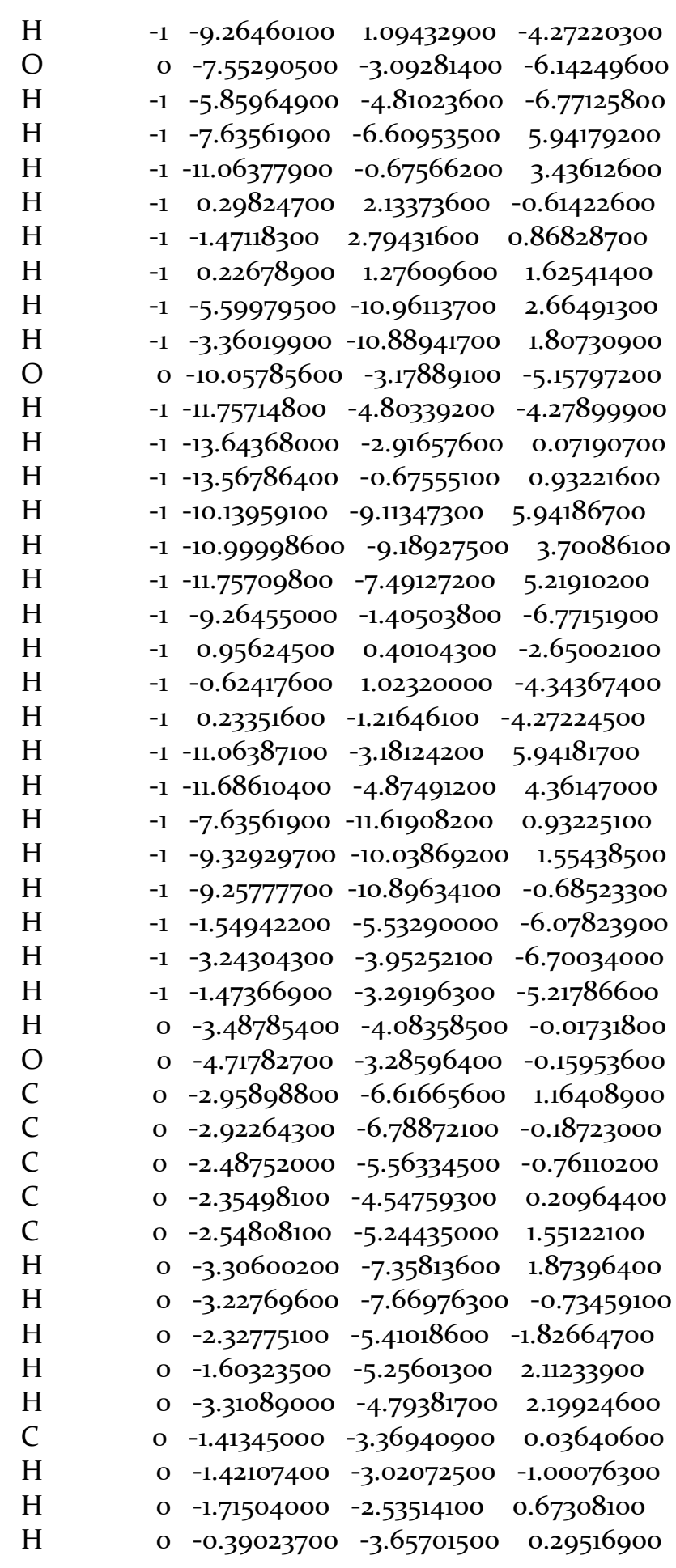

G-B* TS (H-FAU)

(Electronic Energy: -10882.8298774 Ha):
$\mathrm{O}$
$\begin{array}{lllll}\text { o } & -1.81640200 & 1.23293800 & 0.01665900\end{array}$
$\mathrm{O}$
$\begin{array}{lllll}\text { o } & -5.31163800 & -8.40107000 & 0.84548600\end{array}$
$\mathrm{O}$
$\begin{array}{lllll}\text { o } & -5.32898700 & -4.93681200 & -2.63759200\end{array}$
$\mathrm{O}$
$\begin{array}{llll}\text { o } & -7.80991800 & 0.34231400 & 0.08891800\end{array}$ 
$\begin{array}{llll}\text { o } & -5.30895800 & -5.67568800 & 3.61501400\end{array}$

$\begin{array}{llllll}\mathrm{O} & 0 & -10.50368100 & 0.28939300 & -2.64949000\end{array}$

$\begin{array}{lllll}\mathrm{O} & \mathrm{O} & -7.85073500 & -4.81963900 & -5.10109000\end{array}$

$\begin{array}{lllll}\mathrm{O} & \mathrm{o} & -7.19384800 & -3.91140600 & -1.05814300\end{array}$

$\begin{array}{lllll}\mathrm{O} & \mathrm{O} & -9.62215900 & -1.38628100 & -0.78685700\end{array}$

$\mathrm{O}$

$\begin{array}{llll}\text { o } & -9.70121300 & -4.10465500 & -3.32836000\end{array}$

$\begin{array}{llll}\text { o } & -3.50600900 & 2.09460100 & -1.84732300\end{array}$

$\begin{array}{llll}\text { o } & -12.18276700 & -1.54113400 & 1.73295400\end{array}$

$\begin{array}{llll}\text { o } & -7.06408000 & -6.74140400 & 1.90633600\end{array}$

$\begin{array}{llll}\text { o } & -9.71873800 & -6.47788900 & 4.24666500\end{array}$

$\begin{array}{llll}\text { o } & -8.60947400 & -0.38558500 & -4.41357400\end{array}$

$\begin{array}{llll}\text { o } & -6.01548500 & -3.01482300 & -4.39211800\end{array}$

$\begin{array}{llll}\text { o } & -6.12260100 & -0.49641800 & -1.82913300\end{array}$

$\begin{array}{llll}\text { o } & -7.81610000 & -5.92020800 & -2.65190500\end{array}$

$\begin{array}{llll}\text { o } & -7.92217300 & -2.12654100 & 1.14267700\end{array}$

$\begin{array}{llll}\text { o } & -11.41174600 & -2.22705400 & -2.56634300\end{array}$

$\begin{array}{lllll}0 & -1.71526500 & 0.26362300 & -2.48838100\end{array}$

$\begin{array}{llll}\text { o } & -10.41387000 & -2.15401200 & 3.58950400\end{array}$

$\begin{array}{llll}\text { o } & -7.80782400 & -4.79225000 & 3.58195100\end{array}$

$\begin{array}{llll}\text { o } & -7.82569400 & -8.37324500 & -0.04396600\end{array}$

$\begin{array}{lllll}\text { o } & -8.00383000 & 1.27575300 & -2.42934600\end{array}$

$\begin{array}{llll}\text { o } & -4.38368200 & -2.41343100 & -2.39802900\end{array}$

$\begin{array}{llll}\text { o } & -3.55948500 & -4.04919000 & -4.31425000\end{array}$

$\begin{array}{llll}\text { o } & -6.17420100 & -6.38266100 & -0.62211500\end{array}$

$\begin{array}{llll}\text { o } & -9.78563700 & -0.33779900 & 1.67225400\end{array}$

$\begin{array}{lllll}\text { o } & -6.11416300 & -4.24504900 & 1.60718300\end{array}$

$\begin{array}{lllll}0 & -6.00203300 & 1.97123000 & -0.82319500\end{array}$

$\begin{array}{llll}\text { o } & -9.66155800 & -4.03014700 & 5.33710700\end{array}$

$\begin{array}{llll}0 & -3.66014200 & -0.38857500 & -0.82413000\end{array}$

$\begin{array}{llll}\text { o } & -6.64523900 & -5.26787700 & -1.71619600\end{array}$

$\begin{array}{llll}\text { o } & -8.76922500 & -0.91852800 & 0.53514500\end{array}$

$\begin{array}{llll}\text { o } & -4.83755300 & -7.02839600 & 4.41536400\end{array}$

$\begin{array}{llll}0 & -10.91371500 & -0.95587900 & -1.67792900\end{array}$

$\begin{array}{lllll}\text { Al } & 0 & -6.71954300 & -3.14118200 & 0.41324400\end{array}$

Si $\quad \begin{array}{lllll}\quad & 0 & -8.76273100 & -5.34667100 & -3.84414900\end{array}$

$\begin{array}{lllll}\mathrm{Si} & 0 & -4.79601100 & 2.96877400 & -1.30885600\end{array}$

$\begin{array}{lllll}\mathrm{Si} & 0 & -6.59551600 & -5.34899600 & 2.64257200\end{array}$

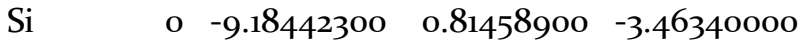

$\begin{array}{llllll}\mathrm{Si} & 0 & -6.96845400 & -3.54297800 & -5.61509900\end{array}$

Si $\quad \begin{array}{lllll} & 0 & -4.88295400 & -1.39681900 & -1.23140100\end{array}$

$\begin{array}{llllll}\mathrm{Si} & & 0 & -2.67569200 & 0.79258400 & -1.30483000\end{array}$

$\begin{array}{lllll}\mathrm{Si} & 0 & -6.60313000 & -7.46115200 & 0.50018600\end{array}$

$\begin{array}{llllll}\mathrm{Si} & & \mathrm{o} & -8.75886900 & -5.28502800 & 4.80681900\end{array}$

$\begin{array}{lllll}\mathrm{Si} & 0 & -10.95064200 & -0.94517700 & 2.63573700\end{array}$

$\begin{array}{llllll}\mathrm{Si} & 0 & -4.83436200 & -3.60142300 & -3.42132500\end{array}$

$\begin{array}{llllll}\mathrm{Si} & & 0 & -6.99981300 & 0.75624000 & -1.25942500\end{array}$

$\begin{array}{llllll}\mathrm{Si} & 0 & -0.91594700 & 2.54300600 & 0.47040100\end{array}$

$\begin{array}{lllll}\mathrm{Si} & 0 & -4.84153600 & -9.23594200 & 2.17993700\end{array}$

Si $\quad \begin{array}{lllll}\quad & 0 & -10.93887600 & -3.15891700 & -3.82431600\end{array}$

$\begin{array}{lllll}\mathrm{Si} & \mathrm{o} & -13.10339000 & -0.97099400 & 0.49291200\end{array}$

$\begin{array}{lllll}\mathrm{Si} & \mathrm{o} & -10.93281200 & -7.45100700 & 4.79377000\end{array}$

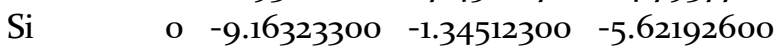




\begin{tabular}{|c|c|c|c|}
\hline $\mathrm{Si}$ & o $\quad-0.50009100$ & 0.78558500 & -3.47397800 \\
\hline $\mathrm{Si}$ & o -10.92942100 & -3.13207300 & 4.80575700 \\
\hline $\mathrm{Si}$ & o $\quad-8.78006400$ & -9.62271100 & 0.45811300 \\
\hline $\mathrm{Si}$ & $\begin{array}{ll}0 & -2.68300900\end{array}$ & -3.54498200 & -5.61454800 \\
\hline $\mathrm{H}$ & $-1 \quad-5.92888400$ & -7.48689500 & o $\quad 5.28067300$ \\
\hline $\mathrm{O}$ & o $\quad-4.38495200$ & -8.21976300 & 3.38846100 \\
\hline $\mathrm{H}$ & $\begin{array}{ll}-1 & -3.68930800\end{array}$ & -6.62922700 & o $\quad 5.20876700$ \\
\hline $\mathrm{O}$ & o -12.14100700 & -0.46056300 & -0.73756600 \\
\hline $\mathrm{H}$ & $-1 \quad-9.58683500$ & -6.44734800 & o -4.28222400 \\
\hline $\mathrm{H}$ & $\begin{array}{ll}-1 & -4.38241300\end{array}$ & 3.76037500 & -0.14768500 \\
\hline $\mathrm{H}$ & $-1 \quad-5.24280900$ & 3.83615100 & -2.38869800 \\
\hline $\mathrm{H}$ & $-1 \quad-9.59359800$ & 1.94956300 & -4.27546900 \\
\hline $\mathrm{O}$ & o $\quad-7.94742800$ & -2.32380800 & o $\quad-6.09095500$ \\
\hline $\mathrm{H}$ & $-1 \quad-6.18850400$ & -3.95480400 & -6.77479000 \\
\hline $\mathrm{H}$ & $\begin{array}{ll}-1 & -7.96466600\end{array}$ & -5.75419100 & 5.93849900 \\
\hline $\mathrm{H}$ & $-1-11.39297600$ & 0.17976300 & 3.43289600 \\
\hline $\mathrm{H}$ & $\begin{array}{ll}-1 & -0.03079400\end{array}$ & 2.98910000 & -0.61753400 \\
\hline $\mathrm{H}$ & $-1-1.80023800$ & 3.64971000 & o.8649870o \\
\hline $\mathrm{H}$ & $-1-0.10226600$ & 2.13145800 & 1.62212700 \\
\hline $\mathrm{H}$ & $-1 \quad-5.92889300$ & -10.10592400 & 2.66168800 \\
\hline $\mathrm{H}$ & $-1 \quad-3.68929700$ & -10.03408300 & $0 \quad 1.80403200$ \\
\hline $\mathrm{O}$ & o -10.43813400 & -2.21454000 & -5.07024000 \\
\hline $\mathrm{H}$ & $-1-12.08611400$ & -3.94807100 & -4.28222800 \\
\hline $\mathrm{H}$ & $-1-13.97270600$ & -2.06124000 & 0.06859700 \\
\hline $\mathrm{H}$ & $-1-13.89693100$ & o.1797780o & 0.92890400 \\
\hline $\mathrm{H}$ & $-1-10.46865800$ & -8.25813100 & 5.93855600 \\
\hline $\mathrm{H}$ & $-1-11.32905300$ & -8.33392800 & 3.69755300 \\
\hline $\mathrm{H}$ & $-1-12.08616800$ & -6.63593400 & 5.21579800 \\
\hline $\mathrm{H}$ & $-1 \quad-9.59358800$ & -0.54971500 & -6.77478000 \\
\hline $\mathrm{H}$ & $-1 \quad 0.62719600$ & 1.25639900 & -2.65333300 \\
\hline $\mathrm{H}$ & $-1 \quad-0.95322800$ & 1.87855200 & -4.34700200 \\
\hline $\mathrm{H}$ & $-1 \quad-0.09555500$ & -0.36111600 & -4.27554000 \\
\hline $\mathrm{H}$ & $-1 \quad-11.39296800$ & -2.32589400 & 5.93853700 \\
\hline $\mathrm{H}$ & $-1-12.01514200$ & -4.01956000 & $4 \cdot 35816200$ \\
\hline $\mathrm{H}$ & $-1 \quad-7.96471000$ & -10.76378400 & 0.92897100 \\
\hline $\mathrm{H}$ & $-1-9.65843200$ & -9.18338400 & 1.55110500 \\
\hline $\mathrm{H}$ & $-1-9.58687200$ & -10.04103700 & -0.68855200 \\
\hline $\mathrm{H}$ & $-1 \quad-1.87843000$ & -4.67754300 & -6.08164100 \\
\hline $\mathrm{H}$ & $-1-3.57211900$ & -3.09713600 & -6.70375200 \\
\hline $\mathrm{H}$ & $-1-1.80267100$ & -2.43652800 & -5.22125500 \\
\hline $\mathrm{O}$ & o $\quad-5.29424200$ & -2.12671300 & 0.14136400 \\
\hline $\mathrm{C}$ & o -3.71017900 & -2.88209000 & 2.52322900 \\
\hline $\mathrm{C}$ & o $\quad-2.44634100$ & -2.09239300 & 2.35711800 \\
\hline $\mathrm{C}$ & o $\quad-2.71042500$ & -0.78352200 & 2.28399200 \\
\hline $\mathrm{C}$ & o $\quad-4.18240700$ & -0.56331200 & 2.45667900 \\
\hline $\mathrm{C}$ & o $\quad-4.76936300$ & -1.85642400 & 2.50029400 \\
\hline $\mathrm{H}$ & o $\quad-3.93795700$ & -3.52026900 & 1.65380800 \\
\hline $\mathrm{H}$ & o $\quad-3.78417300$ & -3.56729200 & 3.37809900 \\
\hline $\mathrm{H}$ & o $\quad-1.46909100$ & -2.55165100 & 2.28451300 \\
\hline $\mathrm{H}$ & $0 \quad-2.01364200$ & 0.02565000 & 2.11606300 \\
\hline $\mathrm{H}$ & o $\quad-4.38662600$ & -0.77636800 & 3.60384700 \\
\hline 11 & o $\quad-5.83248700$ & -2.03493200 & 2.64888200 \\
\hline
\end{tabular}




$\begin{array}{lllll}\mathrm{C} & \mathrm{o} & -4.91884300 & 0.68478900 & 2.04746000 \\ \mathrm{H} & \mathrm{o} & -4.82951700 & 0.77744800 & 0.96427600 \\ \mathrm{H} & \mathrm{o} & -5.98198900 & 0.62555000 & 2.28830900 \\ \mathrm{H} & \mathrm{o} & -4.48135400 & 1.57090800 & 2.51112100\end{array}$

\section{G-L* TS (H-FAU)}

(Electronic Energy: -10882.8761939 Ha):
$\mathrm{O}$
$\begin{array}{llll}\text { o } & -1.81079500 & 1.23635000 & 0.00081700\end{array}$
$\mathrm{O}$
$\begin{array}{lllll}\text { o } & -5.32198400 & -8.36643500 & 0.86253300\end{array}$
$\mathrm{O}$
$\begin{array}{lllll}\text { o } & -5.23661000 & -4.85330100 & -2.52286800\end{array}$
$\begin{array}{llllll}\mathrm{O} & \mathrm{O} & -8.10280100 & 0.60761900 & -0.07787000\end{array}$
$\begin{array}{lllll}\mathrm{O} & \mathrm{O} & -5.32993900 & -5.69634200 & 3.59183000\end{array}$
$\begin{array}{lllll}\mathrm{O} & \mathrm{o} & -10.33320800 & 0.38096100 & -2.46373800\end{array}$
$\begin{array}{lllll}\mathrm{O} & \mathrm{o} & -7.96306400 & -4.79617300 & -5.19424300\end{array}$
$\begin{array}{llllll}\mathrm{O} & \mathrm{O} & -7.13089900 & -3.81047700 & -1.03544600\end{array}$
$\begin{array}{lllll}\mathrm{O} & \mathrm{o} & -9.65896100 & -1.47671600 & -0.68647900\end{array}$
$\begin{array}{lllll}\mathrm{O} & \mathrm{O} & -9.71795800 & -4.10234100 & -3.30828300\end{array}$
$\begin{array}{llllll}\mathrm{O} & \mathrm{o} & -3.40855800 & 2.20006000 & -1.89077400\end{array}$
$\begin{array}{lllll}\mathrm{O} & \mathrm{o} & -12.15572200 & -1.52702500 & 1.71000700\end{array}$
$\begin{array}{lllll}\mathrm{O} & \mathrm{o} & -7.11041000 & -6.72104800 & 1.90670200\end{array}$
$\begin{array}{lllll}\mathrm{O} & \mathrm{o} & -9.72411200 & -6.48092600 & 4.25054000\end{array}$
$\begin{array}{lllll}\mathrm{O} & \mathrm{o} & -8.70745600 & -0.45466800 & -4.39684600\end{array}$
$\begin{array}{lllll}\mathrm{O} & \mathrm{o} & -6.09066500 & -3.09376800 & -4.37842200\end{array}$
$\begin{array}{lllll}\mathrm{O} & \mathrm{O} & -6.29522400 & -0.71865500 & -1.47033300\end{array}$
$\begin{array}{llllll}\mathrm{O} & \mathrm{o} & -7.73709900 & -5.79815400 & -2.70673400\end{array}$
$\begin{array}{lllll}\mathrm{O} & \mathrm{o} & -7.60804800 & -1.77073300 & 1.01525400\end{array}$
$\begin{array}{lllll}\mathrm{O} & \mathrm{O} & -11.33222000 & -2.10544700 & -2.67273600\end{array}$
$\begin{array}{llllll}\mathrm{O} & 0 & -1.71201100 & 0.25219600 & -2.50285000\end{array}$
$\begin{array}{lllll}\mathrm{O} & \mathrm{o} & -10.42146600 & -2.17085100 & 3.58794600\end{array}$
$\begin{array}{llllll}\mathrm{O} & \mathrm{O} & -7.84245600 & -4.78376000 & 3.59659200\end{array}$
$\begin{array}{lllll}\mathrm{O} & \mathrm{o} & -7.82586700 & -8.36932400 & -0.05028400\end{array}$
$\begin{array}{llllll}\mathrm{O} & \mathrm{O} & -7.76372000 & 1.23082300 & -2.63650000\end{array}$
$\begin{array}{lllll}\mathrm{O} & \mathrm{O} & -4.39571700 & -2.28159500 & -2.56251800\end{array}$
$\begin{array}{lllll}\mathrm{O} & \mathrm{o} & -3.57632500 & -4.05164100 & -4.33878200\end{array}$
$\begin{array}{lllll}\mathrm{O} & \mathrm{O} & -6.21455300 & -6.33831300 & -0.60367000\end{array}$
$\begin{array}{lllll}\mathrm{O} & \mathrm{o} & -9.74159000 & -0.34818500 & 1.72180300\end{array}$
$\begin{array}{lllll}\mathrm{O} & \mathrm{O} & -6.20059000 & -4.21892100 & 1.62911400\end{array}$
$\begin{array}{lllll}\mathrm{O} & \mathrm{O} & -5.88621500 & 1.86537500 & -0.88591200\end{array}$
$\begin{array}{lllll}\mathrm{O} & \mathrm{O} & -9.68333100 & -4.04201900 & 5.36538600\end{array}$
$\begin{array}{llllll}\mathrm{O} & 0 & -3.76143800 & -0.24632400 & -0.94349200\end{array}$
Si $\quad \begin{array}{lllll}0 & 0 & -6.61660700 & -5.18967500 & -1.68610800\end{array}$
$\begin{array}{llllll}\mathrm{Si} & 0 & -8.75716000 & -0.78708700 & 0.50794500\end{array}$
Si $\quad \begin{array}{lllll} & 0 & -4.83817100 & -7.02535400 & 4.41588400\end{array}$
$\begin{array}{lllll}\mathrm{Si} & 0 & -10.86179300 & -0.93135800 & -1.64423200\end{array}$
$\begin{array}{lllll}\mathrm{Al} & \mathrm{o} & -6.65895400 & -3.07201200 & 0.45385400\end{array}$
$\begin{array}{lllll}\mathrm{Si} & 0 & -8.77495900 & -5.32073600 & -3.86662200\end{array}$
$\begin{array}{lllll}\mathrm{Si} & 0 & -4.75485900 & 2.97374400 & -1.32387700\end{array}$
$\begin{array}{lllll}\mathrm{Si} & 0 & -6.63209100 & -5.34059000 & 2.66727700\end{array}$
Si $\quad \begin{array}{lllll} & 0 & -9.12035900 & 0.82235300 & -3.46746800\end{array}$
Si $\quad \begin{array}{lllll} & 0 & -7.01176700 & -3.55211500 & -5.65364900\end{array}$
Si $\quad \begin{array}{lllll}0 & -4.82647500 & -1.38790400 & -1.29385200\end{array}$

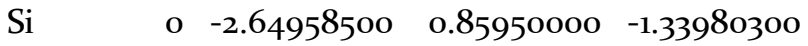




\begin{tabular}{|c|c|c|c|}
\hline $\mathrm{Si}$ & o $\quad-6.62361800$ & -7.43964300 & 0.51400600 \\
\hline $\mathrm{Si}$ & o $\quad-8.77560100$ & -5.28630100 & 4.82624700 \\
\hline $\mathrm{Si}$ & o -10.93926800 & -0.94718100 & 2.64533000 \\
\hline $\mathrm{Si}$ & o $\quad-4.84491400$ & $-3 \cdot 5^{8} 517900$ & -3.45547500 \\
\hline $\mathrm{Si}$ & o $\quad-7.01180100$ & 0.76193700 & -1.26689000 \\
\hline $\mathrm{Si}$ & $0-0.91385200$ & 2.54657800 & 0.47687600 \\
\hline $\mathrm{Si}$ & o $\quad-4.84216100$ & -9.22828600 & 2.18347800 \\
\hline $\mathrm{Si}$ & o -10.92957000 & -3.15094500 & -3.86080400 \\
\hline $\mathrm{Si}$ & o -13.09695100 & -0.96706000 & 0.48290300 \\
\hline $\mathrm{Si}$ & o -10.93640300 & -7.45373200 & 4.79604700 \\
\hline $\mathrm{Si}$ & o $\quad-9.17869000$ & -1.37763800 & -5.65802800 \\
\hline $\mathrm{Si}$ & o $\quad-0.49317300$ & 0.78784900 & $-3.4795^{2200}$ \\
\hline $\mathrm{Si}$ & o -10.93457600 & -3.13653700 & 4.81431200 \\
\hline $\mathrm{Si}$ & o $\quad-8.77874000$ & -9.62108200 & 0.45780400 \\
\hline $\mathrm{Si}$ & o $\quad-2.67821300$ & $-3 \cdot 54264800$ & -5.62593600 \\
\hline $\mathrm{H}$ & $\begin{array}{ll}-1 & -5.92887300\end{array}$ & -7.48688100 & 5.28066200 \\
\hline $\mathrm{O}$ & o $\quad-4.37962700$ & -8.22625500 & 3.39771600 \\
\hline $\mathrm{H}$ & $\begin{array}{ll}-1 & -3.68929800\end{array}$ & -6.62923500 & 5.20876100 \\
\hline $\mathrm{O}$ & o -12.14659100 & -0.46648900 & -0.76513200 \\
\hline $\mathrm{H}$ & $\begin{array}{ll}-1 & -9.58663100\end{array}$ & -6.44725000 & -4.28226300 \\
\hline $\mathrm{H}$ & $\begin{array}{ll}-1 & -4.38239200\end{array}$ & 3.76037100 & -0.14770300 \\
\hline $\mathrm{H}$ & $\begin{array}{ll}-1 & -5.24282000\end{array}$ & 3.83622700 & -2.38871900 \\
\hline $\mathrm{H}$ & $\begin{array}{ll}-1 & -9.59362500\end{array}$ & 1.94960700 & -4.27550700 \\
\hline $\mathrm{O}$ & o $\quad-7.90720300$ & -2.27898500 & -6.13660800 \\
\hline $\mathrm{H}$ & $-1 \quad-6.18869400$ & -3.95485800 & -6.77468000 \\
\hline $\mathrm{H}$ & $-1 \quad-7.96465600$ & $-5 \cdot 75418900$ & $5 \cdot 93849300$ \\
\hline $\mathrm{H}$ & $-1-11.39299200$ & 0.17975900 & 3.43290300 \\
\hline $\mathrm{H}$ & $-1 \quad-0.03082700$ & 2.98906600 & o -0.61750300 \\
\hline $\mathrm{H}$ & $-1 \quad-1.80021700$ & 3.64965300 & 0.86502500 \\
\hline $\mathrm{H}$ & $-1-0.10227400$ & 2.13140500 & 1.62210100 \\
\hline $\mathrm{H}$ & $\begin{array}{ll}-1 & -5.92888800\end{array}$ & -10.10590900 & o $\quad 2.66167700$ \\
\hline $\mathrm{H}$ & $\begin{array}{ll}-1 & -3.68930400\end{array}$ & -10.03406200 & o $\quad 1.80402800$ \\
\hline $\mathrm{O}$ & o -10.42060500 & -2.33367600 & o $\quad-5.19039400$ \\
\hline $\mathrm{H}$ & $-1-12.08611100$ & -3.94804400 & -4.28228800 \\
\hline $\mathrm{H}$ & $-1-13.97271500$ & -2.06124700 & 0.06859200 \\
\hline $\mathrm{H}$ & $\begin{array}{ll}-1 & -13.89692200\end{array}$ & 0.17976500 & 0.92890700 \\
\hline $\mathrm{H}$ & $-1-10.46865600$ & -8.25812400 & 5.93854900 \\
\hline $\mathrm{H}$ & $-1-11.32904800$ & -8.33392300 & 3.69755400 \\
\hline $\mathrm{H}$ & $-1-12.08616000$ & -6.63593300 & 5.21579400 \\
\hline $\mathrm{H}$ & $\begin{array}{ll}-1 & -9.59360100\end{array}$ & -0.54981500 & -6.77476600 \\
\hline $\mathrm{H}$ & $\begin{array}{ll}-1 & 0.62716900\end{array}$ & 1.25637900 & -2.65332900 \\
\hline $\mathrm{H}$ & $-1 \quad-0.95321600$ & 1.87855300 & -4.34700400 \\
\hline $\mathrm{H}$ & $\begin{array}{ll}-1 & -0.09555600\end{array}$ & -0.36112000 & -4.27554800 \\
\hline $\mathrm{H}$ & $\begin{array}{ll}-1 & -11.39296600\end{array}$ & -2.32589700 & 5.93853700 \\
\hline $\mathrm{H}$ & $\begin{array}{ll}-1 & -12.01512900\end{array}$ & -4.01955600 & 4.35815800 \\
\hline $\mathrm{H}$ & $\begin{array}{ll}-1 & -7.96471200\end{array}$ & -10.76376500 & 0.92896600 \\
\hline $\mathrm{H}$ & $-1 \quad-9.65842500$ & -9.18337900 & 1.55109900 \\
\hline $\mathrm{H}$ & $\begin{array}{ll}-1 & -9.58686100\end{array}$ & -10.04102500 & -0.68854700 \\
\hline $\mathrm{H}$ & $\begin{array}{ll}-1 & -1.87844700\end{array}$ & -4.67752600 & -6.08160500 \\
\hline $\mathrm{H}$ & $-1 \quad-3.57210400$ & -3.09714900 & -6.70372300 \\
\hline $\mathrm{H}$ & $\begin{array}{ll}-1 & -1.80267600\end{array}$ & -2.43652100 & -5.22125200 \\
\hline $\mathrm{O}$ & o $\quad-4.91412100$ & -2.40098100 & 0.06306700 \\
\hline
\end{tabular}




$\begin{array}{lllll}\mathrm{C} & \mathrm{o} & -2.74658900 & -3.65204200 & -0.24506800 \\ \mathrm{C} & \mathrm{o} & -1.64999000 & -2.61379300 & -0.31086500 \\ \mathrm{C} & \mathrm{o} & -1.74629400 & -1.74721600 & 0.69695900 \\ \mathrm{C} & \mathrm{o} & -2.93596900 & -2.04424600 & 1.58514800 \\ \mathrm{C} & \mathrm{o} & -3.74593800 & -3.05105300 & 0.74958500 \\ \mathrm{H} & \mathrm{o} & -3.19872600 & -3.93069600 & -1.19758100 \\ \mathrm{H} & \mathrm{o} & -2.35958500 & -4.58384200 & 0.18815000 \\ \mathrm{H} & \mathrm{o} & -0.86536000 & -2.63030500 & -1.05888100 \\ \mathrm{H} & \mathrm{o} & -1.05616900 & -0.93785800 & 0.90532300 \\ \mathrm{H} & \mathrm{o} & -2.56968900 & -2.63080100 & 2.44276300 \\ \mathrm{H} & \mathrm{o} & -4.22667700 & -3.79823300 & 1.36995700 \\ \mathrm{C} & \mathrm{o} & -3.71758100 & -0.86179800 & 2.15343900 \\ \mathrm{H} & \mathrm{o} & -4.05408000 & -0.18190800 & 1.37018700 \\ \mathrm{H} & \mathrm{o} & -4.59263700 & -1.21331200 & 2.70860700 \\ \mathrm{H} & \mathrm{o} & -3.08427100 & -0.29434900 & 2.84043800\end{array}$

\section{R-J* TS (H-FAU)}

(Electronic Energy: -10882.8136577 Ha):

\begin{tabular}{lllll}
$\mathrm{O}$ & $\mathrm{o}$ & -1.43710400 & 0.35546600 & -0.01328100 \\
$\mathrm{O}$ & $\mathrm{o}$ & -4.97197700 & -9.19709000 & 0.89450900 \\
$\mathrm{O}$ & $\mathrm{o}$ & -4.88784200 & -5.55056500 & -2.36688700 \\
$\mathrm{O}$ & $\mathrm{o}$ & -7.76626500 & -0.31622400 & -0.09264200 \\
$\mathrm{O}$ & $\mathrm{o}$ & -4.96984600 & -6.60189600 & 3.55231900 \\
$\mathrm{O}$ & $\mathrm{o}$ & -10.02961500 & -0.47591400 & -2.47112000 \\
$\mathrm{O}$ & $\mathrm{o}$ & -7.72025400 & -5.61608500 & -5.27505800 \\
$\mathrm{O}$ & $\mathrm{o}$ & -7.02248700 & -4.86910400 & -0.88105900 \\
$\mathrm{O}$ & $\mathrm{o}$ & -9.35875900 & -2.35539500 & -0.70684100 \\
$\mathrm{O}$ & $\mathrm{o}$ & -9.38288000 & -4.97313000 & -3.29086200 \\
$\mathrm{O}$ & $\mathrm{o}$ & -3.19294000 & 1.21850300 & -1.83344200 \\
$\mathrm{O}$ & $\mathrm{o}$ & -11.84130000 & -2.38491100 & 1.71686400 \\
$\mathrm{O}$ & $\mathrm{o}$ & -6.77675000 & -7.50181500 & 1.82280200 \\
$\mathrm{O}$ & $\mathrm{o}$ & -9.38501200 & -7.33682400 & 4.24898900 \\
$\mathrm{O}$ & $\mathrm{o}$ & -8.37357500 & -1.29721200 & -4.38006100 \\
$\mathrm{O}$ & $\mathrm{o}$ & -5.79457200 & -4.01605400 & -4.36295400 \\
$\mathrm{O}$ & $\mathrm{o}$ & -5.84879500 & -1.38959600 & -1.63153100 \\
$\mathrm{O}$ & $\mathrm{o}$ & -7.29607400 & -6.63867000 & -2.84016300 \\
$\mathrm{O}$ & $\mathrm{o}$ & -7.36208400 & -2.71106600 & 1.03289200 \\
$\mathrm{O}$ & $\mathrm{o}$ & -11.02065900 & -2.96232600 & -2.69169900 \\
$\mathrm{O}$ & $\mathrm{o}$ & -1.41175400 & -0.60591700 & -2.51299100 \\
$\mathrm{O}$ & $\mathrm{o}$ & -10.09672800 & -3.02016000 & 3.58557800 \\
$\mathrm{O}$ & $\mathrm{o}$ & -7.47155400 & -5.65226700 & 3.59366200 \\
$\mathrm{O}$ & $\mathrm{o}$ & -7.47601700 & -9.24268400 & -0.04824900 \\
$\mathrm{O}$ & $\mathrm{o}$ & -7.48639100 & 0.45579400 & -2.61704500 \\
$\mathrm{O}$ & $\mathrm{o}$ & -4.02575800 & -3.01065600 & -2.67847000 \\
$\mathrm{O}$ & $\mathrm{o}$ & -3.25413600 & -4.91680900 & -4.31336100 \\
$\mathrm{O}$ & $\mathrm{o}$ & -5.81422600 & -7.29074300 & -0.71004400 \\
$\mathrm{O}$ & $\mathrm{o}$ & -9.44190600 & -1.19824400 & 1.69329600 \\
$\mathrm{O}$ & $\mathrm{o}$ & -5.74247500 & -5.02463100 & 1.66427300 \\
$\mathrm{O}$ & $\mathrm{o}$ & -5.68031200 & 1.13192300 & -0.79703400 \\
$\mathrm{O}$ & $\mathrm{o}$ & -9.33972300 & -4.89333000 & 5.34178400 \\
$\mathrm{O}$ & $\mathrm{o}$ & -3.29407800 & -1.28455200 & -0.81166300 \\
$\mathrm{Si}$ & $\mathrm{o}$ & -6.30826400 & -6.05765500 & -1.67223300 \\
& & & & \\
\hline
\end{tabular}




\begin{tabular}{|c|c|c|c|c|}
\hline $\mathrm{Si}$ & o & -8.45232600 & -1.68882100 & 0.49774000 \\
\hline $\mathrm{Si}$ & o & -4.50163600 & -7.90109700 & 4.42168300 \\
\hline $\mathrm{S}$ & $\mathrm{o}$ & -10.55327400 & -1.79235200 & -1.65572500 \\
\hline $\mathrm{Al}$ & $\mathrm{o}$ & -6.33912300 & -3.95544200 & 0.44527900 \\
\hline $\mathrm{Si}$ & $\mathrm{o}$ & -8.43830700 & -6.16724700 & -3.90200800 \\
\hline S & $\mathrm{o}$ & -4.47423000 & 2.11620700 & -1.29521400 \\
\hline $\mathrm{S}$ & o & -6.25846500 & -6.16278900 & 2.63742300 \\
\hline S & o & -8.80781600 & -0.01973500 & -3.45610800 \\
\hline $\mathrm{Si}$ & o & -6.70237600 & -4.40239700 & -5.66861500 \\
\hline J1 & o & -4.50917800 & -2.26099300 & -1.30178900 \\
\hline $\mathrm{SI}$ & o & -2.34806500 & -0.07005200 & -1.29614400 \\
\hline $\mathrm{Si}$ & $\mathrm{o}$ & -6.27508900 & -8.29237700 & 0.48287100 \\
\hline $\mathrm{Si}$ & $\mathrm{o}$ & -8.42737400 & -6.13980200 & 4.81233300 \\
\hline $\mathrm{Si}$ & $\mathrm{o}$ & -10.61697400 & -1.79946500 & 2.63955000 \\
\hline $\mathrm{Si}$ & $\mathrm{o}$ & -4.51574800 & -4.37576500 & -3.42906400 \\
\hline $\mathrm{Si}$ & $\mathrm{o}$ & -6.69882900 & -0.03966700 & o $\quad-1.27586100$ \\
\hline $\mathrm{Si}$ & $\mathrm{o}$ & -0.58658100 & 1.68501300 & 0.47657900 \\
\hline $\mathrm{Si}$ & $\mathrm{o}$ & -4.50757800 & -10.08698300 & o $\quad 2.19982600$ \\
\hline $\mathrm{Si}$ & o & -10.58879900 & -4.01734100 & -3.85883700 \\
\hline $\mathrm{Si}$ & o & -12.77229400 & -1.82302000 & 0.48469400 \\
\hline $\mathrm{Si}$ & o & -10.60149300 & -8.30418900 & 4.79671300 \\
\hline $\mathrm{Si}$ & o & -8.83279700 & -2.21894300 & -5.64706200 \\
\hline $\mathrm{Si}$ & o & -0.18539000 & -0.06519800 & -3.46755500 \\
\hline $\mathrm{Si}$ & o & -10.60171500 & -3.98914100 & 4.81058300 \\
\hline $\mathrm{Si}$ & o & -8.44779200 & -10.47716000 & 0.46134800 \\
\hline $\mathrm{Si}$ & o & -2.36409100 & -4.40398000 & -5.61047400 \\
\hline $\mathrm{H}$ & -1 & $-5 \cdot 59982400$ & -8.34224300 & 5.28399600 \\
\hline $\mathrm{O}$ & o & -4.02317900 & -9.12443000 & 3.43712300 \\
\hline $\mathrm{H}$ & -1 & -3.36023200 & -7.48457300 & 5.21211400 \\
\hline $\mathrm{O}$ & $\mathrm{o}$ & -11.83582500 & -1.32637500 & -0.77350200 \\
\hline $\mathrm{H}$ & -1 & -9.25769100 & $-7 \cdot 30263900$ & -4.27890000 \\
\hline $\mathrm{H}$ & -1 & -4.05334000 & 2.90501500 & -0.14437700 \\
\hline $\mathrm{H}$ & -1 & -4.91371700 & 2.98079500 & -2.38539000 \\
\hline $\mathrm{H}$ & -1 & -9.26455300 & 1.09425700 & -4.27218200 \\
\hline $\mathrm{O}$ & $\mathrm{o}$ & -7.53937400 & -3.08583400 & -6.13887800 \\
\hline $\mathrm{H}$ & -1 & -5.85949000 & -4.81015900 & -6.77145100 \\
\hline $\mathrm{H}$ & -1 & -7.63561600 & -6.60953700 & 5.94181900 \\
\hline $\mathrm{H}$ & -1 & -11.06388100 & -0.67559600 & 3.43618100 \\
\hline $\mathrm{H}$ & -1 & 0.29826200 & 2.13375200 & -0.61422600 \\
\hline $\mathrm{H}$ & -1 & -1.47117400 & 2.79433400 & 0.86828900 \\
\hline $\mathrm{H}$ & -1 & 0.22680800 & 1.27611200 & 1.62542800 \\
\hline $\mathrm{H}$ & -1 & $-5 \cdot 59981300$ & -10.96122200 & 2.66496200 \\
\hline $\mathrm{H}$ & -1 & -3.36021300 & -10.88941500 & 1.80733600 \\
\hline $\mathrm{O}$ & $\mathrm{o}$ & -10.05084200 & -3.20882500 & o $\quad-5.18447700$ \\
\hline $\mathrm{H}$ & -1 & -11.75708200 & -4.80340800 & -4.27893400 \\
\hline $\mathrm{H}$ & -1 & -13.64365000 & -2.91657900 & 0.07190600 \\
\hline $\mathrm{H}$ & -1 & -13.56786700 & -0.67556100 & 0.93221600 \\
\hline $\mathrm{H}$ & -1 & -10.13959000 & -9.11347000 & 5.94186600 \\
\hline $\mathrm{H}$ & -1 & -10.99998700 & -9.18926800 & 3.70086200 \\
\hline $\mathrm{H}$ & -1 & -11.75710200 & -7.49127300 & 5.21910800 \\
\hline $\mathrm{H}$ & -1 & -9.26454800 & -1.40504100 & -6.77148900 \\
\hline $\mathrm{H}$ & -1 & 0.95626500 & 0.40105300 & -2.65003200 \\
\hline
\end{tabular}




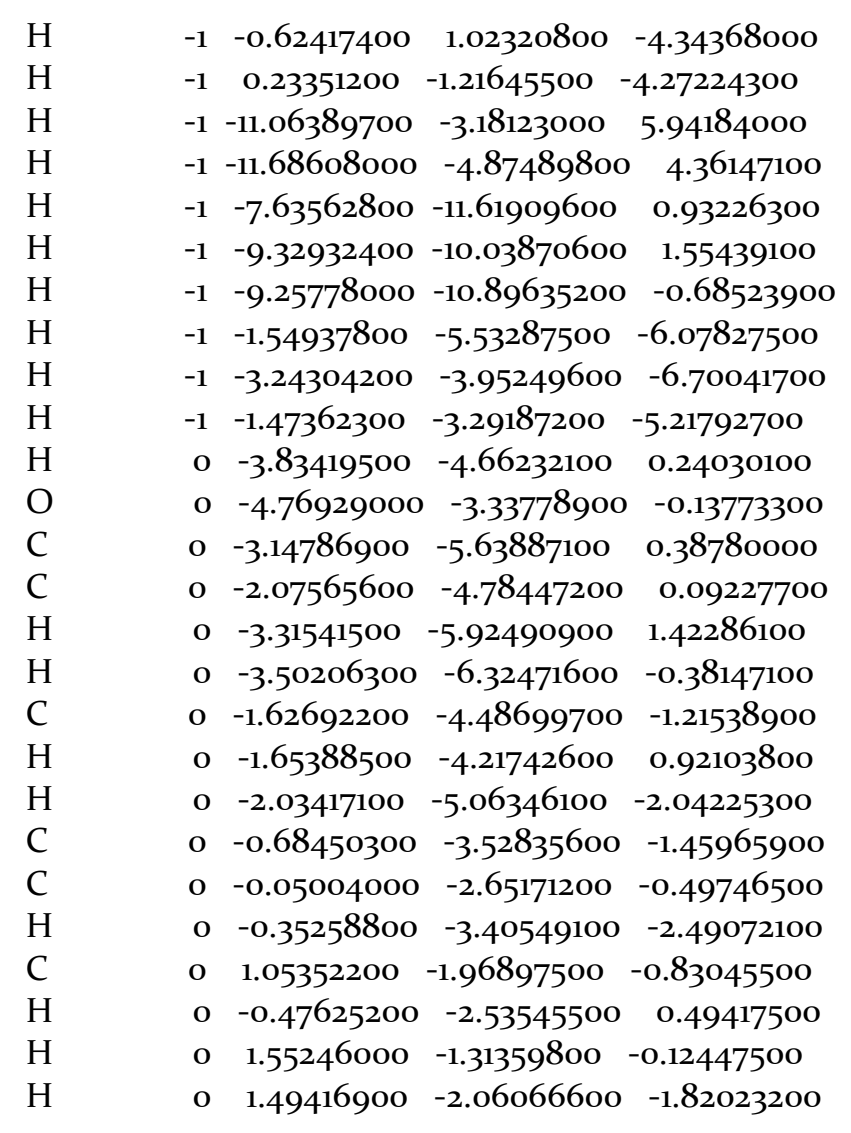

J-K* TS (H-FAU)

(Electronic Energy: -10882.8322643 Ha):

$\begin{array}{lllll}\mathrm{O} & \mathrm{o} & -5.22822600 & 2.86515300 & 3.38751000 \\ \mathrm{O} & \mathrm{o} & -0.17990400 & -5.91979800 & 1.98428200 \\ \mathrm{O} & \mathrm{o} & -0.66291200 & -1.53163900 & -0.18388300 \\ \mathrm{O} & \mathrm{o} & -6.76325600 & -0.40350600 & -1.81403600 \\ \mathrm{O} & \mathrm{o} & -3.74846300 & -5.54865500 & 3.19615100 \\ \mathrm{O} & \mathrm{o} & -5.87535000 & -0.25337100 & -4.97499800 \\ \mathrm{O} & \mathrm{o} & 0.25317000 & -1.28339200 & -4.01980400 \\ \mathrm{O} & \mathrm{o} & -2.80793100 & -2.86602200 & -1.09772400 \\ \mathrm{O} & \mathrm{o} & -5.46360800 & -2.23461100 & -3.24112100 \\ \mathrm{O} & \mathrm{o} & -1.99082100 & -2.66379800 & -4.42382600 \\ \mathrm{O} & \mathrm{o} & -5.23409100 & 3.73913300 & 0.86165600 \\ \mathrm{O} & \mathrm{o} & -7.77429100 & -4.70058200 & -3.96429600 \\ \mathrm{O} & \mathrm{o} & -2.57448200 & -6.02079400 & 0.85864600 \\ \mathrm{O} & \mathrm{o} & -5.07649900 & -8.46669000 & -0.01610900 \\ \mathrm{O} & \mathrm{o} & -3.55672200 & 0.98459800 & -4.56466600 \\ \mathrm{O} & \mathrm{o} & -0.83618000 & 0.27273800 & -2.14226900 \\ \mathrm{O} & \mathrm{o} & -4.37939900 & 0.52126300 & -1.00577200 \\ \mathrm{O} & \mathrm{o} & -0.44814800 & -3.18904000 & -2.27288700 \\ \mathrm{O} & \mathrm{o} & -5.62516000 & -2.47837900 & -0.57976500 \\ \mathrm{O} & \mathrm{o} & -4.32776200 & -2.34171800 & -5.64564900 \\ \mathrm{O} & \mathrm{o} & -2.94849300 & 3.59398800 & 2.17859500 \\ \mathrm{O} & \mathrm{o} & -7.93230400 & -5.39084200 & -1.42488400 \\ \mathrm{O} & \mathrm{o} & -5.22701600 & -6.05647100 & 1.02496300 \\ & & & & \end{array}$




\begin{tabular}{|c|c|c|c|}
\hline $\mathrm{O}$ & o $\quad-0.42497100$ & -6.58243500 & -0.59272100 \\
\hline $\mathrm{O}$ & $\begin{array}{ll}0 & -5.62604700\end{array}$ & 1.64760200 & -3 \\
\hline $\mathrm{O}$ & o $\quad-1.98430400$ & 0.80885400 & $0 \quad 0.16701700$ \\
\hline $\mathrm{O}$ & o $\quad 0.64337200$ & 0.71382100 & 0.06925600 \\
\hline $\mathrm{O}$ & o $\quad-0.78817900$ & -4.08227700 & 0.2160690 \\
\hline $\mathrm{O}$ & o $\quad-7.79882200$ & -2.78063300 & o -2.0872710 \\
\hline $\mathrm{O}$ & o $\quad-3.96236800$ & -3.74987000 & o $\quad 1.3834210$ \\
\hline $\mathrm{O}$ & o $\quad-6.60115700$ & 1.93898900 & -0.6048760 \\
\hline $\mathrm{O}$ & o $\quad-7.47415800$ & $-7 \cdot 32990800$ & 0.3642190 \\
\hline $\mathrm{O}$ & o $\quad-4.18495500$ & 1.35709300 & 1.54289100 \\
\hline $\mathrm{Si}$ & $\mathrm{o} \quad-1.23025400$ & -2.92668800 & -0.8 \\
\hline $\mathrm{Si}$ & o $\quad-6.38081600$ & -2.00209600 & -1.8927060 \\
\hline $\mathrm{Si}$ & o -3.18765100 & -6.73239200 & 4.17869800 \\
\hline $\mathrm{Si}$ & o $\quad-5.64577600$ & -1.86325700 & -4.81467500 \\
\hline $\mathrm{Al}$ & o $\quad-4.05001300$ & -2.59812600 & 0.09797300 \\
\hline $\mathrm{Si}$ & o $\quad-0.46091900$ & -2.75178000 & -3.84033600 \\
\hline $\mathrm{Si}$ & o $\quad-6.61763500$ & 3.45948200 & 0.00291100 \\
\hline Si & o $\quad-3.88435500$ & -5.32154400 & 1.57031800 \\
\hline S & o $\quad-5.18181000$ & 1.16722800 & -4.55736100 \\
\hline $\mathrm{Si}$ & $\begin{array}{ll}0 & -0.01928500\end{array}$ & 0.25960100 & -3.56041200 \\
\hline $\mathrm{S}$ & o $\quad-3.55053300$ & 0.39472200 & 0.38944100 \\
\hline S & o $\quad-4.39747900$ & 2.90091800 & 1.98636800 \\
\hline S & o $\quad-1.00300900$ & -5.64262600 & 0.59331000 \\
\hline $\mathrm{Si}$ & o $\quad-5.96330700$ & -7.50297100 & 0.95562400 \\
\hline $\mathrm{Si}$ & $\begin{array}{ll}\text { o } & -8.36392400\end{array}$ & $-4.245^{28700}$ & 03500 \\
\hline $\mathrm{Si}$ & o $\quad-0.71237400$ & 0.05310600 & -0.53 \\
\hline $\mathrm{Si}$ & o $\quad-5.84875000$ & 0.91721100 & -1.61669700 \\
\hline S & o $\quad-6.15987300$ & 3.93832900 & 4.23794900 \\
\hline S & o $\quad-0.23998700$ & -7.05183200 & 3.17747500 \\
\hline$S$ & $\begin{array}{ll}0 & -2.71\end{array}$ & -2.23480400 & 61700 \\
\hline$S$ & o $\quad-7.70048100$ & -4.06136000 & -5.47776100 \\
\hline S & $\begin{array}{ll}0 & -5.139\end{array}$ & -9.99853700 & -0.62265500 \\
\hline 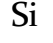 & $\begin{array}{ll}o & -2.26873600\end{array}$ & 0.80696000 & -5.55493200 \\
\hline Si & $\begin{array}{ll}0 & -2.30388800\end{array}$ & 5.05717600 & 2.59009100 \\
\hline $\mathrm{Si}$ & o $\quad-8.18814900$ & -6.97192000 & -1.07105600 \\
\hline $\mathrm{Si}$ & $0 \quad-0.17482600$ & -8.16491600 & -0.99459100 \\
\hline $\mathrm{Si}$ & o $\quad 1.37756900$ & 2.19789800 & 0.05918700 \\
\hline $\mathrm{H}$ & $-1-3.77522600$ & -8.01541500 & 3.79318900 \\
\hline $\mathrm{O}$ & o $\quad-1.55242500$ & -6.81508200 & 4.13540900 \\
\hline $\mathrm{H}$ & $\begin{array}{ll}-1 & -3.59722900\end{array}$ & -6.35626800 & 5.51717500 \\
\hline $\mathrm{O}$ & o $\quad-6.95313000$ & -2.59631900 & -5.44458900 \\
\hline $\mathrm{H}$ & $\begin{array}{ll}-1 & 0.31041600\end{array}$ & -3.70592900 & -4.61199500 \\
\hline $\mathrm{H}$ & $\begin{array}{ll}-1 & -7.76659100\end{array}$ & 3.56162900 & 0.89459400 \\
\hline $\mathrm{H}$ & $-1 \quad-6.69203600$ & 4.45220800 & -1.0599780 \\
\hline $\mathrm{H}$ & $\begin{array}{ll}-1 & -5.60693500\end{array}$ & 2.18485100 & -5.50184200 \\
\hline $\mathrm{O}$ & o $\quad-0.91172300$ & 1.03864400 & -4.67922700 \\
\hline $\mathrm{H}$ & $-1 \quad 1.24254500$ & 0.95447300 & -3.43465000 \\
\hline $\mathrm{H}$ & $\begin{array}{ll}-1 & -6.07854200\end{array}$ & -8.08373600 & $\quad 2.286678$ \\
\hline $\mathrm{H}$ & $\begin{array}{ll}-1 & -9.80561100\end{array}$ & -4.10011700 & -2.55924400 \\
\hline $\mathrm{H}$ & $\begin{array}{ll}-1 & -5.49686200\end{array}$ & 5.25419400 & 4.32034500 \\
\hline $\mathrm{H}$ & $\begin{array}{ll}-1 & -7.47708500\end{array}$ & 4.10187600 & 3.6019390 \\
\hline$\alpha$ & $\begin{array}{ll}-1 & -6.32661200\end{array}$ & 3.39190900 & $5 \cdot 58533100$ \\
\hline
\end{tabular}




$\begin{array}{lrlll}\mathrm{H} & -1 & -0.28280700 & -8.41948000 & 2.62776700 \\ \mathrm{H} & -1 & 0.94304600 & -6.88164400 & 4.00212900 \\ \mathrm{O} & \mathrm{o} & -2.28709500 & -0.69352200 & -6.20870200 \\ \mathrm{H} & -1 & -2.27078500 & -3.08588300 & -6.94560300 \\ \mathrm{H} & -1 & -6.95845900 & -5.00213800 & -6.31099200 \\ \mathrm{H} & -1 & -9.05267600 & -3.86523500 & -6.01143600 \\ \mathrm{H} & -1 & -5.13937900 & -10.97981700 & 0.47831100 \\ \mathrm{H} & -1 & -3.95813800 & -10.19570300 & -1.46023700 \\ \mathrm{H} & -1 & -6.35820800 & -10.17912600 & -1.43051500 \\ \mathrm{H} & -1 & -2.27416400 & 1.79925000 & -6.61401000 \\ \mathrm{H} & -1 & -2.77912800 & 5.45185500 & 3.92719800 \\ \mathrm{H} & -1 & -2.67055400 & 6.09911800 & 1.62011500 \\ \mathrm{H} & -1 & -0.85691100 & 4.87653300 & 2.60643800 \\ \mathrm{H} & -1 & -9.61924400 & -7.23318500 & -0.91433200 \\ \mathrm{H} & -1 & -7.63668500 & -7.83922600 & -2.12072000 \\ \mathrm{H} & -1 & 0.60163900 & -8.85668700 & 0.05750800 \\ \mathrm{H} & -1 & -1.45949100 & -8.85703800 & -1.16931100 \\ \mathrm{H} & -1 & 0.57773500 & -8.19945400 & -2.25280900 \\ \mathrm{H} & -1 & 2.73230700 & 2.02485000 & 0.59207100 \\ \mathrm{H} & -1 & 1.45448800 & 2.70645300 & -1.31997500 \\ \mathrm{H} & -1 & 0.63803800 & 3.16170300 & 0.89166300 \\ \mathrm{O} & \mathrm{o} & -3.61149100 & -1.10736500 & 0.98206800 \\ \mathrm{C} & \mathrm{o} & -0.70638000 & -2.02154400 & 2.60882000 \\ \mathrm{C} & \mathrm{o} & -1.97919000 & -2.29238500 & 2.94026300 \\ \mathrm{H} & \mathrm{o} & -0.38364200 & -1.01138400 & 2.37108000 \\ \mathrm{H} & \mathrm{o} & 0.03880800 & -2.80772100 & 2.54093900 \\ \mathrm{C} & \mathrm{o} & -2.93564700 & -1.21767400 & 2.98235400 \\ \mathrm{H} & \mathrm{o} & -2.29837500 & -3.30726600 & 3.14666800 \\ \mathrm{H} & \mathrm{o} & -2.51447900 & -0.2251100 & 2.83429800 \\ \mathrm{C} & \mathrm{o} & -4.23300100 & -1.27400400 & 3.58186800 \\ \mathrm{C} & \mathrm{o} & -4.78111500 & -2.43169300 & 4.00972200 \\ \mathrm{H} & \mathrm{o} & -4.78844500 & -0.34069100 & 3.62919800 \\ \mathrm{C} & \mathrm{o} & -6.14679900 & -2.54039600 & 4.58403300 \\ \mathrm{H} & \mathrm{o} & -4.22705000 & -3.36303900 & 3.91040800 \\ \mathrm{H} & \mathrm{o} & -6.11333400 & -3.03451700 & 5.56066100 \\ \mathrm{H} & \mathrm{o} & -6.63422000 & -1.56924200 & 4.68130000 \\ \mathrm{H} & \mathrm{o} & -6.75217800 & -3.18076200 & 3.93169600 \\ & & & & \\ & & & \end{array}$

\section{K-L* TS (H-FAU)}

(Electronic Energy: -10882.8223810 Ha):

$\begin{array}{lllll}\mathrm{O} & \mathrm{o} & -1.29207400 & 0.47302500 & -0.51206700 \\ \mathrm{O} & \mathrm{o} & -6.78141700 & -8.06541100 & 0.98838600 \\ \mathrm{O} & \mathrm{o} & -6.39843600 & -4.42130800 & -2.22858500 \\ \mathrm{O} & \mathrm{o} & -7.22363300 & 0.99461700 & 0.60720300 \\ \mathrm{O} & \mathrm{o} & -5.68456300 & -5.55522900 & 3.64945700 \\ \mathrm{O} & \mathrm{o} & -10.35646100 & 1.73472200 & -1.66564600 \\ \mathrm{O} & \mathrm{o} & -9.57534900 & -3.69663900 & -4.60920700 \\ \mathrm{O} & \mathrm{o} & -8.06058000 & -3.34629200 & -0.39904900 \\ \mathrm{O} & \mathrm{o} & -9.52972400 & -0.14268300 & 0.02173600 \\ \mathrm{O} & \mathrm{o} & -10.68181100 & -2.74775700 & -2.37673700 \\ \mathrm{O} & \mathrm{o} & -3.09065300 & 1.75389900 & -2.02283300 \\ \mathrm{O} & \mathrm{o} & -11.61610200 & 0.18936500 & 2.99398200\end{array}$




\begin{tabular}{|c|c|c|c|}
\hline $\mathrm{O}$ & o $\quad-7.89229000$ & -6.03116800 & 2.24596400 \\
\hline $\mathrm{O}$ & o $\quad-9.99977900$ & -5.28390600 & 5.07825900 \\
\hline $\mathrm{O}$ & o $\quad-8.93511800$ & 0.67602500 & -3.66376900 \\
\hline $\mathrm{O}$ & o $\quad-7.19513200$ & -2.62169000 & -4.03863000 \\
\hline $\mathrm{O}$ & o $\quad-6.10345200$ & 0.00294800 & -1.64501800 \\
\hline $\mathrm{O}$ & o $\quad-9.02777500$ & -4.90914800 & -2.29804300 \\
\hline $\mathrm{O}$ & o $\quad-7.84369500$ & -1.38679800 & 1.67587000 \\
\hline $\mathrm{O}$ & o -11.74714400 & -0.52199200 & -1.39218700 \\
\hline $\mathrm{O}$ & o $\quad-1.88278600$ & -0.39864000 & -2.96839200 \\
\hline $\mathrm{O}$ & o $\quad-9.75657600$ & -0.88330200 & 4.51123100 \\
\hline $\mathrm{O}$ & o $\quad-7.87311500$ & -4.10258100 & 4.07558200 \\
\hline $\mathrm{O}$ & o $\quad-9.34970200$ & -7.44943900 & 0.54379900 \\
\hline $\mathrm{O}$ & o $\quad-7.68286000$ & 2.10516300 & -1.79076100 \\
\hline $\mathrm{O}$ & o $\quad-4.97433300$ & -2.18701400 & -2.62493100 \\
\hline $\mathrm{O}$ & o $\quad-4.98610000$ & -4.11609100 & -4.41207300 \\
\hline $\mathrm{O}$ & o $\quad-7.41080200$ & $-5 \cdot 97168600$ & -0.44993600 \\
\hline $\mathrm{O}$ & o $\quad-9.06785200$ & 0.80589100 & 2.47943200 \\
\hline $\mathrm{O}$ & $\begin{array}{ll}\text { o } & -6.38189600\end{array}$ & -3.85222600 & 1.84434300 \\
\hline $\mathrm{O}$ & o $\quad-5.32035200$ & 2.30744500 & -0.57778000 \\
\hline $\mathrm{O}$ & o $\quad-9.18557300$ & -2.94838000 & 6.10325900 \\
\hline $\mathrm{O}$ & o $\quad-3.58896800$ & -0.67092400 & o $\quad-0.97462600$ \\
\hline $\mathrm{Si}$ & o $\quad-7.76215300$ & -4.61646400 & -1.30751000 \\
\hline $\mathrm{Si}$ & o $\quad-8.40260700$ & 0.02641500 & 1.21003500 \\
\hline $\mathrm{Si}$ & o $\quad-5.43746200$ & -6.96870800 & 4.42750200 \\
\hline $\mathrm{Si}$ & o -10.83532800 & 0.58348100 & -0.61154100 \\
\hline $\mathrm{Al}$ & o $\quad-7.01125600$ & -2.61157900 & 0.79532600 \\
\hline $\mathrm{Si}$ & o -10.17321900 & -4.10709100 & -3.13548400 \\
\hline $\mathrm{Si}$ & o $\quad-3.98236500$ & 2.92968000 & -1.28311200 \\
\hline $\mathrm{Si}$ & o $\quad-6.98029200$ & -4.83763000 & 2.93495300 \\
\hline $\mathrm{Si}$ & o $\quad-9.06293600$ & 1.95278000 & -2.64368500 \\
\hline $\mathrm{Si}$ & o $\quad-8.38121200$ & -2.73160400 & -5.16556800 \\
\hline $\mathrm{Si}$ & o $\quad-5.09283600$ & -1.23243000 & -1.28578400 \\
\hline $\mathrm{Si}$ & o $\quad-2.46768400$ & 0.29698100 & -1.62310300 \\
\hline $\mathrm{Si}$ & o $\quad-7.87752500$ & -6.84612700 & 0.83012400 \\
\hline $\mathrm{Si}$ & o $\quad-8.70319500$ & -4.36375200 & 5.45129700 \\
\hline $\mathrm{Si}$ & o -10.13601900 & 0.45048300 & 3.65496600 \\
\hline $\mathrm{Si}$ & o $\quad-5.91211600$ & -3.31698200 & -3.32458400 \\
\hline $\mathrm{Si}$ & $\begin{array}{ll}\text { o } & -6.59832400\end{array}$ & 1.34176600 & -0.84557500 \\
\hline $\mathrm{Si}$ & $0 \quad-0.05814600$ & 1.52439000 & -0.19636800 \\
\hline $\mathrm{Si}$ & o $\quad-6.34208600$ & -9.03676800 & 2.24691700 \\
\hline $\mathrm{Si}$ & o -11.71464000 & -1.51035400 & -2.68966300 \\
\hline $\mathrm{Si}$ & o -12.55803100 & 1.00043800 & 1.91835500 \\
\hline $\mathrm{Si}$ & o -11.30724600 & -5.94626100 & 5.83396100 \\
\hline $\mathrm{Si}$ & o $\quad-9.87302900$ & -0.07740700 & -4.77375600 \\
\hline $\mathrm{Si}$ & o $\quad-0.74699700$ & -0.14371700 & -4.13591600 \\
\hline $\mathrm{Si}$ & o -10.27113400 & -1.75090600 & 5.80542000 \\
\hline $\mathrm{Si}$ & o -10.49539300 & -8.42810100 & 1.21675900 \\
\hline $\mathrm{Si}$ & o $\quad-4.22542700$ & -3.76271100 & -5.84728600 \\
\hline $\mathrm{H}$ & $-1-6.45077900$ & -7.18423800 & 5.46636000 \\
\hline $\mathrm{O}$ & o $\quad-5.44312200$ & -8.20502100 & 3.33959400 \\
\hline $\mathrm{H}$ & $-1 \quad-4.11537300$ & -6.87943300 & 5.00912800 \\
\hline $\mathrm{O}$ & o -11.73390300 & 1.30437300 & 0.52949800 \\
\hline
\end{tabular}




\begin{tabular}{|c|c|c|c|}
\hline $\mathrm{H}$ & -1 & -11.29389100 & $-4.99059500 \quad-3.34513900$ \\
\hline $\mathrm{H}$ & -1 & -3.18707400 & $3.55103700-0.23123300$ \\
\hline $\mathrm{H}$ & -1 & $-4 \cdot 36497200$ & $3.90280400-2.29427100$ \\
\hline $\mathrm{H}$ & -1 & -9.29216800 & $3.16368300-3.40091900$ \\
\hline $\mathrm{O}$ & o & -8.96645200 & $-1.24414600 \quad-5.47389800$ \\
\hline $\mathrm{H}$ & -1 & -7.86218500 & $-3.29226300 \quad-6.39696600$ \\
\hline $\mathrm{H}$ & -1 & -7.87470200 & $-5.04152600 \quad 6.44673000$ \\
\hline $\mathrm{H}$ & -1 & -10.15309000 & $1.61537300 \quad 4.51366700$ \\
\hline $\mathrm{H}$ & -1 & 0.71335600 & $1.78720800 \quad-1.42637600$ \\
\hline $\mathrm{H}$ & -1 & -0.57457400 & $2.79838800 \quad 0.32962900$ \\
\hline $\mathrm{H}$ & -1 & 0.81290800 & $0.89690600 \quad 0.79931200$ \\
\hline $\mathrm{H}$ & -1 & $-7 \cdot 51282300$ & $-9.64017200 \quad 2.90532100$ \\
\hline $\mathrm{H}$ & -1 & -5.49605200 & $-10.07220300 \quad 1.67976500$ \\
\hline $\mathrm{O}$ & o & -11.18567200 & $-0.71325100-4.02626100$ \\
\hline $\mathrm{H}$ & -1 & -13.08737400 & $-1.97190700 \quad-2.94031100$ \\
\hline $\mathrm{H}$ & -1 & -13.71665700 & $0.16230700 \quad 1.65294400$ \\
\hline $\mathrm{H}$ & -1 & -12.96556000 & $2.29163700 \quad 2.47086400$ \\
\hline $\mathrm{H}$ & -1 & -10.86854100 & $-6.87989000 \quad 6.89058000$ \\
\hline $\mathrm{H}$ & -1 & -12.08283700 & $-6.67520500 \quad 4.82864500$ \\
\hline $\mathrm{H}$ & -1 & -12.14849700 & $-4.89768800 \quad 6.44031700$ \\
\hline $\mathrm{H}$ & -1 & -10.30586300 & $0.82049300 \quad-5.84558600$ \\
\hline $\mathrm{H}$ & -1 & 0.58967700 & $0.01663700-3.53113700$ \\
\hline $\mathrm{H}$ & -1 & -1.05540000 & $1.05128200-4.93683500$ \\
\hline $\mathrm{H}$ & -1 & -0.75836200 & $-1.32874700 \quad-4.99478000$ \\
\hline $\mathrm{H}$ & -1 & -10.33491200 & $-0.90089700 \quad 7.00212700$ \\
\hline $\mathrm{H}$ & -1 & -11.59792100 & $-2.34548800 \quad 5.56315700$ \\
\hline $\mathrm{H}$ & -1 & -9.90613500 & $-9.73919100 \quad 1.54809300$ \\
\hline $\mathrm{H}$ & -1 & -11.04506900 & $-7.82427900 \quad 2.43644100$ \\
\hline $\mathrm{H}$ & -1 & -11.55446800 & $-8.59950000 \quad 0.22372400$ \\
\hline $\mathrm{H}$ & -1 & $-3 \cdot 79637300$ & $-5.03770500-6.43936000$ \\
\hline $\mathrm{H}$ & -1 & -5.14241300 & $-3.08142500 \quad-6.77727600$ \\
\hline $\mathrm{H}$ & -1 & -3.04498900 & $-2.90833900 \quad-5.62145600$ \\
\hline $\mathrm{O}$ & o & $-5 \cdot 55614800$ & $-2.06912100-0.01525500$ \\
\hline $\mathrm{C}$ & o & -3.17425700 & $-5.01169600-1.76031500$ \\
\hline C & o & -4.08602100 & $-6.03152500-1.88280500$ \\
\hline C & o & -4.50047000 & $-6.70262000-0.70925900$ \\
\hline C & o & $-4 \cdot 32223600$ & $-5.98371200 \quad 0.45366400$ \\
\hline $\mathrm{H}$ & o & -3.05853400 & $-4.25882700 \quad-2.53670200$ \\
\hline $\mathrm{H}$ & $\mathrm{O}$ & -2.39455300 & $-5.04953000 \quad-1.00380300$ \\
\hline $\mathrm{H}$ & o & -4.56063900 & $-6.22896900 \quad-2.84046000$ \\
\hline $\mathrm{H}$ & o & -4.96405200 & $-7.68402700 \quad-0.71146400$ \\
\hline $\mathrm{H}$ & o & -4.38056700 & $-6.44341900 \quad 1.44042900$ \\
\hline C & o & -4.08079600 & $-4.59788400 \quad 0.35263000$ \\
\hline C & $\mathrm{O}$ & $-3 \cdot 37757800$ & $-3.78797100 \quad 1.36695400$ \\
\hline $\mathrm{H}$ & o & -4.67304900 & $-4.04121300 \quad-0.37589800$ \\
\hline $\mathrm{H}$ & o & -3.00916700 & $-2.85690400 \quad 0.93023400$ \\
\hline $\mathrm{H}$ & o & -2.59145600 & $-4.33637400 \quad 1.88749700$ \\
\hline $\mathrm{H}$ & $\mathrm{O}$ & -4.16217700 & $-3.50327500 \quad 2.08369700$ \\
\hline
\end{tabular}

\section{$\mathrm{L}-\mathrm{M}^{*} \mathrm{TS}(\mathrm{H}-\mathrm{FAU})$}

(Electronic Energy: -10882.8096717 Ha):
$\mathrm{O}$ o $\quad-1.78175900 \quad 1.21264200 \quad-0.00236900$ 
$\begin{array}{llll}\text { o } & -5.29346000 & -8.36929700 & 0.85866400\end{array}$

$\begin{array}{lllll}\mathrm{O} & 0 & -5.22401900 & -4.72573200 & -2.41493900\end{array}$

$\begin{array}{llllll}\mathrm{O} & \mathrm{O} & -8.06268600 & 0.50546000 & -0.08148500\end{array}$

$\begin{array}{lllll}\mathrm{O} & \mathrm{o} & -5.30736300 & -5.70241900 & 3.59613500\end{array}$

$\begin{array}{llllll}\mathrm{O} & \mathrm{o} & -10.37066300 & 0.35497000 & -2.49901600\end{array}$

$\begin{array}{lllll}\mathrm{O} & \mathrm{O} & -8.03265000 & -4.76997300 & -5.26464600\end{array}$

$\mathrm{O}$

$\begin{array}{llll}\text { o } & -7.31605100 & -4.01179800 & -0.88730300\end{array}$

$\begin{array}{llll}\text { o } & -9.68511700 & -1.50164500 & -0.71505400\end{array}$

$\begin{array}{llll}\text { o } & -9.71119700 & -4.11575600 & -3.29753000\end{array}$

$\begin{array}{llll}\text { o } & -3.52414200 & 2.07738500 & -1.84258400\end{array}$

$\begin{array}{llll}\text { o } & -12.17156600 & -1.53077600 & 1.71733500\end{array}$

$\begin{array}{llll}\text { o } & -7.05439600 & -6.66510200 & 1.83787700\end{array}$

$\begin{array}{llll}\text { o } & -9.71643800 & -6.48615100 & 4.24933800\end{array}$

$\begin{array}{llll}\text { o } & -8.67288500 & -0.42530900 & -4.39223700\end{array}$

$\begin{array}{llll}\text { o } & -6.12535700 & -3.13374100 & -4.37346900\end{array}$

$\begin{array}{llll}\text { o } & -6.16557400 & -0.49079500 & -1.70972800\end{array}$

$\begin{array}{llll}\text { o } & -7.64305200 & -5.79532100 & -2.82201400\end{array}$

$\begin{array}{llll}\text { o } & -7.71098300 & -1.90534700 & 1.03995800\end{array}$

$\begin{array}{llll}\text { o } & -11.37122200 & -2.13099500 & -2.66874800\end{array}$

$\begin{array}{lllll}\text { o } & -1.71093000 & 0.24949500 & -2.48801900\end{array}$

$\begin{array}{llll}0 & -10.42451500 & -2.16364500 & 3.58527900\end{array}$

$\begin{array}{lllll}\text { o } & -7.81943900 & -4.80522000 & 3.58335100\end{array}$

$\begin{array}{lllll}\text { o } & -7.80991000 & -8.38183000 & -0.03819300\end{array}$

$\begin{array}{llll}\text { o } & -7.84888900 & 1.34193400 & -2.59713300\end{array}$

$\begin{array}{lllll}\text { o } & -4.35855300 & -2.17769800 & -2.66301800\end{array}$

$\begin{array}{llll}\text { o } & -3.58996200 & -4.05651100 & -4.32814200\end{array}$

$\begin{array}{llll}\text { o } & -6.14135600 & -6.45073800 & -0.72180200\end{array}$

$\begin{array}{llll}\text { o } & -9.76691600 & -0.35079600 & 1.68675700\end{array}$

$\begin{array}{llll}\text { o } & -6.07294100 & -4.16088300 & 1.68480500\end{array}$

$\begin{array}{lllll}\text { o } & -6.01465200 & 1.99715900 & -0.79351400\end{array}$

$\begin{array}{llll}0 & -9.67321600 & -4.04047500 & 5.34406800\end{array}$

$\begin{array}{llll}0 & -3.61795100 & -0.42885600 & -0.82964900\end{array}$

$\begin{array}{llll}\text { o } & -6.62467800 & -5.21182000 & -1.67824500\end{array}$

$\begin{array}{lllll}\text { o } & -8.77320900 & -0.86074900 & 0.49922800\end{array}$

$\begin{array}{lllll}\text { o } & -4.83834300 & -7.02974300 & 4.41945400\end{array}$

o $-10.88703800 \quad-0.94678400 \quad-1.65666100$

$\begin{array}{llll}\text { o } & -6.62529500 & -3.10083500 & 0.43718900\end{array}$

$\begin{array}{llll}\text { o } & -8.76610400 & -5.31564200 & -3.89714000\end{array}$

$\begin{array}{llll}\text { o } & -4.80569100 & 2.97372700 & -1.29827000\end{array}$

$\begin{array}{lllll}\text { o } & -6.58924900 & -5.30337200 & 2.64517600\end{array}$

$\begin{array}{llll}\text { o } & -9.14123900 & 0.83616200 & -3.46171000\end{array}$

$\begin{array}{llll}\text { o } & -7.03208000 & -3.54661600 & -5.67161300\end{array}$

$\begin{array}{llll}\text { o } & -4.86456400 & -1.38713000 & -1.31194800\end{array}$

$\begin{array}{lllll}\text { o } & -2.67520600 & 0.79541100 & -1.30236600\end{array}$

$\begin{array}{llll}\text { o } & -6.59262300 & -7.44232000 & 0.47338000\end{array}$

$\begin{array}{lllll}\text { o } & -8.76330400 & -5.28661900 & 4.81176200\end{array}$

$\begin{array}{lllll}\text { o } & -10.94344300 & -0.94437700 & 2.63509000\end{array}$

$\begin{array}{llll}\text { o } & -4.85214100 & -3.52758600 & -3.44500000\end{array}$

$\begin{array}{lllll}\text { o } & -7.03148200 & 0.82663700 & -1.28342900\end{array}$

$\begin{array}{llll}\text { o } & -0.91573700 & 2.54040700 & 0.47449700\end{array}$

$\begin{array}{llll}\text { o } & -4.84069300 & -9.23117800 & 2.18384400\end{array}$

$\begin{array}{llll}\text { o } & -10.92283400 & -3.16109700 & -3.85202400\end{array}$ 


\begin{tabular}{|c|c|c|c|}
\hline $\mathrm{Si}$ & o -13.1006690o & -0.96790100 & 0.48372600 \\
\hline $\mathrm{Si}$ & o -10.93364300 & -7.45130000 & 4.79474000 \\
\hline $\mathrm{Si}$ & o -9.16027200 & -1.35540800 & -5.64457000 \\
\hline $\mathrm{Si}$ & o -0.50343000 & 0.78825200 & -3.47328500 \\
\hline $\mathrm{Si}$ & o -10.93186300 & -3.13309100 & 4.80732900 \\
\hline $\mathrm{Si}$ & o $\quad-8.77811600$ & -9.62159600 & 0.45929100 \\
\hline $\mathrm{Si}$ & $\begin{array}{ll}0 & -2.69027300\end{array}$ & -3.54570700 & -5.61636400 \\
\hline $\mathrm{H}$ & $\begin{array}{ll}-1 & -5.92889300\end{array}$ & -7.48692600 & 5.28071800 \\
\hline $\mathrm{O}$ & o $\quad-4.36689700$ & o $\quad-8.22247200$ & 3.39190300 \\
\hline $\mathrm{H}$ & $\begin{array}{ll}-1 & -3.68929800\end{array}$ & -6.62924200 & 5.20881600 \\
\hline $\mathrm{O}$ & o -12.16097800 & -0.46554100 & -0.76935800 \\
\hline $\mathrm{H}$ & $-1 \quad-9.58685200$ & -6.44734600 & -4.28222400 \\
\hline $\mathrm{H}$ & $\begin{array}{ll}-1 & -4.38241400\end{array}$ & 3.76036700 & -0.14768800 \\
\hline $\mathrm{H}$ & $\begin{array}{ll}-1 & -5.24279800\end{array}$ & 3.83614200 & -2.38870300 \\
\hline $\mathrm{H}$ & $\begin{array}{ll}-1 & -9.59359300\end{array}$ & 1.94956200 & -4.27547600 \\
\hline $\mathrm{O}$ & o $\quad-7.88654200$ & -2.24351000 & -6.14770300 \\
\hline $\mathrm{H}$ & $-1 \quad-6.18852000$ & -3.95479300 & -6.77478600 \\
\hline $\mathrm{H}$ & $\begin{array}{ll}-1 & -7.96469300\end{array}$ & -5.75420900 & 5.93853100 \\
\hline $\mathrm{H}$ & $-1-11.39295900$ & 0.17975200 & 3.43287300 \\
\hline $\mathrm{H}$ & $\begin{array}{ll}-1 & -0.03079100\end{array}$ & 2.98911300 & -0.61753900 \\
\hline $\mathrm{H}$ & $-1 \quad-1.80023900$ & 3.64970900 & 0.86499200 \\
\hline $\mathrm{H}$ & $-1 \quad-0.10225400$ & 2.13146100 & 1.62212700 \\
\hline $\mathrm{H}$ & $-1 \quad-5.92888600$ & -10.10591500 & 2.66167900 \\
\hline $\mathrm{H}$ & $-1 \quad-3.68928500$ & -10.03408300 & 1.80403400 \\
\hline $\mathrm{O}$ & o -10.38792900 & o $\quad-2.32232000$ & -5.15959500 \\
\hline $\mathrm{H}$ & $-1-12.08613900$ & -3.94807700 & -4.28223800 \\
\hline $\mathrm{H}$ & $-1-13.97271000$ & -2.06124000 & 0.06859500 \\
\hline $\mathrm{H}$ & $\begin{array}{ll}-1 & -13.89692400\end{array}$ & 0.17977600 & 0.92890300 \\
\hline $\mathrm{H}$ & $-1-10.46866100$ & -8.25813500 & 5.93855800 \\
\hline $\mathrm{H}$ & $-1-11.32905600$ & -8.33393000 & 3.69755200 \\
\hline $\mathrm{H}$ & $-1-12.08617500$ & -6.63593500 & 5.21580000 \\
\hline $\mathrm{H}$ & $-1 \quad-9.59360700$ & -0.54970800 & -6.77479900 \\
\hline $\mathrm{H}$ & $\begin{array}{ll}-1 & 0.62720900\end{array}$ & 1.25640500 & -2.65333600 \\
\hline $\mathrm{H}$ & $-1-0.95322600$ & 1.87855500 & $-4 \cdot 34700700$ \\
\hline $\mathrm{H}$ & $-1 \quad-0.09554800$ & -0.36111700 & -4.27554600 \\
\hline $\mathrm{H}$ & $-1-11.39297200$ & -2.32588900 & 5.93853500 \\
\hline $\mathrm{H}$ & $-1-12.01514600$ & -4.01955800 & 4.35816100 \\
\hline $\mathrm{H}$ & $\begin{array}{ll}-1 & -7.96470300\end{array}$ & -10.76377100 & 0.92896400 \\
\hline $\mathrm{H}$ & $-1 \quad-9.65841200$ & -9.18337600 & 1.55109300 \\
\hline $\mathrm{H}$ & $\begin{array}{ll}-1 & -9.58685300\end{array}$ & -10.04102000 & -0.68855000 \\
\hline $\mathrm{H}$ & $-1 \quad-1.87844300$ & -4.67754500 & -6.08164400 \\
\hline $\mathrm{H}$ & $-1 \quad-3.57211800$ & -3.09711900 & -6.70377200 \\
\hline $\mathrm{H}$ & $-1 \quad-1.80266700$ & -2.43653100 & -5.22125700 \\
\hline $\mathrm{O}$ & o $\quad-5.10603600$ & -2.39505900 & -0.10517400 \\
\hline $\mathrm{C}$ & o $\quad-3.34364600$ & -5.33131400 & 1.02173600 \\
\hline $\mathrm{C}$ & o $\quad-3.07004800$ & -5.23377200 & -0.27745700 \\
\hline $\mathrm{C}$ & o $\quad-2.47793200$ & -3.89911700 & -0.61333900 \\
\hline $\mathrm{C}$ & o $\quad-3.08800600$ & -4.05235500 & 1.77358700 \\
\hline $\mathrm{H}$ & o $\quad-3.75461300$ & -6.20815100 & 1.51056500 \\
\hline $\mathrm{H}$ & o $\quad-3.27378200$ & -5.97359100 & -1.04055600 \\
\hline $\mathrm{H}$ & o $\quad-2.80461400$ & -3.34363200 & -1.48663500 \\
\hline $\mathrm{H}$ & o $\quad-2.45572$ & -4.16881400 & 2.65878100 \\
\hline
\end{tabular}




$$
\begin{array}{lllll}
\mathrm{H} & \mathrm{o} & -4.04425600 & -3.61911500 & 2.08463700 \\
\mathrm{C} & \mathrm{o} & -2.49971900 & -3.11353300 & 0.71742300 \\
\mathrm{H} & \mathrm{o} & -2.79553200 & -2.07113900 & 0.59854000 \\
\mathrm{C} & \mathrm{o} & -1.10509000 & -3.63650400 & -0.17634200 \\
\mathrm{H} & \mathrm{o} & -0.54944900 & -2.82538900 & -0.64021200 \\
\mathrm{H} & \mathrm{o} & -1.34645000 & -2.95284700 & 1.02190100 \\
\mathrm{H} & \mathrm{o} & -0.54681100 & -4.44077800 & 0.29656800
\end{array}
$$

\section{M-P* TS (H-FAU)}

(Electronic Energy: -10882.8543587 Ha):
$\mathrm{O}$
$\begin{array}{llll}0 & -1.45690600 & 0.35254100 & 0.00049600\end{array}$
$\mathrm{O}$
$\begin{array}{llll}\text { o } & -4.97465500 & -9.20967300 & 0.88117900\end{array}$
$\mathrm{O}$
$\begin{array}{llll}\text { o } & -4.88889200 & -5.61184600 & -2.42490500\end{array}$
$\begin{array}{lllll}\mathrm{O} & \mathrm{o} & -7.73637000 & -0.31987400 & -0.06329600\end{array}$
$\begin{array}{lllll}\mathrm{O} & \mathrm{o} & -4.99387500 & -6.57610800 & 3.60614100\end{array}$
$\begin{array}{llllll}\mathrm{O} & \mathrm{o} & -10.03446200 & -0.49487100 & -2.48739900\end{array}$
$\begin{array}{lllll}\mathrm{O} & \mathrm{o} & -7.68809100 & -5.62919200 & -5.24579000\end{array}$
$\begin{array}{lllll}\mathrm{O} & \mathrm{O} & -6.92858800 & -4.80789600 & -0.90815200\end{array}$
$\begin{array}{lllll}\mathrm{O} & \mathrm{o} & -9.33968400 & -2.34629000 & -0.70588300\end{array}$
$\begin{array}{lllll}\mathrm{O} & \mathrm{o} & -9.38515400 & -4.96774800 & -3.29504800\end{array}$
$\begin{array}{lllll}\mathrm{O} & \mathrm{o} & -3.13601300 & 1.29017900 & -1.86243700\end{array}$
$\begin{array}{lllll}\mathrm{O} & \mathrm{o} & -11.84021300 & -2.38685700 & 1.72128600\end{array}$
$\begin{array}{lllll}\mathrm{O} & \mathrm{o} & -6.75598900 & -7.50411500 & 1.84203700\end{array}$
$\begin{array}{llllll}\mathrm{O} & \mathrm{o} & -9.38346200 & -7.33629600 & 4.24828700\end{array}$
$\begin{array}{lllll}\mathrm{O} & \mathrm{o} & -8.34750400 & -1.28197200 & -4.38839700\end{array}$
$\begin{array}{lllll}\mathrm{O} & \mathrm{o} & -5.77549800 & -3.99430800 & -4.36777500\end{array}$
$\begin{array}{lllll}\mathrm{O} & \mathrm{O} & -5.90610900 & -1.42950300 & -1.66045900\end{array}$
$\begin{array}{lllll}\mathrm{O} & \mathrm{o} & -7.33819400 & -6.64447500 & -2.79084300\end{array}$
$\begin{array}{lllll}\mathrm{O} & \mathrm{O} & -7.35668100 & -2.73061900 & 1.04867100\end{array}$
O $\quad 0 \quad-11.03117300 \quad-2.98241600 \quad-2.66041500$
$\begin{array}{llllll}\mathrm{O} & \mathrm{O} & -1.38713700 & -0.60828500 & -2.49137400\end{array}$
$\begin{array}{lllll}\mathrm{O} & \mathrm{o} & -10.09431600 & -3.02083800 & 3.58920500\end{array}$
$\begin{array}{lllll}\mathrm{O} & \mathrm{o} & -7.48617500 & -5.63607000 & 3.57966500\end{array}$
$\begin{array}{lllll}\mathrm{O} & \mathrm{o} & -7.48337700 & -9.23318400 & -0.03602700\end{array}$
$\begin{array}{llllll}\mathrm{O} & \mathrm{o} & -7.50670600 & 0.47149600 & -2.59564700\end{array}$
$\begin{array}{lllll}\mathrm{O} & \mathrm{o} & -4.04278500 & -3.05368100 & -2.62916400\end{array}$
$\begin{array}{lllll}\mathrm{O} & \mathrm{O} & -3.24264500 & -4.91278500 & -4.31686700\end{array}$
$\begin{array}{lllll}\mathrm{O} & \mathrm{o} & -5.82871600 & -7.27474100 & -0.68886800\end{array}$
$\begin{array}{lllll}\mathrm{O} & \mathrm{o} & -9.43312900 & -1.20613800 & 1.69959500\end{array}$
$\begin{array}{lllll}\mathrm{O} & \mathrm{O} & -5.70034300 & -5.03113200 & 1.67382300\end{array}$
$\begin{array}{lllll}\mathrm{O} & 0 & -5.62337100 & 1.08071400 & -0.82922200\end{array}$
$\begin{array}{lllll}\mathrm{O} & \mathrm{o} & -9.34055800 & -4.89417300 & 5.34575700\end{array}$
$\begin{array}{llllll}\mathrm{O} & 0 & -3.35963900 & -1.19337800 & -0.85955900\end{array}$
$\begin{array}{llllll}\mathrm{Si} & 0 & -6.29606800 & -6.05901200 & -1.67726000\end{array}$
$\begin{array}{llllll}\text { Si } & & 0 & -8.43666500 & -1.69662900 & 0.50994100\end{array}$
$\begin{array}{lllll}\mathrm{Si} & 0 & -4.50477900 & -7.89774200 & 4.42808000\end{array}$
$\begin{array}{lllll}\mathrm{Si} & 0 & -10.54862500 & -1.79892200 & -1.64706100\end{array}$
$\begin{array}{lllll}\mathrm{Al} & 0 & -6.29283100 & -3.94779900 & 0.46865400\end{array}$
Si $\quad \begin{array}{lllll}\text { A } & \text { o } & -8.44179800 & -6.16980800 & -3.88939200\end{array}$
$\begin{array}{llllll}\mathrm{Si} & 0 & -4.45545700 & 2.12208600 & -1.30560000\end{array}$
$\begin{array}{lllll}\mathrm{Si} & 0 & -6.25518500 & -6.15601800 & 2.64939100\end{array}$
$\begin{array}{lllll}\mathrm{Si} & 0 & -8.80916600 & -0.01955700 & -3.45799000\end{array}$ 


\begin{tabular}{|c|c|c|c|c|}
\hline $\mathrm{Si}$ & O & -6.69261200 & -4.40347800 & -5.66009700 \\
\hline $\mathrm{Si}$ & o & -4.53429600 & -2.23104600 & -1.31178100 \\
\hline $\mathrm{Si}$ & $\mathrm{O}$ & -2.34168100 & -0.02424000 & -1.32005500 \\
\hline $\mathrm{Si}$ & $\mathrm{O}$ & -6.27398100 & -8.29212600 & 0.49114800 \\
\hline $\mathrm{S} 1$ & o & -8.42710500 & -6.13697600 & 4.80923500 \\
\hline $\mathrm{Si}$ & o & -10.61487900 & -1.80127300 & 2.64249200 \\
\hline $\mathrm{Si}$ & $\mathrm{O}$ & -4.50716400 & $-4 \cdot 39849500$ & -3.43441200 \\
\hline $\mathrm{Si}$ & $\mathrm{O}$ & -6.70191300 & -0.04923900 & -1.27723800 \\
\hline $\mathrm{Si}$ & $\mathrm{O}$ & -0.58512500 & 1.68460600 & 0.47838800 \\
\hline $\mathrm{Si}$ & $\mathrm{O}$ & -4.50957000 & -10.08633300 & 2.19437000 \\
\hline $\mathrm{Si}$ & $\mathrm{O}$ & -10.59607800 & -4.01248600 & -3.84898600 \\
\hline $\mathrm{Si}$ & o & -12.77090300 & -1.82377900 & 0.48880300 \\
\hline $\mathrm{Si}$ & $\mathrm{O}$ & -10.60081700 & -8.30388300 & $4 \cdot 79653700$ \\
\hline $\mathrm{Si}$ & $\mathrm{O}$ & -8.83182300 & -2.21275700 & -5.64144900 \\
\hline $\mathrm{Si}$ & $\mathrm{O}$ & -0.17039000 & -0.06775900 & -3.47217000 \\
\hline $\mathrm{Si}$ & $\mathrm{O}$ & -10.60201000 & -3.99029100 & 4.81266900 \\
\hline $\mathrm{Si}$ & o & -8.44884300 & -10.47692700 & 0.46250200 \\
\hline $\mathrm{Si}$ & o & -2.35599500 & -4.40035500 & -5.61525000 \\
\hline $\mathrm{H}$ & -1 & $-5 \cdot 59982700$ & -8.34224400 & 5.28400400 \\
\hline $\mathrm{O}$ & o & -4.03421600 & -9.10128200 & $3 \cdot 41784700$ \\
\hline $\mathrm{H}$ & -1 & $-3 \cdot 36023200$ & $-7 \cdot 48457100$ & 5.21211100 \\
\hline $\mathrm{O}$ & o & -11.82334100 & -1.32264800 & -0.75960400 \\
\hline $\mathrm{H}$ & -1 & -9.25769100 & $-7 \cdot 30264600$ & -4.27890500 \\
\hline $\mathrm{H}$ & -1 & -4.05333600 & 2.90501900 & -0.14437300 \\
\hline $\mathrm{H}$ & -1 & -4.91372100 & 2.98079900 & -2.38539300 \\
\hline $\mathrm{H}$ & -1 & -9.26455900 & 1.09425800 & -4.27218100 \\
\hline $\mathrm{O}$ & o & -7.55470600 & -3.10308200 & -6.13073900 \\
\hline $\mathrm{H}$ & -1 & -5.85950500 & -4.81015300 & -6.77145500 \\
\hline $\mathrm{H}$ & -1 & -7.63561600 & -6.60953600 & $5 \cdot 94181900$ \\
\hline $\mathrm{H}$ & -1 & -11.06388000 & -0.67559700 & 3.43618000 \\
\hline $\mathrm{H}$ & -1 & 0.29826200 & 2.13375400 & -0.61422900 \\
\hline $\mathrm{H}$ & -1 & -1.47117500 & 2.79433600 & 0.86829200 \\
\hline $\mathrm{H}$ & -1 & 0.22681000 & 1.27611000 & 1.62542900 \\
\hline $\mathrm{H}$ & -1 & $-5 \cdot 59981500$ & -10.96122800 & 2.66496300 \\
\hline $\mathrm{H}$ & -1 & $-3 \cdot 36021400$ & -10.88941100 & 1.80733400 \\
\hline $\mathrm{O}$ & o & -10.06329700 & -3.17607200 & -5.15842400 \\
\hline $\mathrm{H}$ & -1 & -11.75706800 & -4.80339200 & -4.27893500 \\
\hline $\mathrm{H}$ & -1 & -13.64365000 & -2.91657800 & 0.07190600 \\
\hline $\mathrm{H}$ & -1 & -13.56786600 & -0.67556300 & 0.93221300 \\
\hline $\mathrm{H}$ & -1 & -10.13959000 & -9.11347100 & $5 \cdot 94186600$ \\
\hline $\mathrm{H}$ & -1 & -10.99 & -9.18926800 & o 3.70086200 \\
\hline $\mathrm{H}$ & -1 & -11.75710300 & -7.49127200 & 5.21910800 \\
\hline $\mathrm{H}$ & -1 & -9.26454200 & -1.40506000 & -6.77147800 \\
\hline $\mathrm{H}$ & -1 & 0.95626500 & 0.40105300 & -2.65003100 \\
\hline $\mathrm{H}$ & -1 & -0.62417400 & 1.02320800 & $-4 \cdot 34368000$ \\
\hline $\mathrm{H}$ & -1 & 0.23351300 & -1.21645600 & -4.27224300 \\
\hline $\mathrm{H}$ & -1 & -11.06389800 & -3.18122900 & $5 \cdot 94184100$ \\
\hline $\mathrm{H}$ & -1 & -11.68607900 & -4.87489900 & $4 \cdot 36147100$ \\
\hline $\mathrm{H}$ & -1 & -7.63562700 & -11.61909400 & 0.93226300 \\
\hline $\mathrm{H}$ & -1 & -9.32932400 & -10.03870700 & 1.55439200 \\
\hline $\mathrm{H}$ & -1 & -9.25778200 & -10.89635400 & -0.68524100 \\
\hline $\mathrm{H}$ & -1 & -1.54937600 & $-5 \cdot 53287900$ & -6.07827800 \\
\hline
\end{tabular}




$\begin{array}{lllll}\mathrm{H} & -1 & -3.24304300 & -3.95249500 & -6.70041900 \\ \mathrm{H} & -1 & -1.47362200 & -3.29187000 & -5.21792700 \\ \mathrm{H} & \mathrm{o} & -3.58175500 & -3.38781300 & 0.88169500 \\ \mathrm{O} & \mathrm{o} & -4.72620500 & -3.21516500 & -0.03496100 \\ \mathrm{C} & \mathrm{o} & -2.55841600 & -3.76253800 & 1.54950700 \\ \mathrm{C} & \mathrm{o} & -2.05660700 & -5.07411300 & -0.88968000 \\ \mathrm{C} & \mathrm{o} & -1.52432200 & -3.83982000 & -0.68947000 \\ \mathrm{C} & \mathrm{o} & -1.79838300 & -3.16444500 & 0.54163800 \\ \mathrm{H} & \mathrm{o} & -2.63232500 & -3.23572300 & 2.49833300 \\ \mathrm{H} & \mathrm{o} & -2.00296200 & -5.51894200 & -1.88307500 \\ \mathrm{H} & \mathrm{o} & -1.03748600 & -3.29823700 & -1.49316000 \\ \mathrm{H} & \mathrm{o} & -1.54164400 & -2.10883400 & 0.62346100 \\ \mathrm{C} & \mathrm{o} & -2.81651700 & -5.81861200 & 0.15410000 \\ \mathrm{H} & \mathrm{o} & -3.87766700 & -5.72750300 & -0.11984800 \\ \mathrm{H} & \mathrm{o} & -2.60224700 & -6.88969700 & 0.08305700 \\ \mathrm{C} & \mathrm{o} & -2.58755300 & -5.28283100 & 1.56579600 \\ \mathrm{H} & \mathrm{o} & -1.62087600 & -5.63297800 & 1.95077200 \\ \mathrm{H} & \mathrm{o} & -3.37089600 & -5.63625700 & 2.23475800\end{array}$

